DEPARTAMENTO DE AUTOMÁTICA, INGENIERÍA ELÉCTRICA Y

\author{
ELECTRÓNICA E INFORMÁTICA INDUSTRIAL
}

UNIVERSIDAD POLITÉCNICA DE MADRID

ESCUELA TÉCNICA SUPERIOR DE INGENIEROS INDUSTRIALES

\title{
Large-signal, Black-box Modelling of Bidirectional Battery Chargers
}

\section{TESIS DOCTORAL}

\author{
Autor: Antreas Naziris \\ Máster en Ingeniería Eléctrica y Electrónica, Newcastle University \\ Director: Javier Uceda Antolín
}

Doctor Ingeniero Industrial por la Universidad Politécnica de Madrid 



\section{Acknowledgements}

As a five-year journey comes to an end, I would like to thank the people who made it easier to cope with.

Firstly, my advisor Javier Uceda for his invaluable help and patience guiding me through this path until the end which without him this thesis would not be possible. "Eu そnv"

A special thanks is given to Airan Frances for his priceless help whenever was needed through all this time.

Also, I would like to thank the people in DC systems, Energy conversion \& Storage department at Delft University for the amazing time I had during my internship.

The time through PhD would not be the same without its people, especially to Branislav, Diego, Guillermo, LiXin, Yann, Hesam, David, Vladan, LiCheng. Thank you for the memories and I hope we keep in touch.

Finally, a tremendous gratitude to my family for supporting me in these 5 years path. I would like to thank my uncle Xópns for his support in my weak moments and remind me why I started it in the first place. My

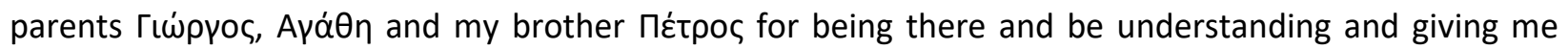
courage to continue. This thesis is dedicated to my mother who taught me that nothing is unreachable once we do the first step. "Znv" 



\section{Abstract}

In a world of constantly evolving and with the green sector of energy blooming, in our days is not strange more and more companies and governmental agencies investing in this area. Based on this sector is dedicated the idea of this thesis. An area which is currently evolving and is based among others on the electric vehicles sector and together with them the battery chargers. Electric vehicle is a perfect example of which the transformation from the old oil era will pass to the new electricity era. The traditional conventional car is giving up its place for the hybrid and the second to the full electric. Currently in the market all three types coexist.

In the near future, full battery chargers are expected be released in the market in a larger amount that exist today. The variety of the battery charger schemes have a large spectrum already and by evolving to become more efficient, subsequently the number of schemes is increasing. Therefor a unified method must present in order to fast identify the behaviour of this amount of chargers fast, efficient and also avoid creating any problems interfacing the grid at system level.

As it is well known the battery chargers in their core are AC/DC converters. The proposed solution in this document to deal with this problem is the black-box model. Black-box models can determine the dynamic behaviour of power electronic converters by analysing the output results created by the changes in the input variables. The black-box approach is already used in an adequate extent in DC/DC converters for DC microgrids and in $D C / A C$ for $A C$ microgrids. Although in the AC/DC section the studies are limited due to the complexity of the system and that is the main contribution of this thesis.

In this research black-box modelling techniques used in DC/DC previously are extended to single-phase and three-phase $A C / D C$ converters and more precisely to battery chargers. Firstly, the model is transformed from $A C / D C$ to $D C / D C$ model with a Park transformation and then by using the input/output data is identified in a small signal model. In a small signal model, the validity is limited to small perturbation around the operating point applied in the input variables of the converter. The validity of this model has been expressed with an electrical equivalent circuit and mathematically through their transfer functions and it is considered as one of the main contributions of this thesis.

The linear model approach is validated by both virtual and experimental tests. In the virtual part, with the aid of PSIM simulator, an AC/DC converter is created. This converter model, it is assumed to be an accurate representation of an actual AC/DC converter and therefor having its dynamic behaviour compared with the results of the black-box models. The results obtained by the virtual and experimental tests, show the accuracy of dynamic behaviour representation of single and three-phase battery chargers in the smallsignal spectrum of operation. 
Following the small-signal operation, large perturbations applied to the input variables of the converter and a clear loss of accuracy observed leading to a large-signal black-box approach in case of large perturbations. This approach name is polytopic and it is already used in DC/DC and DC/AC converters. In this thesis it will be used for first time in a two-stage single and three phase bidirectional AC/DC converter like battery charger.

Polytopic model approach consists of a group of different linear models in different operating points combined with nonlinear weighting functions which are depended on the instantaneous input variables to create a single nonlinear structure. Accuracy in the selection of the local model together with the weighting functions are essential for the accuracy of the system. Keeping this in mind and looking back in the bibliography it can be assumed that Double Sigmoid is the most suitable weighting function for the AC/DC battery charger application. This large-signal nonlinear models represent another main contribution of the thesis.

In the same manner as in small-signal, large-signal nonlinear approach experimentally. On the experimental part, tests proceeded to both power flow direction tests in V2G and G2V operating modes. The results coming out from the large-signal tests show with no surprise better accuracy than in smallsignal models in case of large perturbations as input variables. This conclusion validates in case of large perturbation operation, the correct use of the large-signal black-box models for the dynamic behaviour representation in single and three-phase of two-stage bidirectional battery chargers. 


\section{Resumen}

En un mundo en constante evolución y con el sector verde de la energía floreciendo, en nuestros días no es extraño que cada vez haya más empresas y administraciones invirtiendo en esta área. A este sector se dedica la idea de esta tesis. Un ámbito que está evolucionando en la actualidad y que se basa, entre otros en la proliferación de los vehículos eléctricos y junto a ellos los imprescindibles cargadores de baterías. El vehículo eléctrico es un ejemplo perfecto de que la transformación de la antigua era del petróleo pasará a la nueva era de la electricidad. El coche convencional tradicional está cediendo su lugar al híbrido y el éste, en un siguiente paso, al totalmente eléctrico. Actualmente en el mercado conviven los tres tipos.

En un futuro próximo, se espera que la demanda de cargadores de batería crezca continuamente y se lancen al mercado en una cantidad mucho mayor que la existente en la actualidad. La variedad de esquemas de cargadores de batería es, hoy en día, bastante grande y, su evolución hacia sistemas más eficientes supondrá la aparición de un número, aún mayor, de soluciones diferentes. Por lo tanto, debe existir un método unificado para identificar el comportamiento de todos estos cargadores de baterías, en particular en su interacción con la red, para estimar sus efectos y evitar problemas desde la perspectiva del sistema eléctrico.

Como es bien sabido, los cargadores de batería son esencialmente convertidores AC/DC. La solución propuesta en esta tesis para abordar este problema es el modelo de caja negra. Los modelos de caja negra pueden determinar el comportamiento dinámico de los convertidores electrónicos de potencia analizando los resultados de salida creados por las perturbaciones en las variables de entrada. El enfoque de caja negra ya se utiliza de manera adecuada en convertidores $\mathrm{CC} / \mathrm{CC}$ para microrredes de CC y en CC/CA para microrredes de CA. Los estudios previos de convertidores CA/CC en caja negra son limitados debido a la complejidad del sistema, por lo que su estudio en profundidad es el principal aporte de esta tesis.

En esta investigación, las técnicas de modelado de caja negra utilizadas anteriormente en CC/CC se extienden a convertidores $\mathrm{CA} / \mathrm{CC}$ monofásicos y trifásicos y, más precisamente, a cargadores de baterías. En primer lugar, el modelo se transforma de CA/CC a CC/CC mediante la transformada de Park y luego, utilizando los datos de entrada/salida, se identifica un modelo de pequeña señal. En los modelos de pequeña señal su validez está limitada a pequeñas perturbaciones en las entradas del convertidor alrededor del punto de operación. La validez de este modelo se ha expresado con un circuito eléctrico equivalente y matemáticamente mediante las funciones de transferencia, siendo ésta una de las principales aportaciones de esta tesis. 
El enfoque del modelo lineal está validado por pruebas virtuales y experimentales. En la parte virtual, con la ayuda del simulador PSIM, se crea un convertidor CA/CC. Se supone que este modelo de convertidor es una representación precisa de un convertidor $\mathrm{CA} / \mathrm{CC}$ real y, por lo tanto, su comportamiento dinámico se compara con los resultados de los modelos de caja negra. Los resultados obtenidos por las pruebas virtuales y experimentales muestran la precisión de la representación del comportamiento dinámico de cargadores de baterías monofásicos y trifásicos en las condiciones de operación de pequeña señal.

Después de la operación de pequeña señal, se aplicaron grandes perturbaciones a las variables de entrada del convertidor y se observó una clara pérdida de precisión que condujo a un enfoque de caja negra de gran señal en caso de grandes perturbaciones. La técnica empleada se basa en los modelos politópicos, que ya se emplea en convertidores CC/DC y CC/CA. En esta tesis se utilizará por primera vez en un convertidor $\mathrm{CA} / \mathrm{CC}$ bidireccional monofásico y trifásico de dos etapas como cargador de batería.

Los modelos politópicos están compuestos de un grupo de diferentes modelos lineales en diferentes puntos de operación, combinados con funciones de ponderación no lineales que dependen de los valores instantáneos de las variables de entrada para crear una única estructura no lineal. La precisión en la selección del modelo local junto con las funciones de ponderación es esencial para la precisión del sistema. Teniendo esto en cuenta y mirando hacia atrás en la bibliografía, se puede concluir que Double Sigmoid es la función de ponderación más adecuada para modelos de cargador de baterías CA/CC. Estos modelos no lineales de gran señal representan otra de las contribuciones de esta tesis.

De la misma manera que en los modelos en caja negra de pequeña señal, los modelos en caja negra de gran señal, se han probado experimentalmente. En la parte experimental, las pruebas se realizaron para convertidores bidireccionales en ambos modos de funcionamiento: V2G y G2V. Los resultados de las pruebas en gran señal muestran, sin sorpresa, una mayor precisión que en los modelos de pequeña señal en caso de grandes perturbaciones en las variables de entrada. Esta conclusión valida, en caso de grandes perturbaciones de las variables de entrada, los modelos politópicos en caja negra, en gran señal, para la representación del comportamiento dinámico de cargadores de batería monofásicos y trifásicos de dos etapas, unidireccionales y bidireccionales. 
Contents

Acknowledgments

Abstract

iii

Resumen

v

Contents

vii

List of Figures

$\mathbf{x}$

List of Tables

xiv

List of Acronyms

XV

1 Introduction $\quad 1$

$1.1 \mathrm{EV}$ technology . . . . . . . . . . . . . . . . . . . . . . . . . . . . . 1

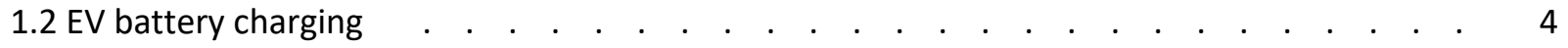

1.3 Smart charging . . . . . . . . . . . . . . . . . . . . . . . . . . . . . 6

1.4 Modelling battery chargers

1.5 Contributions . . . . . . . . . . . . . . . . . . . . . . . . . . . . . . . . 7

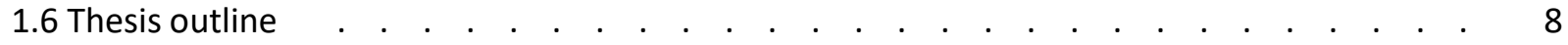


2 Modelling Battery Chargers $\quad 10$

2.1 Introduction in battery chargers $\quad . \quad$. . . . . . . . . . . . . . . . . . . . 10

2.1.1 On and off board chargers

2.1.2 Single and three-phase battery chargers . . . . . . . . . . . . . . . . 13

2.1.3 Unidirectional and Bidirectional structure $\quad . \quad$. . . . . . . . . . . . . . . . . . 13

2.2 Modelling Requirements . . . . . . . . . . . . . . . . . . . . . . . . . . 14

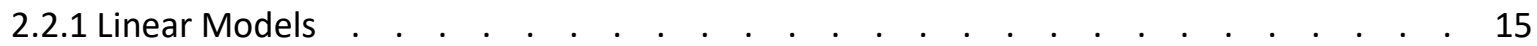

2.2.2 Small-Signal Black-box Behavioural Model . . . . . . . . . . . . . . . . . . . 16

Modelling procedure . . . . . . . . . . . . . . . . . . . . . . . 20

2.2.2.1 Parametric estimation methods $\quad$. . . . . . . . . . . . . . . . . . . . . 22

[A] Linear system identification $\quad$. . . . . . . . . . . . . . . . . . . . . 22

2.2.3 Nonlinear system identification . . . . . . . . . . . . . . . . . . . . . . 24

2.2.4 Large-signal Black-box Behavioural Model . . . . . . . . . . . . . . . . . . 26

Modelling Procedure . . . . . . . . . . . . . . . . . . . . . . . . . . . . 28

2.3 Conclusions . . . . . . . . . . . . . . . . . . . . . . . . . . . . . . . 28

3 Simulation results $\quad 31$

3.1 Introduction . . . . . . . . . . . . . . . . . . . . . . . . . . . . . . . . . . . . . 31

3.2 Boost rectifier . . . . . . . . . . . . . . . . . . . . . . . . . . . . 32

3.2.1 Boost Rectifier Controller . . . . . . . . . . . . . . . . . . . . . . . . . . . . . 34

3.3 DC-DC converter . . . . . . . . . . . . . . . . . . . . . . . . . . . . . . . . . . . . . . 41

3.3.1 DC-DC converter controller . . . . . . . . . . . . . . . . . . . . . . 45

3.4 Battery . . . . . . . . . . . . . . . . . . . . . . . . . . . . . . . . . . . . 47 
3.4.1 Charging Technique . . . . . . . . . . . . . . . . . . . . . . . . . . . . 48

3.4.2 Soft charging . . . . . . . . . . . . . . . . . . . . . . . . . . . . . 51

3.5 Black-box model for linear simulation of a battery charger . . . . . . . . . . . . 51

3.5.1 Comparison of black-box and switching model . . . . . . . . . . . . . . . . . 54

3.6 Introduction of system level battery chargers $\quad . \quad$. . . . . . . . . . . . . . . . . . . 63

3.7 Small-signal Black-box model for linear simulation of a system level battery chargers . . . . 64

3.7.1 Comparison of small-signal black-box and switching model . . . . . . . . . . . . 68

$\begin{array}{ll}4 & \text { Experimental results }\end{array}$

4.1 Introduction . . . . . . . . . . . . . . . . . . . . . . . . . . . . . . . . . . 72

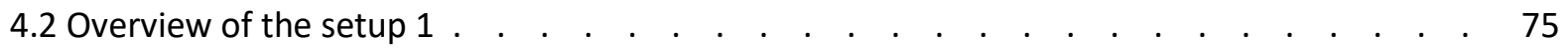

4.3 Black-box model for experimental setup 1 . . . . . . . . . . . . . . . . . . . . . . . 77

4.3.1 Comparison of small-signal black-box model and experimental setup 1 . . . . . . 80

4.3.2 Non-linear polytopic model $\quad$. . . . . . . . . . . . . . . . . . . . . . . . 82

4.3.3 Comparison of black-box polytopic model with experimental setup $1 \quad$. . . . . . . . 84

4.4 Experimental result in the setup 2 with Li-ion batteries $\quad . \quad$. . . . . . . . . . . . . . . $\quad$. 88

4.4.1 Black-box model for experimental setup 2 of a battery charger with Lithium-Ion batteries90 4.4.2 Comparison of black-box model and experimental setup 2 . . . . . . . . . . . . . . 91

5 Conclusions and future work $\quad 95$

5.1 Introduction

5.2 Conclusions . . . . . . . . . . . . . . . . . . . . . . . . . . . . . . . . . . . . . . 97

5.2.1 Contributions . . . . . . . . . . . . . . . . . . . . . . . . . . . . . . . 98

5.3 Future work . . . . . . . . . . . . . . . . . . . . . . . . . . . . . . . . 99

$\begin{array}{ll}\text { Bibliography } & 101\end{array}$ 


\section{List of Figures}

1.1 Electric Vehicle types in market . . . . . . . . . . . . . . . . . . . . . . . . 2

1.2 Electric Vehicle conductive types for battery charging $\quad$. . . . . . . . . . . . . . . . . . . 5

1.3 Daily Electricity price with and without the usage of $\mathrm{EV}$ in $\mathrm{V} 2 \mathrm{G} \bmod$. . . . . . . . . . . . . . 6

2.1 Galvanic isolation between two circuit parts . . . . . . . . . . . . . . . . . . . 11

2.2 Charging of an EV system with both on and off board charging $\quad . \quad$. $\quad . \quad$. . . . . . . . . . 12

2.3 Unidirectional and bidirectional EV's interacting in a microgrid . . . . . . . . . . . . . . . 14

2.4 White, grey, and black-box model concepts . . . . . . . . . . . . . . . . . . . . . . . 17

2.5 Battery charger transformation to D-Q framework: a) 3ph AC/DC battery charger transformation to D$Q$ framework, b) 1 ph AC/DC battery charger transformation to D-Q framework . . . . . . . . . . . 18

2.6 Block diagram of the black-box model $\quad . \quad$. . . . . . . . . . . . . . . . . . . . . . . . 18

2.7 Equivalent circuit of the black-box model $\quad . \quad$. . . . . . . . . . . . . . . . . . . . . . . . . 19

2.8 Three phase AC input voltage in the D-Q framework: (a) Operating point, (b) Step in the d-component,

(c) Step in the q-component $\quad . \quad$. . . . . . . . . . . . . . . . . . . . . . . . . . . . . 21

2.9 Up-left: ARX model structure, Up-right: ARMAX model structure, Down-left: OE model structure, Down right: BJ model structure $\quad$. . . . . . . . . . . . . . . . . . . . . . . . . . . . . . . . 22

2.10 Up: Wiener model structure, Down: Hammerstein model structure . . . . . . . . . . . . . 24

2.11 Up: Wiener-Hammerstein model structure, Down: Hammerstein-Wiener model structure . . 24

2.12 Polytopic model structure $\quad$. . . . . . . . . . . . . . . . . . . . . . . . . . . . . . . 25

2.13 Detailed structure of polytopic model structure . . . . . . . . . . . . . . . . . . . . . $\quad 27$

2.14 Simplified Polytopic model . . . . . . . . . . . . . . . . . . . . . . . . . . . . . . . 27

3.1 Battery charger's block diagram . . . . . . . . . . . . . . . . . . . . . . . 32 
$3.2 \mathrm{AC}$ and DC Voltage across MOSFET's full bridge $\quad . \quad$. . . . . . . . . . . . . . . . . . . . 33

3.3 Complete boost rectifier circuit . . . . . . . . . . . . . . . . . . . . . . . . . . . 33

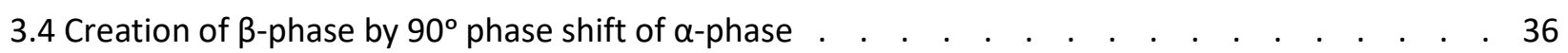

3.5 Digital form of APF controller . . . . . . . . . . . . . . . . . . . . . . . . . . . . 37

$3.6 \Theta$ angle obtained by phase locked loop $\quad . \quad$.

3.7 Full scheme of boost rectifier controller . . . . . . . . . . . . . . . . . . . . . . . . . . 38

3.8 Block diagram of the internal current regulator $\quad . \quad$. . . . . . . . . . . . . . . . . . . . 39

3.9 Block diagram of the voltage regulator $\quad . \quad$. . . . . . . . . . . . . . . . . . . . . . . . 40

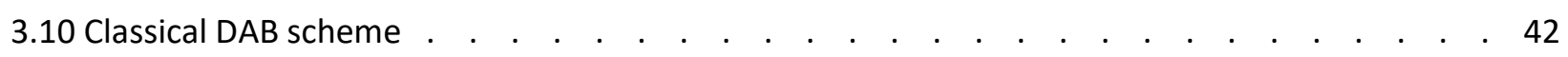

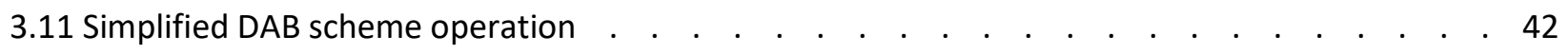

3.12 Obtaining phase shifting between the two parts of DAB $\quad . \quad$. . . . . . . . . . . . . . . . 43

3.13 Full scheme of DC-DC converter . . . . . . . . . . . . . . . . . . . . . . . . . . . 44

3.14 Current controller block diagram . . . . . . . . . . . . . . . . . . . . . . . . . . 46

3.15 Voltage controller block diagram . . . . . . . . . . . . . . . . . . . . . . . . . . . . . . . 46

3.16 Battery cell circuit $\quad . \quad$. . . . . . . . . . . . . . . . . . . . . . . . . . . . . . . 47

3.17 EV battery charging technique using CC/CV technique $\quad . \quad$. . . . . . . . . . . . . . . . . . . $\quad$. 49

3.18 Battery circuit scheme . . . . . . . . . . . . . . . . . . . . . . . . . . 50

3.19 Controller of battery circuit . . . . . . . . . . . . . . . . . . . . . . . . . . . . . 50

3.20 Switching single-phase bidirectional battery charger . . . . . . . . . . . . . . . . . . . 51

3.21 Block diagram for a black-box model of battery charger . . . . . . . . . . . . . . . . 52

3.22 Identified transfer functions for battery charger . . . . . . . . . . . . . . . . . . . . 53

3.23 Equivalent circuit of the black-box model of battery charger $\quad . \quad$. . . . . . . . . . . . . 54

3.24 Results comparison between switching and black-box model for a step of 1KVar . . . . . . . 57 
3.25 Results comparison between switching and black-box model for a step of $60 \mathrm{~V}$. . . . . . . 58

3.26 Results comparison between switching and black-box model for a step of 6A $\quad$. . . . . . . . 59

3.27 Results comparison between switching and black-box model for a step of $-3 A$. . . . . . . 60

3.28 Results comparison between switching and black-box model for a step of $-6 \mathrm{~A} \quad$. . . . . . . 61

3.29 Block diagram of system level battery chargers $\quad . \quad$. . . . . . . . . . . . . . . . . . . 63

3.30 System level of 2 connected battery chargers $\quad$. . . . . . . . . . . . . . . . . . . . . . . 64

3.31 Block Diagram of the battery charger with two batteries . . . . . . . . . . . . . 65

3.32 Block diagram of the black-box model showing the perturbation of the input and output variables .

3.33 Results comparison between switching and black-box model for DC output of primary DC-DC converter . . . . . . . . . . . . . . . . . . . . . . . . . . . . . . . . 69

3.34 Results comparison between switching and black-box model for DC output of secondary DC-DC converter . . . . . . . . . . . . . . . . . . . . . . . . . . . . . . . 69

3.35 Results comparison between switching and black-box model for AC output of Current . . . . . 70

3.36 Results comparison between switching and black-box model for DC bus voltage connection point .

4.1 CINERGIA B2C30 independent unipolar mode dc outputs with 3-phase power supply . . . . . 73

4.2 CINERGIA B2C30 equipment back-to-back topology $\quad . \quad$. . . . . . . . . . . . . . . . . . 73

4.3 Open CINERGIA B2C30 cabinet displaying different features of the equipment $\quad$. . . . . . . . 74

4.4 CINERGIA B2C30 human machine interface control . . . . . . . . . . . . . . . . . . . . 74

4.5 Grid to Vehicle mode connection . . . . . . . . . . . . . . . . . . . . . . . 76

4.6 Vehicle to Grid mode connection . . . . . . . . . . . . . . . . . . . . . . . . . 76

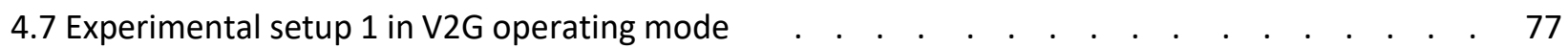

4.8 Block diagram for the small-signal black-box model for the experimental setup 1 . . . . . . 78 
4.9 Identified transfer functions for the small-signal model in experimental setup $1 \quad$. . . . . . 79

4.10 Equivalent circuit of the small-signal black-box model of experimental setup 1 . . . . . . . 79

4.11 Results comparison between switching and black-box models for AC current response (upper) with $\mathrm{d}$-q components in a $1 \mathrm{~A}$ step test in G2V mode . . . . . . . . . . . . . . . . . . . . . . . 81

4.12 Results comparison between switching and small-signal black-box model method for AC current

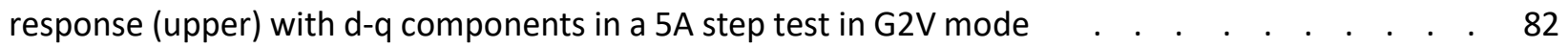

4.13 Polytopic nonlinear approach . . . . . . . . . . . . . . . . . . . . . . 82

4.14 Polytopic model example of transfer and weighting functions combination . . . . . . . . $\quad$. 83

4.15 Block diagram of double sigmoid weighting functions $\quad$. $\quad$. . . . . . . . . . . . . . . . . . 84

4.16 Summation of linear models recreating a bend for a nonlinear model (left), Real life application of polytopic model (right) . . . . . . . . . . . . . . . . . . . . . . . . . . . . . 84

4.17 Results comparison between switching and polytopic black-box model method for AC current response (upper) with $d$-q components in a 5A step test in G2V mode . . . . . . . . . . . . . . . $\quad$. 86

4.18 Results comparison between switching and polytopic black-box model method for AC current

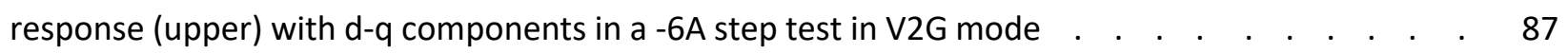

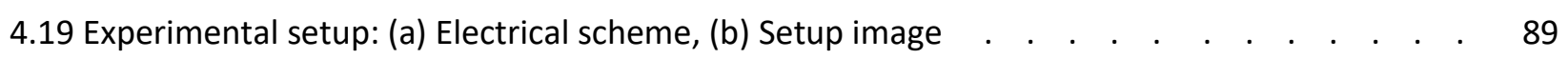

4.20 Experimental setup with Li-ion batteries and input/output points of data collection . . . . . 90

4.21 Results comparison between switching and black-box model for three AC current phases along with $\mathrm{d}$-q components and the DC battery current for a $2 \mathrm{~A}$ in a G2V mode test . . . . . . . . . . . . . . . 92

4.22 Results comparison between switching and black-box model for three AC current phases along with $\mathrm{d}$-q components and the DC battery current for a +3A step in a V2G mode test . . . . . . . . . . 93 


\section{List of Tables}

3.1 Boost rectifier parameter values . . . . . . . . . . . . . . . . . . . . . . 33

3.2 DC-DC converter parameter values. . . . . . . . . . . . . . . . . . . . . . . . . . . . 44

3.3 Identified transfer functions from the switching model . . . . . . . . . . . . . . . 62

3.4 Identified transfer functions from the system level model . . . . . . . . . . . . . . . 68

4.1 Identified transfer functions from the small-signal model in experimental setup 1 . . . . . $\quad$. 80

4.2 Identified transfer functions for G2V operation in z-domain $\quad . \quad$. . . . . . . . . . . . . 85

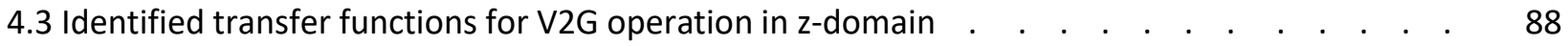

4.4 Main characteristics of the components and instruments in the experimental setup $\quad . \quad . \quad . \quad$. 89

4.5 Identified transfer functions from the system level model experimental setup with Li-ion batteries 91 


\section{List of Acronyms}

AC Alternative Current

Aexp Ampere exponential

APF All-Pass Filter

Armax Auto Regressive moving Average with eXternal model input

ARX Auto Regressive with eXternal model input (ARX)

BEV Battery Electric Vehicle

BMS Battery Management System

CAN Controller Area Network

CC Constant Current

CCS-combo Combined Charging System combo

Chademo CHArge de MOve

CNG Cinergia

CO2 Carbon Dioxide

CV Constant Voltage

DAB Dual Active Bridge

DC Direct Current

DSO Distributor System Operator

E-bike Electric bicycle

EV Electric Vehicle

FCEV Fuel Cell Electric Vehicle

FEV Full Electric Vehicle 
FPE Final Prediction Error

G2V Grid to Vehicle

HEV Hybrid Electric Vehicle

ICE Internal Combustion Engine

IGBT Insulated-Gate Bipolar Transistor

KG Kilogram

KM Kilometre

KW Kilowatt

Li-Ion Lithium Ion

LTI Linear Time Invariant

MOSFET Metal Oxide Semiconductor Field Effect Transistor

MPPT Maximum Power Point Tracking

N Neutral

OCV Open Circuit Voltage

OE Output Error

PCC Point of Common Coupling

PE Protective Earth terminal

PFC Power Factor Corrector

PHEV Plugin Hybrid Electric Vehicle

PI Proportional Integral

PLL Phase Locked Loop

PSIM Power simulation

PWM Pulse Width Modulation 
soc State Of Charge

TF Transfer Function

THD Total Harmonic Distortion

TSO Transmission system operator

V2G Vehicle to Grid

WF Weighting Function 


\title{
CHAPTER
}

\author{
1 Introduction
}

\subsection{EV technology}

As we advance in the information age, the scars left by the traditional industry become more and more evident, particularly the ones left to the environment. These scars come from the extended use of conventional energy sources like coal or oil. Some might say that this was necessary in order to advance in technology and that nothing comes without a "price". The "price of this evolution came in the form of different consequences to the environment like greenhouse gases emissions, ozone layer depletion, a rise of the temperature, and more [1]. Since the degradation of the environment is getting worse, some measures need to be taken.

During the last decades, non-conventional power sources have been introduced to the energy market. Some of these are the renewable or inexhaustible sources that do not cause environmental pollution [2]. The Kyoto Protocol [3] and Paris Agreement are international treaties, which set certain rules to stop further degradation. In the Paris Agreement date packages (2020, 2030 and 2050) it was agreed to make the reversal of environmental destruction more realistic [4]. Thanks to that, in the last years, a respectable share of the power sources has turned to renewable source. A quite recent but fast evolving example of initiatives attenuating environmental degradation is the electric vehicle (EV) technology.

Until now, the main energy source for vehicles has been fossil fuel (Gasoline and Diesel). By igniting the fuel into an internal combustion engine (ICE) the energy is harvested. This ignition causes an explosion driving down a cylinder and producing a rotation motion, like pushing the pedal of a bicycle. This is a smart way of producing mechanical force, but it has many flaws. One of those is the complex and expensive engine with a significant amount of precise moving parts. Also, the heat of the engine wastes $50 \%$ of the energy produced in a normal car, showing a very low efficiency. This heat loss and the exhaust gases 
emitted in the rate of $1 \mathrm{~L}$ of gasoline to $2,3 \mathrm{~kg}$ of $\mathrm{CO} 2$ give the final push for an alternative path of energy [5].

EV technology goes back to the last quarter of the 20th century but lately has come back and looks very promising. Rechargeable batteries, which are charged from electrical energy sources (wind turbines, solar panels...), can provide power to an electrical drivetrain to move a vehicle. This can be a definition of an electric vehicle. Opposed to the ICE engine, the electric motor operates in a cleaner manner. Due to rejection and attraction of a magnetic field, a coil attached to the rotor starts to rotate. One of the advantages of the electric motor over the ICE engine is the lower maintenance costs due to the simpler structure and operating mode.

Also, the energy efficiency of electrical motors is much higher and offers the opportunity to take back energy to the battery by regenerative braking. Regenerative breaking or regeneration is the action of storing energy back into the battery while using the break and use it when it is needed. This energy is lost in a conventional car. The energy efficiency of an $\mathrm{EV}$ in comparison with a conventional vehicle is noticed
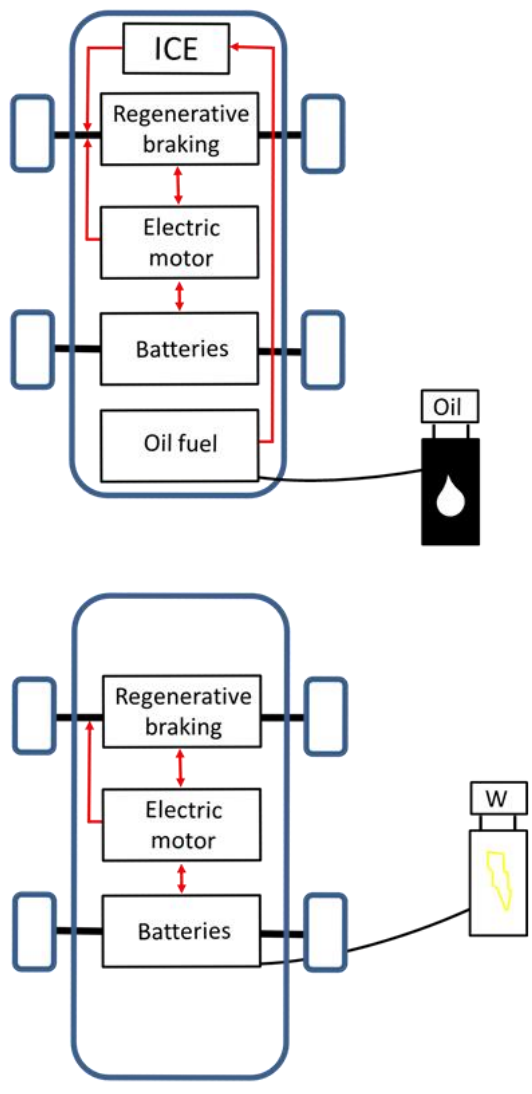
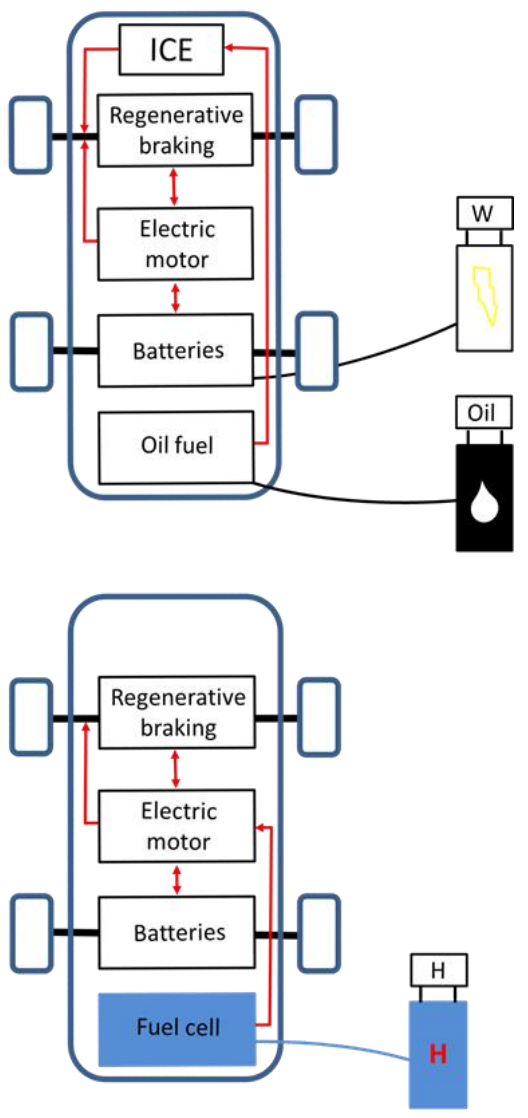

Figure 1.1 Electric Vehicle types in market 
in a certain degree with a normal car and even more with a sport car. Taking into consideration all these advantages, the question which rises is, why not turn to EV technology already?

Fossil fuel car engines (gasoline and diesel) might have a lot of disadvantages which are resolved by the electric vehicles but in the case of energy storage it still has the upper hand. At the beginning of the new millennium (2000) lead acid batteries were 350 times worse - in terms of energy density Whr/Kg- in storage than a gasoline vehicle, with $3000 \mathrm{~kg}$ batteries for a $500 \mathrm{~km}$ autonomy. Recently, in 2015 , Lithium Ion batteries have dropped the batteries storage burden to $400 \mathrm{~kg}$ per $500 \mathrm{~km}$ reducing to 50 times worse than a gasoline vehicle. Other unsolved issues are the recharging time, battery lifetime, etc.

The idea of using a technology based on EV -in extent- can give a new breath to our already bruised environment. Only the advantage of not using conventional power source as oil could be enough reason to give it a chance. The amount of $\mathrm{CO} 2$ reduction by changing the fuel of only one application would be massive. In addition, electricity as a fuel is cheaper, where taxes on $\mathrm{CO} 2$ emissions play a crucial role $[6,7,8]$ and the maintenance of the vehicles can be faster and significantly reduced $[9,10]$. Different kind of EV's, like Hybrid EVs (HEV), Plugin Hybrid EVs (PHEV), Fuel Cell EVs (FCEV) and Full EV (FEV) [11, 12], already exist on the market (Figure 1.1).

Hybrid EV is a mix of an electric vehicle and a conventional vehicle and the next step from a conventional vehicle. The combination of hybrid EV operation is of only combustion engine usage, both combustion engine and electrical engine and only electrical engine, depending on the circumstances. Regenerative braking is the only mechanism to charge the battery [13]. Therefore, the fuel saving can reach up to $20 \%$ compared to HEV, the Plug-in HEV can be charged by plugging it into an outlet. PHEV can also operate with both conventional fuel and alternative electrical energy, but with the ability of a bigger range of electricity usage due to the extra power plug. The disadvantage which a PHEV has over a full EV is the cost of the drive train (both HEV and PHEV have similar power train but with HEV not having external charging infrastructure) and the maintenance due to the two different charging features. Second disadvantage is the $\mathrm{CO} 2$ emissions caused in gasoline operation of the vehicle (this disadvantage also applies and on HEV).

Putting aside the conventional fuel, the Full EV run exclusively on electricity. As it was mentioned before, the advantages of this vehicle are many from the cheap energy source, low maintenance, small and efficient motor, to the green operation. The main disadvantage is the energy storage, due to the size of the battery and the limited recharging times. A problem which is currently resolved with the FEV's becoming cheaper and more accessible to the middle class. Moreover, another disadvantage is the charging speed of the FEV for $250 \mathrm{~km}$ trip, that needs 1 hour to charge $50 \mathrm{kw}$ in comparison with gasoline that just takes a few minutes [14].

A relatively new kind of EV is the Fuel Cell EV. The FCEV operates entirely with compressed liquid hydrogen. The fuel cell interacts with the hydrogen and with an installed electric motor which drive the wheels. This procedure of refilling the tank takes only some minutes and even if the battery is relatively small it lasts for a long range. The main drawback is the efficiency, which is lower than the FEV due to the big amount of energy that is lost from the conversion hydrogen-electricity and back with $1 / 3$ of the energy 
dissipating. Also, price development of fuel cells, storage and electrolysis are major factors for the future of the FCEV's.

\subsection{EV battery charging}

There are three common methods for charging EVs. The conductive charging which can be classified in $A C$ and DC charging; the inductive charging and the battery swapping technology [15]. The first two have the charger placed either inside (onboard) or outside (offboard) the vehicle. In AC conductive charging an onboard charger is used while an offboard AC/DC converter is used for the DC conductive chargers. In case of the inductive method a combination of both chargers is used. In the battery swapping method, the batteries are removed and replaced by a new one already charged.

The most common charging method is the conductive. AC charging, [16] as it was mentioned, uses an onboard charger allowing a connection to the grid inexpensively. The voltage and frequency of the AC power varies depending on the country and on the number of phases (single or three-phase). Due to the absence of registration for a global $A C$ connector, the different $A C$ power characteristics depending on the countries and the large variety of EVs on the market, the AC connectors are globally generalised in four types. USA and Japan share the same type of converter (type 1), while currently in Europe there are two (type 2 and 3). China, on the other hand, has its own AC connector. Although, in Europe the type 3 is almost dominated by the type 2 and China's connector is similar to type 2. Therefore, the main types of AC connectors are the type 1 and 2 .

The type 1 is used exclusively for single phase charging having two live phases (one of them used as neutral), a protective earth cable and two signal pins for charging control from the pilot controller and the connectivity confirmation between the charging point and the vehicle. The power rates of this type are $120 \mathrm{~V}$ with maximum $16 \mathrm{~A}$ and $240 \mathrm{~V}$ with maximum $80 \mathrm{~A}$. The type 2 connector can be used for single phase, three-phase, and for DC charging. The connector is composed of $3 \mathrm{AC}$ phases $(\mathrm{L1}, \mathrm{L} 2, \mathrm{~L} 3)$ a protective earth terminal (PE) and a neutral $(\mathrm{N})$. Two signal pins for charging control and connectivity are also present. The power rates for this type are $230 \mathrm{~V}$ with maximum $80 \mathrm{~A}$ for the single phase and $400 \mathrm{~V}$ with maximum $63 \mathrm{~A}$ for the three-phase charging.

DC charging [17] in comparison with AC is significantly more expensive, however it provides higher power and faster charging. The higher power and the increase of charging speed lead to larger chargers, which is the reason to use offboard chargers. The DC charging, following the AC paradigm, is divided in five connectors. The first two types of connectors are the CCS-combo 1 and CCS-combo 2 used in USA and Europe, respectively. Both connectors pin configurations are similar to the AC connector types previously mentioned. The CCS-combo 1 has the same pins as the AC type 1 connector with the difference of two DC power pins for fast charging. Likewise, the CCS-combo 2 consists of the same pins as AC type 2 connector, with two DC power pins for fast charging. The power rate of those two chargers can reach a current of $350 \mathrm{~A}$ with a DC voltage varying from 200 to $1000 \mathrm{~V}$. 

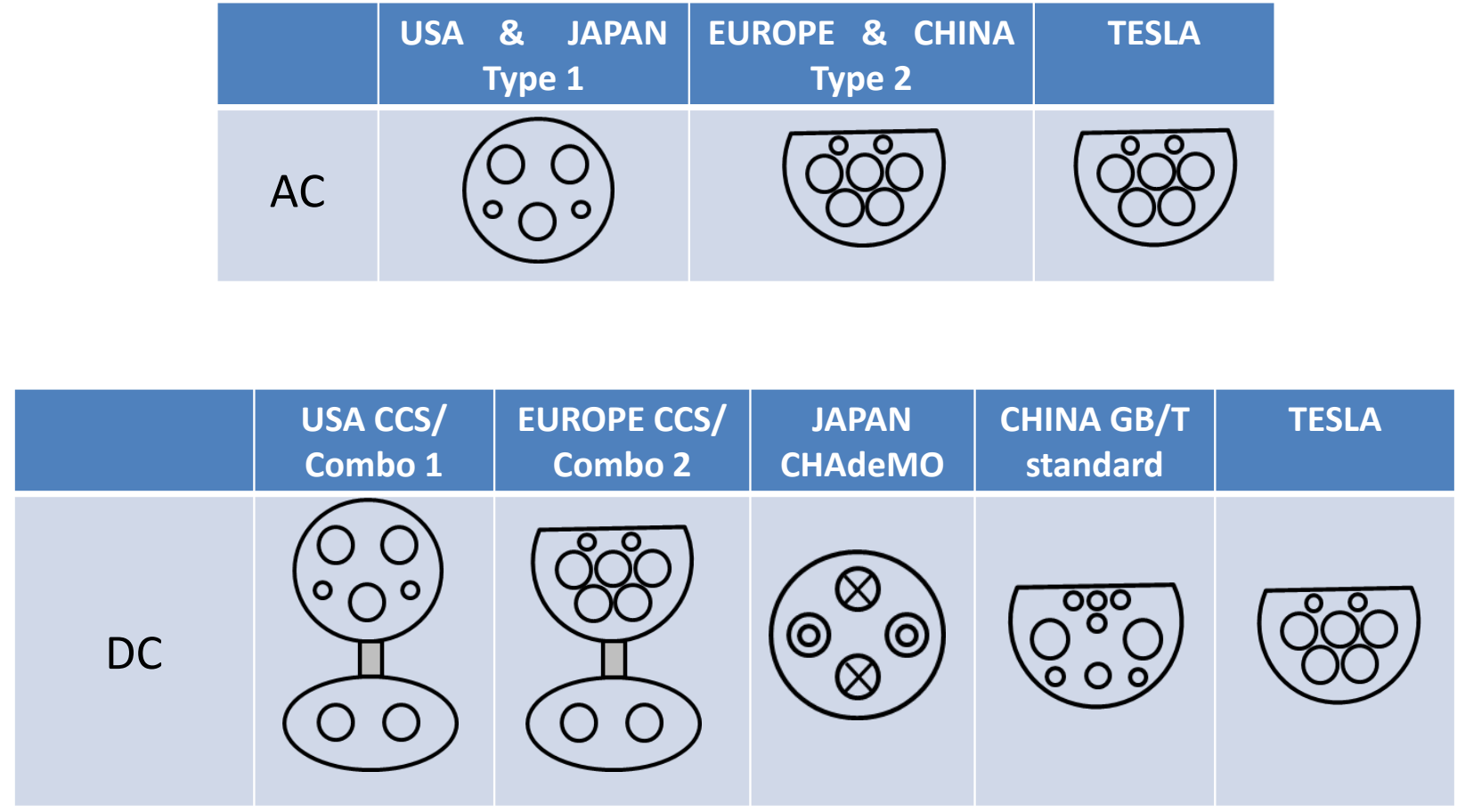

Figure 1.2 Electric Vehicle conductive types for battery charging

The third type of DC charger, named Chademo, is used exclusively for Japan made vehicles. This connector has three power pins and uses the Controller Area Network (CAN) protocol to communicate with the 6 signal pins. The power using this connector can reach up to $500 \mathrm{~V}$ and $400 \mathrm{~A}$. The fourth type of DC connector is the TESLA connector. As it can be observed in the figure 1.2 the AC connectors has the same pin configuration with type 2 . Although the difference with the AC type 2 connector is that the TESLA can use the same connector for $D C$ charging and $A C$ but only on single phase. Therefore, the pin configuration for $A C$ is consisted of two power, two signal and one earth pin. In DC, the TESLA plug orientation consists of two DC power couples (positive and negative pins), the two signal (control and proximity) and one earth pins. The rating for TESLA in AC is $240 \mathrm{~V}$ single phase with maximum $17.2 \mathrm{Kw}$ and in DC $400 \mathrm{~V}$ with maximum $300 \mathrm{~A}$ for DC power. Lastly, with the GB/T standard, there is the Chinese DC connector. The pin configuration of this charger consists of five power and four signal pins. The power pins are distributed as follows: two for DC power, two for auxiliary low voltage power and one for earth. The signals have proximity pilot and for CAN communication from 2 each [17]. The current of this charger can reach 250A with different DC voltages from $750 \mathrm{~V}$ to $1000 \mathrm{~V}$. 


\subsection{Smart charging}

The charging of an EV could be applied in an efficient manner [18] with a lower cost and an economic profit for the owner of the vehicle (Figure 1.3). This method can be achieved through intelligent procedures by a controlled charging called smart charging. The charging schedule can be controlled in order to avoid problems in the grid. Firstly, a reduction of the demand peak in the network can be achieved by shifting the high peak times for charging. Also becoming more sustainable by using renewable sources to charge the vehicle (hypothetically existing in the network). Finally, the charging time can take place at times when the electricity is cheaper, and this energy can be sold back to the grid whenever the price is higher with a financial gain.

By taking advantage of the EV battery for a Vehicle to Grid (V2G) operation, an extra power supply system acting as a renewable source reduces the peak demand and consequently reduces the emissions. Although, some disadvantages can arise like the faster degrading of the battery since the charging cycles of the battery are increased.

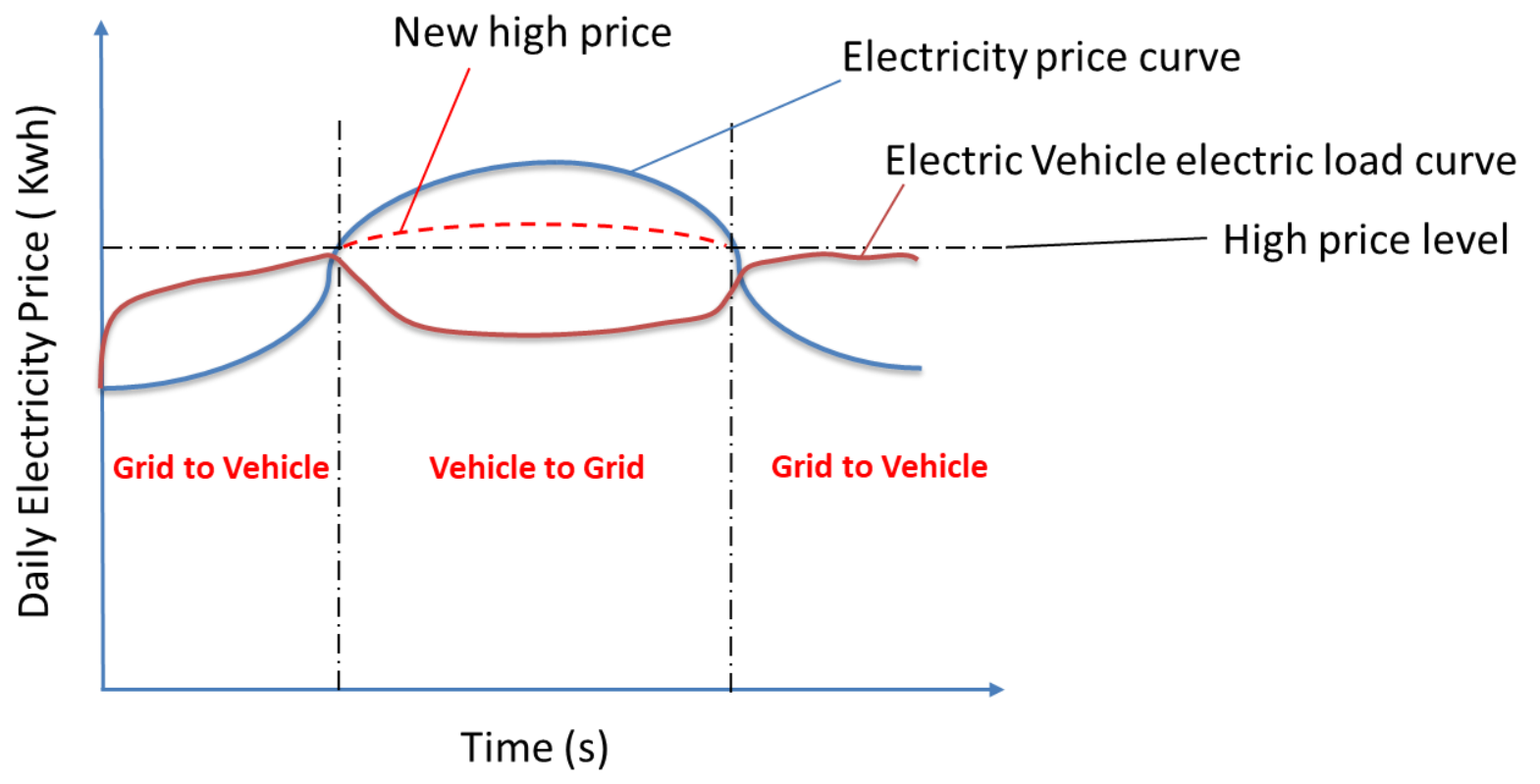

Figure 1.3 Daily Electricity price with and without the usage of EV in V2G mode 


\subsection{Modelling battery chargers}

The battery chargers of the EVs come in different structures. The well-distributed approach in the market is the unidirectional one $[19,20]$. Unidirectional battery chargers can only charge the vehicle. The problem with this approach is that a big amount of EVs requesting power to the can cause a huge drain in the grid, hence, a bidirectional approach has been introduced [21,22]. Bidirectional battery chargers cannot only charge from the grid but also deliver back the energy available in the battery to satisfy the grid requirements at certain periods. This procedure increases the charging times, but it also profits by lower fares.

On the other hand, having all these kinds of chargers in the system without any control in a fleet scale has its drawbacks. The uncontrolled usage of an EV fleet, can lead to several problems such as huge power drains from the grid on the G2V operation and high spikes created from a massive return of energy to the grid on the V2G operation. Therefore, a system-level approach has to be considered in order to avoid any unwanted situation in the bargain with electric power. A Transmission System Operator (TSO), in case of a fleet of EVs entering the grid, will face a problem with the variety of charger structures. As a result, a smart solution that will unify all kind of battery chargers must be applied.

A useful method for modelling a fleet of chargers is the black-box approach. The main feature of the blackbox approach is that it does not need any internal information of the converters to form a model. The black-box model provides a dynamic model by means of system identification methods through the responses to tests applied on the external ports of the charger. The tests can be performed in time or frequency domain.

The interactions between the grid and the battery charger can be well structured with small-signal analysis. This method is simple and easy to implement and it is based on the linearization around a specific operating point through equations. Normally, the interaction between converters (such as battery chargers) and the grid are well defined by a small-signal approximation to keep the power in system level constant with accurate outcome.

On the other hand, small-signal approximation in very variable operating conditions is quite far from the actual behaviour, and a good representation of battery chargers requires nonlinear models. This work is directly focused to develop linear and nonlinear black-box models to obtain an accurate representation of the charger behaviour, either unidirectional or bidirectional in any operating conditions.

\subsection{Contributions}

In summary the main contributions of this thesis are:

1. Modelling of AC/DC battery chargers by identifying the transfer functions in any operating condition without knowing the specific details inside those chargers. This approach is called black- 
box modelling and the strategy is based on the information provided by the input and output variables of the charger. The research illustrates the flexibility of the proposed models to work with single-phase and three-phase AC/DC chargers which are linked to on-board and off-board charging stations.

2. The models proposed in this thesis are applicable in either small-signal and large-signal operation of the battery chargers. Small-signal models are linear, and the large-signal models are based on the Polytopic architecture showing a nonlinear behavior.

3. The proposed models are extensively tested in wide variety of operating conditions showing a good accuracy.

\subsection{Thesis outline}

Chapter 1 has been devoted to introducing the topic and the main objectives and contributions of this thesis. Chapter 2 is showing a survey with different structures of EV battery chargers existing in the market. These chargers can be classified as:

- onboard and offboard chargers

- $\quad$ single and three-phase

- unidirectional and bidirectional

This chapter also explains the different identification techniques used to obtain the linear and nonlinear (polytopic) black-box models.

Chapter 3 is dealing with a design of an AC-DC battery charger in a power simulation program with 3 parts (boost rectifier, dual active bridge and a battery charger) in the first part and then a more complex of a secondary battery added as a second battery charger owner. In the end of this chapter both battery chargers are identified using linear black-box method showing the accuracy between the virtual prototypes of battery chargers with black-box models.

Chapter 4 is dealing with the experimental part of the thesis showing 2 setups both with a commercial battery charger but the first by substituting them with a unidirectional power source and a DC electronic load in a resistive mode and the second by having E-bike batteries. In the last part, in the same manner as in chapter 3 , chapter 4 is using black-box model to identify the experimental setup illustrating once more the accuracy of black-box model but here with actual battery charger setups and the black-box model. The model methods are including linear and nonlinear methods.

Chapter 5 is illustrating the conclusions along with the future work of this thesis document. 



\section{CHAPTER}

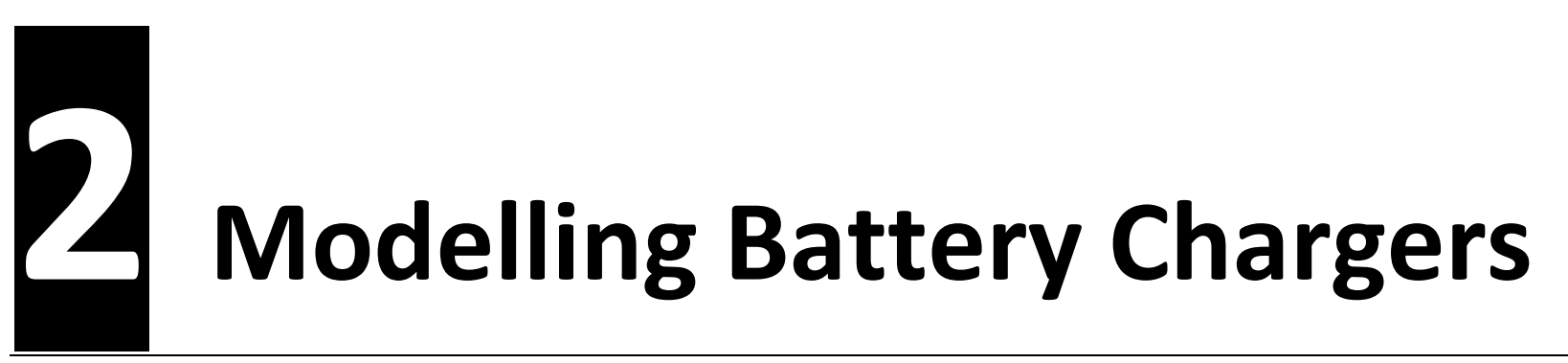

\subsection{Introduction in battery chargers}

As the era of the conventional resources has already started to fade many applications have followed the 'green' route. On this innovation path a huge chapter of conventional application as vehicles could not be absent. In the recent years, different kind of electric vehicles (EV) introduced themselves. The three main EV types in the market are the fuel cells (FCEV) the hybrids (HEV) and the battery EV (BEV).

Hybrids are divided in 2 subsections, the purely hybrids and the plug-in hybrids. Both of them have gasoline engine and electric motor with batteries. Their difference is based on the charging of the batteries which in the case of purely hybrids the batteries can be charged by the combustion engine and the regenerative braking and in the hybrid plug-in, the batteries can also be charged plugging into outlet [23].

Battery electric vehicles in difference with HEV's have only an electric motor with batteries which eliminates emissions from the combustion engine. Also, the BEV can be connected to an outlet or charging stations and gets their batteries charged.

Fuel cell electric vehicles are using the same electric motor structure as battery EV, but the storage of energy is quite different. Instead of using an outlet to charge, a hydrogen tank is added and with the mixture of oxygen from air produces electricity in the fuel cell.

In this subsection different kind of electric vehicle battery chargers will be shown and explained. Due to the high expansion of this technology a large variety entered in the market from the simplest scheme to the more complicated, from low power-single phase- to higher power-3-phase- and from unidirectionalto bidirectional power flow. 
The range of the battery chargers as it was mentioned before varies from a simple scheme of a diode bridge with a capacitor and an inductor [24,25] to a complex 2-stage scheme [26] with an AC-DC converter composed of the cascade connection of a boost power factor corrector converter and a DC-DC converter.

The most known approach of an EV battery charger scheme consists of 2 stages [27,28,29], an AC/DC converter with a power factor corrector (PFC) and a DC/DC converter. The AC/DC converter is used mainly to rectify the $A C$ power to $D C$ and maintains power factor as close to unity as possible. The DC/DC converter is responsible for adapting the voltage and current levels at the requirements of the battery providing galvanic isolation.

Another approach is the single-stage scheme $[30,31,32,33,34]$. In contrast with the 2 -stage, the singlestage has some advantages over the first. The main limitations of the 2-stage are the less efficient power conversion due to the double power conversion and the cost because of the higher component count (e.g. semiconductors). Also, the more complex control structure and a bulky dc-link capacitor are important factors against the 2-stage converter, but on the other hand the 2-stage chargers are more flexible to control the power factor and the battery current independently.

As it has been noticed from the previous references, the majority of the EV chargers lie on the conductive charging. Conductive charging is known to use a direct contact between the charge socket and the vehicle connector through a cable. Recently a significant number of inductive EV charging applications appeared $[35,36,37,26]$. In inductive charging, a transmitting coil transfers energy in the form of AC electromagnetic field and the receiving coil alternate it to electricity to charge the batteries. This approach has some disadvantages in comparison with the conductive charging like the relatively lower efficiency, manufacturing complexity and the cost. On the other hand, it gives more vehicle charging comfort without the use of the cable and also safety through galvanic isolation (Figure 2.1).

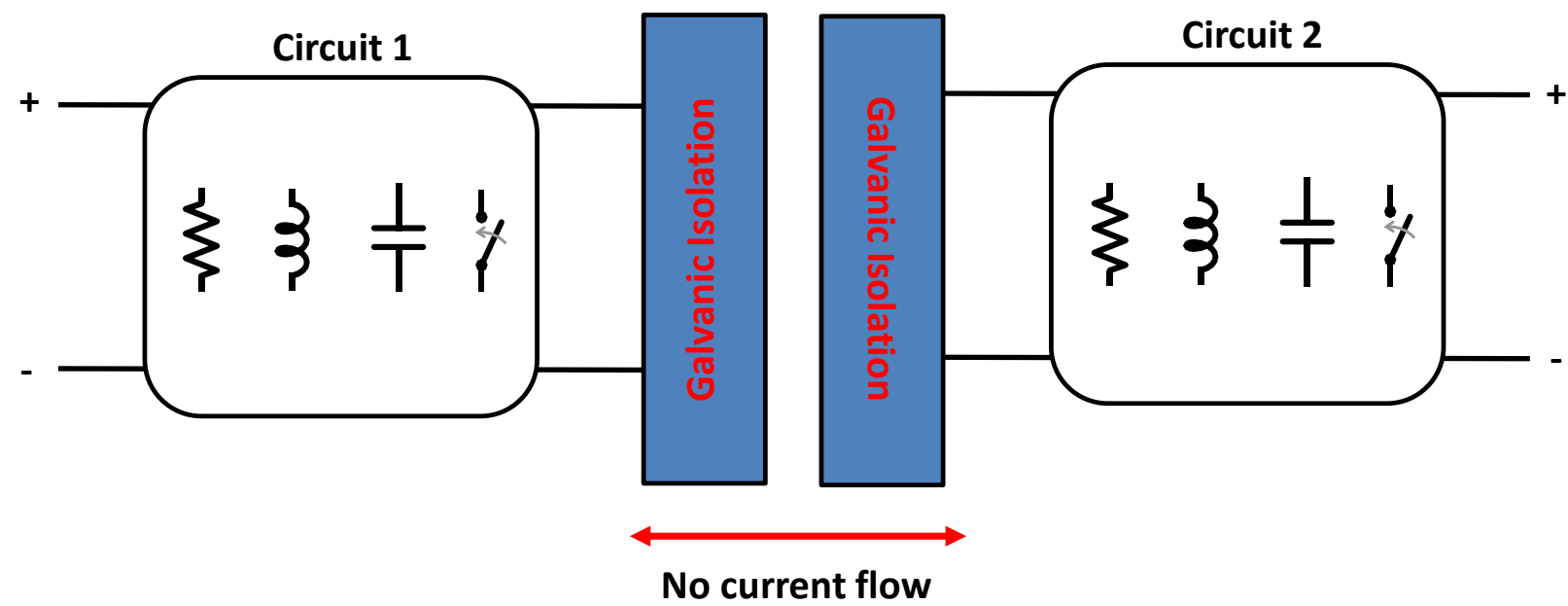

Figure 2.1 Galvanic isolation between two circuit parts 


\subsubsection{On and off board chargers}

EV battery chargers can be also classified depending on where they are placed. On-board chargers are placed inside the vehicle, whereas off-board chargers are outside [38-42]. As it was mentioned in the previous section a typical battery charger consists of an AC/DC converter and a DC/DC converter (Figure 2.2).

In on-board chargers the weight has to be taken into consideration, due to the limited space and also to the extra weight which will burden the vehicle. Consequently, they are designed to work in a low charging rate and, typically, they take several hours to charge. Commonly, they use ac charging stations of level 1 (residential) and level 2 (commercial).

Despite the disadvantages, on-board chargers have important advantages to offer. In a matter of EV battery heating there is much less concern in comparison with the off-board chargers due to lower power levels. Also, the battery management system (BMS) is easier than the case of off-board chargers because the connection and the system is fixed.

Off-board chargers have also remarkable advantages over the on-board ones. Firstly, the higher charging power rate, using dc charging stations, which can complete the charging process in a matter of minutes instead of the hours needed in on-board solutions. Secondly, due to the absence of the charger, the

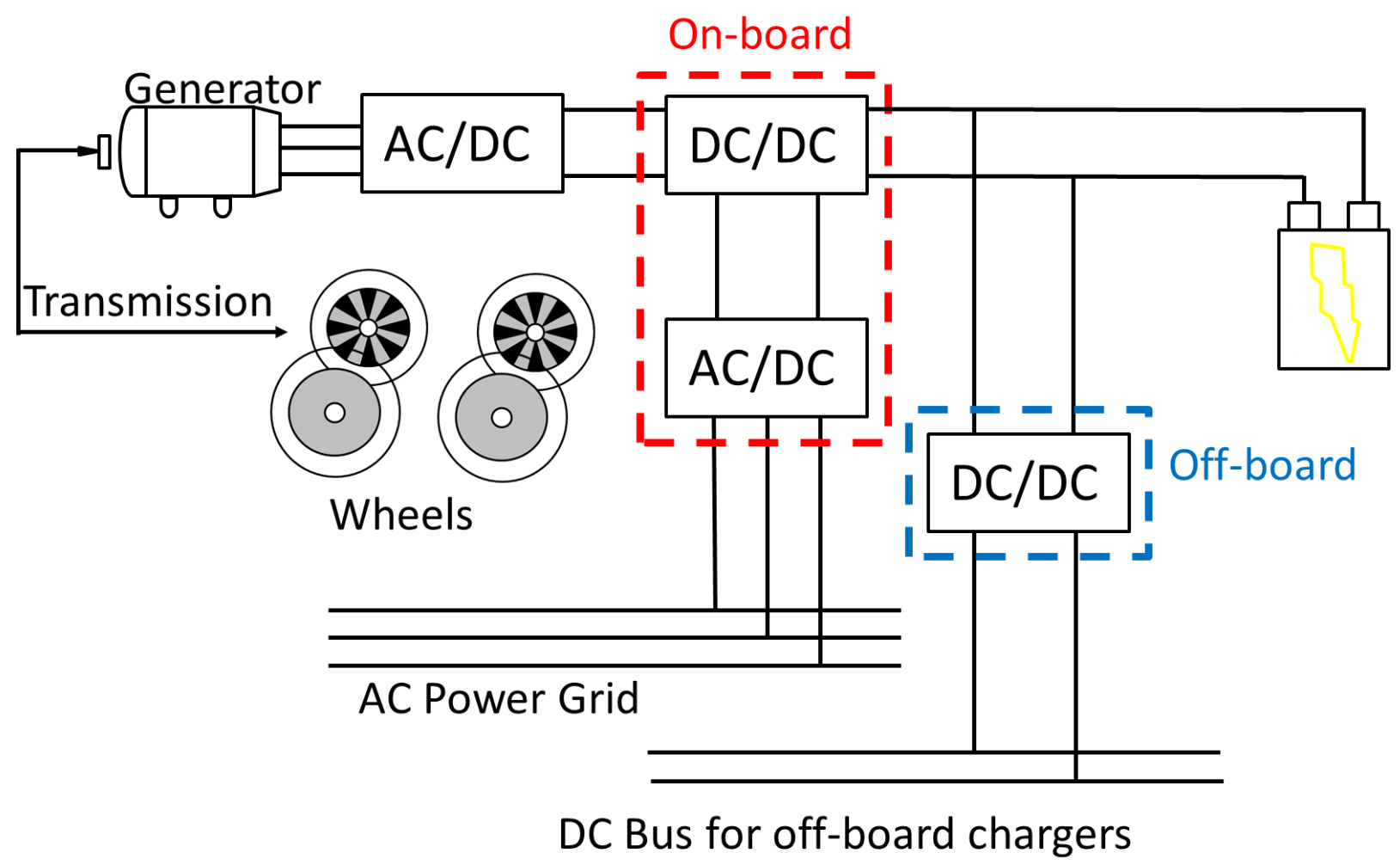

Figure 2.2 Charging of an EV system with both on and off board chargers. 
weight in the car drops significantly and it can increase the vehicle's overall efficiency. Additionally, in this case it is easier to communicate the battery with the grid energy management system.

\subsubsection{Single and three-phase battery chargers}

As it has been mentioned previously, AC charging stations are used for slower charging rating on on-board EV chargers. The AC charging supply is divided in single-phase and three-phase charging stations.

The main difference of single and three-phase supply system is the amount of power that they are able to deliver. Higher power levels in three-phase implies lower charging times and the corresponding extra cost in terms of volume and complexity [43].

On the other hand, residentially, single-phase supply is preferred because there is no need for such threephase distribution that implies an extra cost for the consumer. Changing the electricity infrastructure just for adding a power supply for EVs might not be worth it financially.

\subsubsection{Unidirectional and Bidirectional structure}

The direction of the power flow is also a sorting factor among the battery chargers. In unidirectional battery chargers the power can only flow in one direction, in particular, from the grid to the vehicle (G2V) battery. In contrary, the chargers which can also transfer power to the opposite direction - from vehicle battery to the grid (V2G)- are called bidirectional [26,44-47].

The majority of the EV battery chargers existing on the market are unidirectional. These chargers typically consist of a diode bridge rectifier followed by DC/DC converter. Also, their simple control makes easier the energy management from an amount of EVs.

A typical scheme of a bidirectional battery charger it composes of a bidirectional AC/DC rectifier followed by a bidirectional DC/DC converter. Both unidirectional and bidirectional approaches can use isolated configurations or not. The more complex power structure and control circuitry of bidirectional topologies is a disadvantage in comparison with unidirectional ones.

The bidirectional approach is a relatively new technology. The idea behind this approach is to provide to the owner of the vehicles the flexibility of interacting with the grid and bargain to profit from an energy bargain. Owning a bidirectional battery charger gives the opportunity of having an extra power source available connected to the grid. 


\section{Bidirectional EV's}

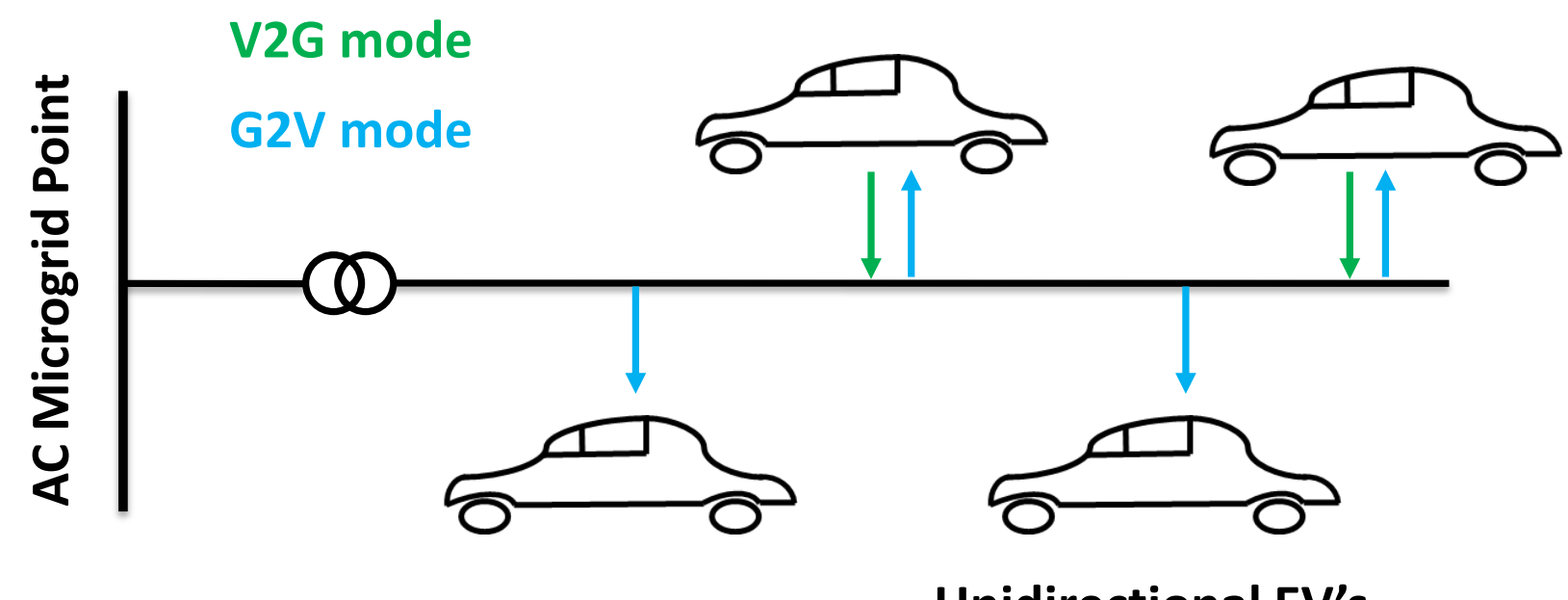

Figure 2.3 Unidirectional and bidirectional EV's interacting in a microgrid

This gives the leverage to distribute the power more efficiently with less power loss. As an example, an amount of energy can be sent back to house -Vehicle to House-for domestic purposes. In a different approach, the Grid Technical System Operator (TSO) can take, occasionally, active, or reactive energy from the vehicles to reduce the peak demand or for stabilization purposes (Figure 2.3). There is also the opportunity to sell and buy energy from the grid when the prices are more in favour of the owner.

\subsection{Modelling Requirements}

In the introduction, it was described that the main motivation of this work is the development of models for battery chargers able to represent their behaviour at system level. Having these models, it would be possible to simulate the grid behaviour, including dynamic analysis, either as loads or generators depending on the G2V or V2G operation. As it was clearly mentioned in the first part of this chapter, electric vehicles (EVs) are being introduced at a fast pace. The variety of EVs existing in the market is as numerous as their battery chargers. Different kind of EV chargers has been described previously putting thoughts on how this amount of EV can be controlled. Controlling this amount of EVs entering the grid is a must for the utilities and the availability of models will contribute to this objective.

Most of the modelling work in the literature has been oriented to DC-DC power converters, although there is a significant amount of work devotes to either DC-AC or AC-DC power converters. On the other hand, most of the proposed models in the literature are linear, assuming generally the small-signal approximation. Linearity is an excellent characteristic that simplifies the modelling problem. 
Unfortunately, the battery chargers, and particularly bidirectional ones, show a huge variation of the operation conditions, even behaving as a load or a generator, and it is possible to switch dynamically between these two different operating modes. For all these reasons, it is required to address nonlinear model structures in order to represent the battery charger behaviour at system level.

On the other hand, most of the work already developed for DC-DC converters can be applied to threephase AC-DC converters using the Park's transformation of three-phase signals to DC signals in the synchronous $d$ - $q$ axis. For single-phase applications, it is also possible to apply a single-phase $A C$ transformation to the $d-q$ axis. Assuming, then, the AC side signals can be transformed to the synchronous reference frame $d-q$ axis, all the powerful modelling structure applied to DC-DC converters could be extended to model AC-DC converters.

In the following sections, some of the most popular linear modelling strategies for DC-DC converters are described.

\subsubsection{Linear Models}

Among different options to model DC-DC converters, a well-established method over the years for representing the dynamic behaviour of switching converters is the state-space averaging. This technique which is used by industry and academia is proven to be highly capable of representing the converters dynamic behaviour in continuous and discontinuous conduction modes. The small signal average model procedure is created through the stages of averaging, perturbation, and linearization. This approach requires a detailed knowledge of the converter to be modelled.

The stages of creating a state-space averaging model of a DC-DC converter in continuous conduction mode will be described below [48-49]. Firstly, a state-space description is separated in linear circuits corresponding to each switching period. Those linear circuits are alternatively operating in the ON and OFF state of the switch transistors creating a number of consecutive linear intervals. The set of equations is averaged using the duty cycle in order to create the state-state averaged behavioural model. The next step is to linearize the state space equations around an operation point, which can be performed by adding a small perturbation, expanding the result and eliminating the nonlinear values -which are produced by the product of small perturbations of two different variables.

A completely different approach to obtain a converter's averaged behavioural model is system identification. The system is settled to an operation point and analysing the response of the converter to different tests set to the input variables, a small-signal input-output model is obtained. The determination procedure of a model starts adding a small perturbation to each of the input signals. The response that comes from the output accompanied by the input perturbation are used for the construction of a transfer function linked with that operating point. The input/output values which are collected have before subtracted their nominal values. The same operating point has to be applied on every input but separately 
in order to get accurate transfer functions. Making this step implies the assumption of the linearity of the system, as the superposition theorem is applied. The identification process is applicable in both time and frequency domain.

In linear models the identification methods can be separated in parameter and non-parametric methods [50]. Parameter estimation methods are the methods which identify several parameters in a model structure. Examples of this kind of structures are grey and black-box models described below

The modelling approaches are separated in three levels of knowledge: the white, the grey and the blackbox [51-56]. In the upper part of the Figure 2.4 it can be clearly seen the power flowing through the battery to the charger (DC/DC and AC/DC), then to the generator and lastly to the wheels. This modelling approach applies only when the converter structure and control are completely known. Then, it is possible to derive the differential equations that describe its dynamical behaviour and to use the state-space averaging technique for modelling the system. This is the typical approach to obtain the white-box model.

The middle part of Figure 2.4 shows partly the EV operation. The same applies on the grey modelling approach. In this model the physical insight is partly known but there are several parameters that have to be determined from testing data. Lastly the black-box model is the one used when no insight data is given, and the parameters are determined through observed data.

In practice, most of cases when you try to model the battery charger in the vehicle, the available information is very limited and the situation is rather close to the black-box models described in the previous paragraph. On the other hand, the number of different vehicles and their corresponding onboard chargers is so high, that it is impossible to have insight information of them. The same conclusion can be applied to the off-board chargers, coming for a high number of different manufactures offering a very limited information about their internal structure (power stage, control loops, etc.).

\subsubsection{Small-Signal Black-box Behavioural Model}

Although, the battery charger behaviour is nonlinear and consequently a good accurate representation of the converter requires a nonlinear model, the first step is to define a small-signal black-box modelling structure, which has been proposed in this thesis. As it was mentioned before, assuming the small-signal conditions are set, any nonlinear system can be approximated with a linear system. This model takes advantage of previous work oriented to obtain behavioural black-box models for DC-DC [57] and DC-AC [58] converters. This extension to the AC-DC converters, in general, and to battery chargers, in particular, is one of the main contributions of this thesis [59]. 

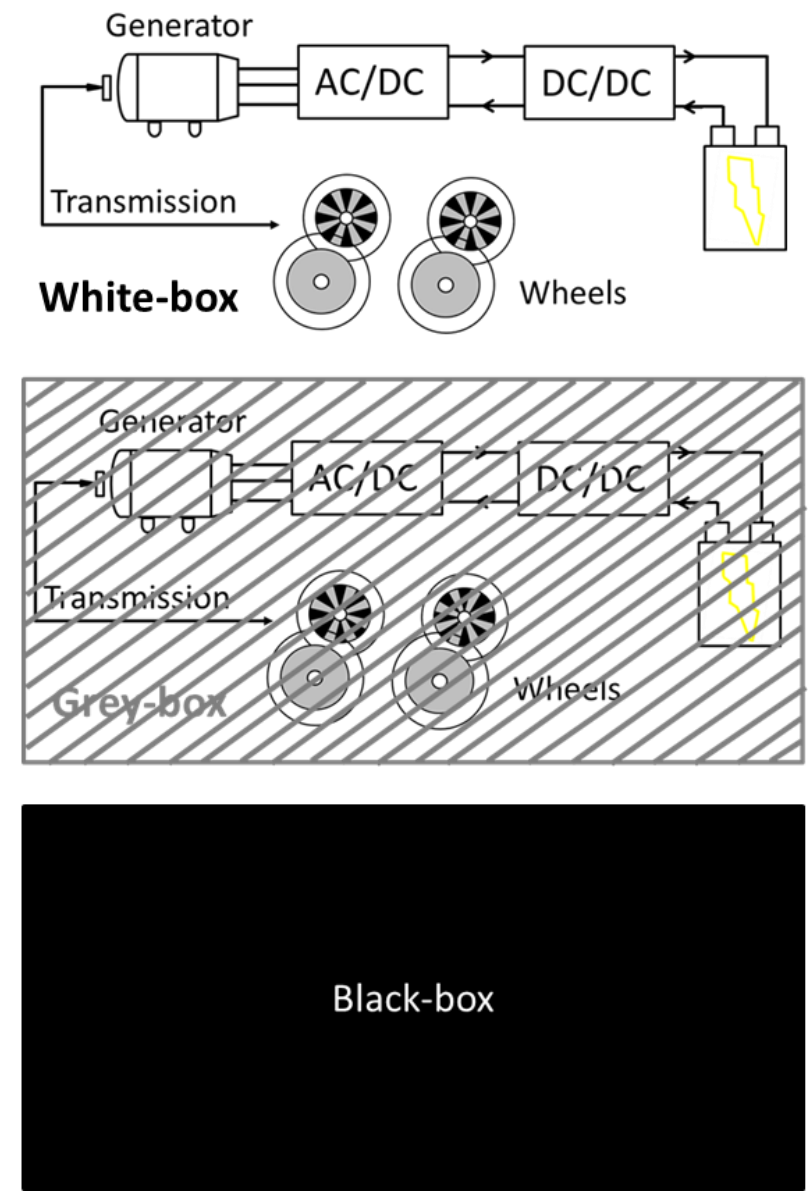

Figure 2.4 White, grey, and black-box model concepts.

Black-box model structures are widely known from the DC/DC converters [58, 60-64]. The extension from DC/DC structure to AC/DC structure requires a Park transformation. A typical AC/DC battery charger system is connected to a three-phase $A C$ input. Their input variables are recognised as the input ( $\left.V_{a b c}\right)$ voltages along with the control reference $\left(I_{\text {ref }}\right)$, while the output variables are the input $\left(I_{\text {abc }}\right)$ and output $\left(I_{\text {bat }}\right)$ currents.

In Figure 2.5 the battery charger transformation is shown from the AC to $D-Q$ framework. The upper part depicts a three-phase system, whereas the lower part represents a single-phase system [65]. The threephase case has the $A C$ variables transformed to $D C$ in the $D-Q$ framework through Park transformation. The DC model new variables of voltage and current which are the $d$ and $q$ components $V_{d}, V_{q}$ and $I_{d}, I_{q}$ from their respective $A C$ values $V_{a b c}$ and $l_{a b c}$. The values of the DC model are completed with the input reference current and the battery current output. 
The single-phase AC/DC converters model transformation follows partly the procedure of Park transformation. The park transformation consists of Clarke transformation which translates an AC threephase frame $(a b c)$ to an $A C$ two-phase frame $(\alpha \beta)$ following by a rotation to $D-Q$ framework (dq). Since in the single-phase case it is not possible to apply the same route, the procedure splits. By considering that $V_{a}$ is in phase with the $V_{\alpha}$ an artificial $90^{\circ}$ phase shift is applied to generate the beta component, $V_{\beta}$. After the creation of the $\alpha \beta$ plane a rotation is applied creating the $D-Q$ framework. The variables in the singlephase structure in the Figure 2.5 (b) are the same as in (a) with the difference of the AC part having only $V_{a}$ for voltage input and $l_{a}$ for current output.

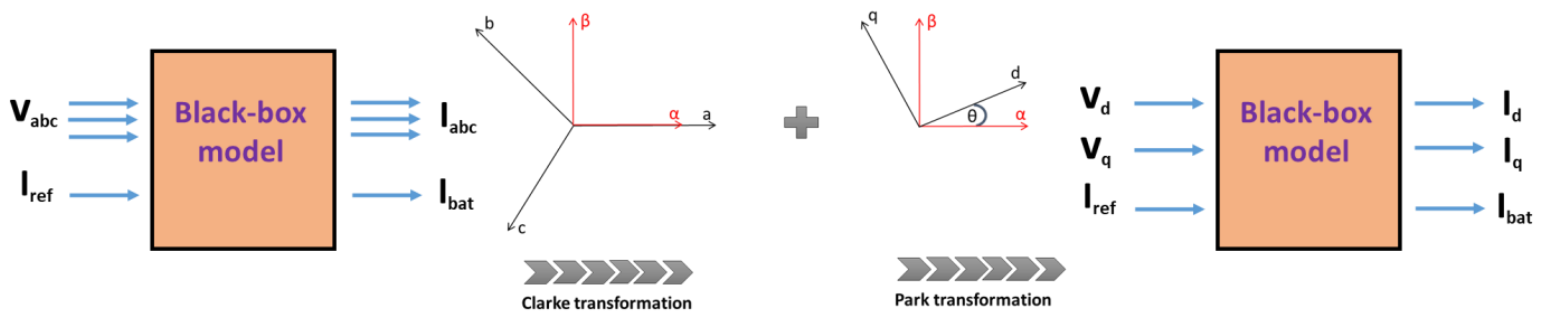

(a)
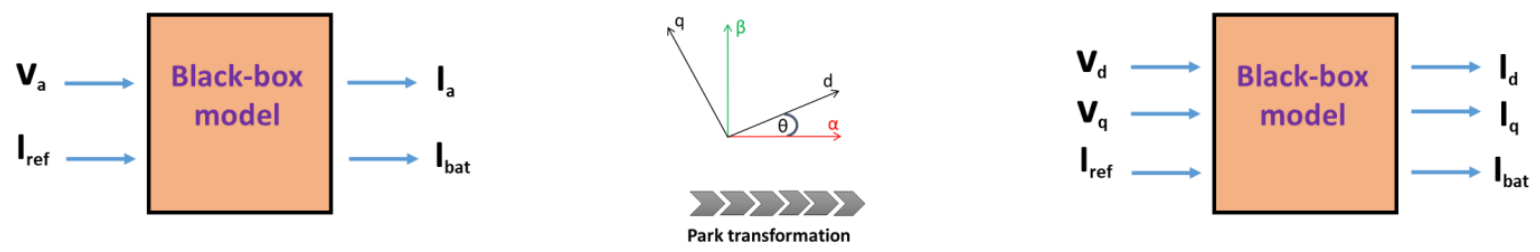

(b)

Figure 2.5 Battery charger transformation to D-Q framework: a) 3ph AC/DC battery charger transformation to $D-Q$ framework, b) 1 ph AC/DC battery charger transformation to $D-Q$ framework
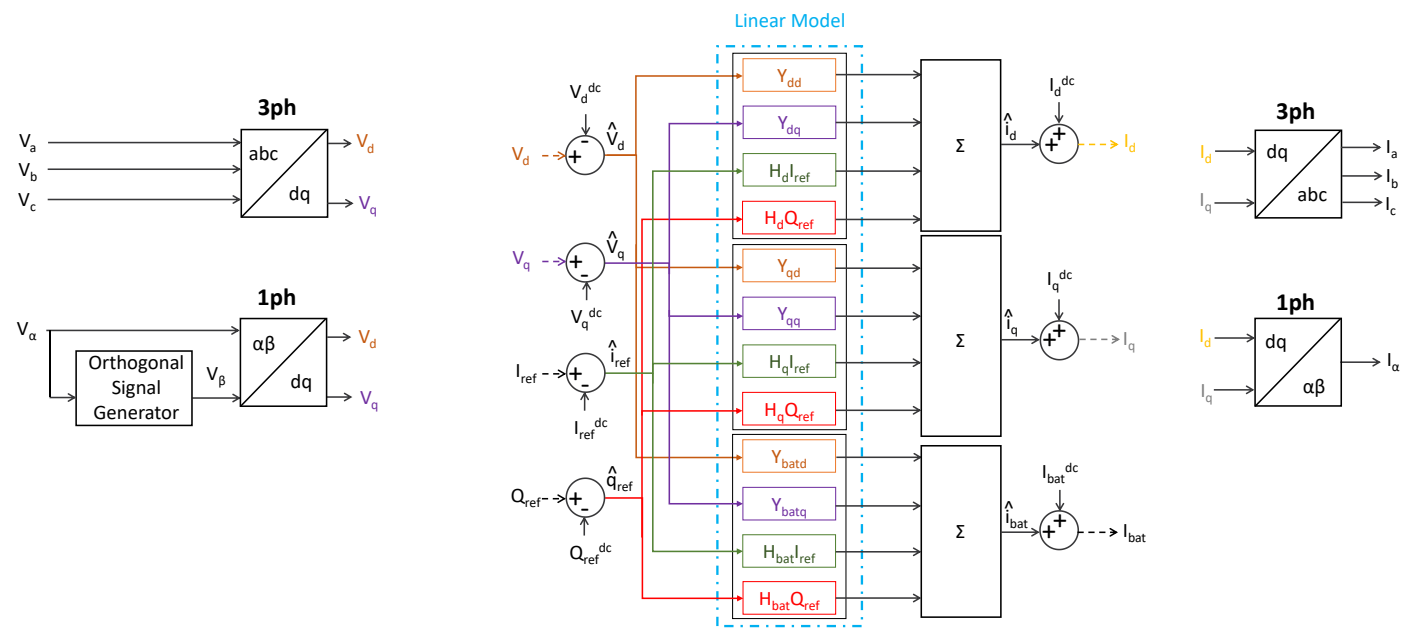

Figure 2.6 Block diagram of the black-box model 
In case of small-signal conditions meeting around an operating point, then the system could be considered linear, and the small-signal black-box model can be obtained. Adding a small-signal step perturbation in one of the input variables and observing the perturbation in the output variables, a collection of transfer functions is easily obtained through identification algorithms. Following the same procedure with all the input variables, it can be obtained a number of transfer functions equal to the number of output variables multiplied by the number of input variables. As linearity is assumed, the superposition theorem can be applied, and the small-signal model structure represented in Figure 2.6 can be obtained. It must be taken into account that the variables are perturbations of the input and output variables around the operating point, and they are represented by $\hat{x}$ letters.

The following matrix equation (E 2.1) represents the small-signal model of the block diagram described in Figure 2.6, where, $Y_{d d}(s), Y_{d q}(s), Y_{q d}(s), Y_{q q}(s), Y_{\text {batd }}(s), Y_{b a t q}(s), H_{d I}(s), H_{q I}(s), H_{b a t I}(s), H_{d Q}(s)$, $H_{q Q}(s)$, and $H_{b a t Q}(s)$ are the transfer functions that describe the dynamic response of the system, where the first subindex refers to the output and the second subindex refers to the input variable involved. For instance, $Y_{d q}(s)$ is the transfer function that describes the dynamic response of the d-component of the input current when the q-component of the input voltage is perturbed and the rest of the input variables are kept constant. The rest of the transfer functions can be defined analogously. The small-signal model defined in (1) can be represented by the equivalent circuit depicted in Figure 2.7.

$$
\left(\begin{array}{c}
\hat{\imath}_{d}(s) \\
\hat{\imath}_{q}(s) \\
\hat{\imath}_{\text {bat }}(s)
\end{array}\right)=\left(\begin{array}{cccc}
Y_{d d}(s) & Y_{d q}(s) & H_{\text {dref }}(s) & H_{d q}(s) \\
Y_{q d}(s) & Y_{q q}(s) & H_{\text {qref }}(s) & H_{q q}(s) \\
Y_{\text {batd }}(s) & Y_{\text {batq }}(s) & H_{\text {batref }}(s) & H_{\text {batq }}(s)
\end{array}\right)\left(\begin{array}{c}
\hat{v}_{d}(s) \\
\hat{v}_{q}(s) \\
\hat{\imath}_{\text {ref }}(s) \\
\hat{q}_{\text {ref }}(s)
\end{array}\right)
$$

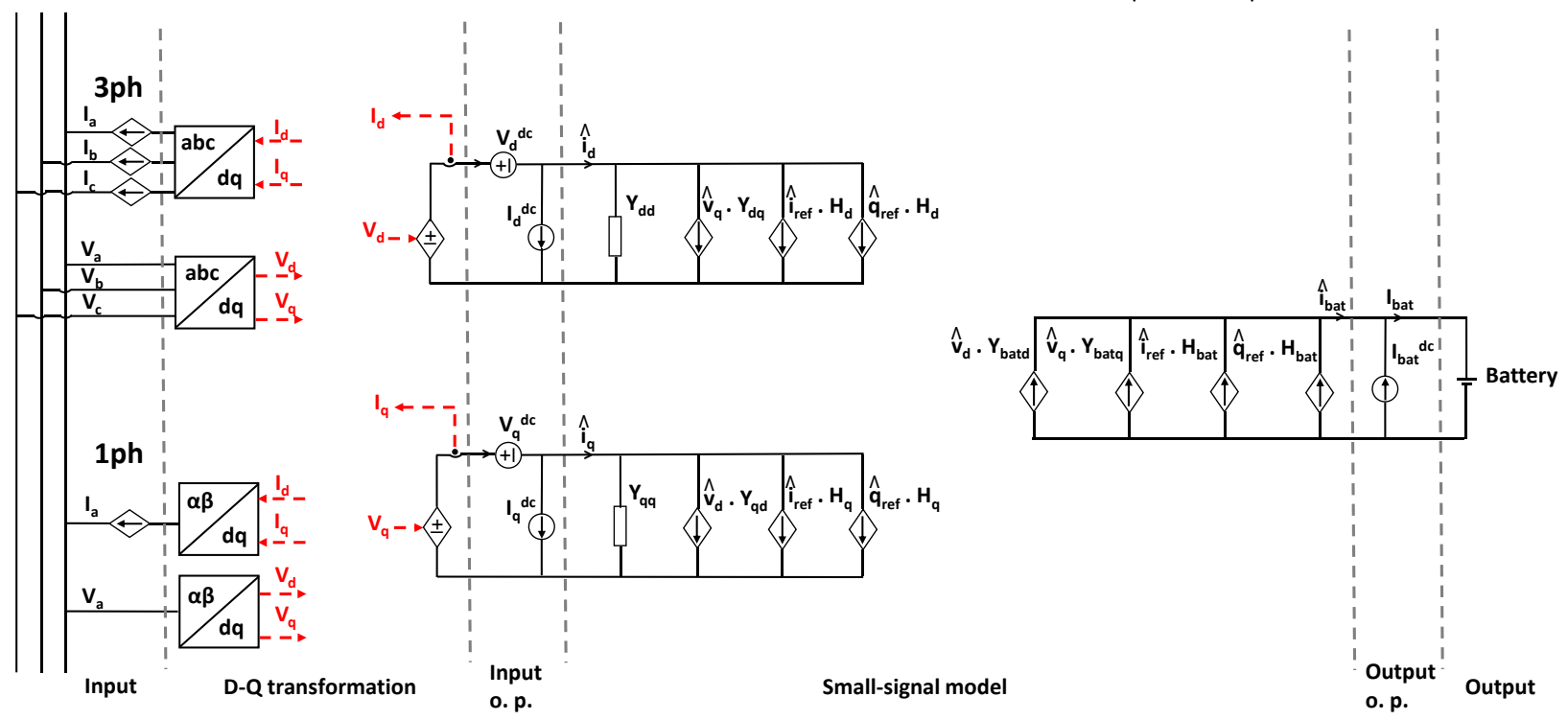

Figure 2.7 Equivalent circuit of the black-box model 
The limitations of this model come from the definition of small-signal average models and the use of the $D-Q$ synchronous reference frame. Therefore, this model is useful for systems with a linear response or nonlinear systems around a specific operating point, which is considered as a dc bias in the model. On the other hand, it does not consider the switching ripple, but it provides information about the average dynamic performance of the system. The synchronous reference frame restrains the model to balanced conditions of the grid and it only provides useful information about its fundamental frequency.

\section{Modelling procedure}

The procedure to obtain the model is as follows. First, an operating point is selected, defined by the values of the inputs and outputs variables in steady state. Then, associated to this operating point, a small-signal model is generated. This small-signal model is characterized by twelve transfer functions as in the block diagram of Figure 2.6. To obtain these twelve functions, small-signal steps around the operating point values are applied to every input, one by one, observing the perturbation in the output variables, while the rest of the input variables are kept constant. With this information by means of identification algorithms in MATLAB, the transfer functions represented in the block diagram of Figure 2.6 are generated. The order of the transfer functions identified should be the lowest value that provides the highest degree of accuracy. The order selection often becomes a trade-off between accuracy and complexity.

The model structure proposed has four inputs: $v_{d}, v_{q}, I_{r e f}$, and $Q_{r e f}$. Therefore, four tests are necessary to obtain the transfer functions that describe the system. The first test should perturb the $d$-component of the input $A C$ voltage, while the q-component is kept constant, as well as the references of the battery current and reactive power. The input AC voltage in a balanced three-phase system can be defined as follows (E 2.2, E 2.3, E 2.4):

$$
\begin{aligned}
& V_{a}=V_{p} \cdot \cos \left(2 \pi f_{\text {grid }}+\theta\right) \\
& V_{b}=V_{p} \cdot \cos \left(2 \pi f_{\text {grid }}-2 \pi 3+\theta\right) \\
& V_{c}=V_{p} \cdot \cos \left(2 \pi f_{\text {grid }}+2 \pi 3+\theta\right)
\end{aligned}
$$

The equations are explained as follows. $V_{a}, V_{b}, V_{c}$ are defined as the phases of the input three-phase $A C$, the amplitude of the phase voltages as $V_{p}, f_{\text {grid }}$ as the grid frequency and the $\theta$ as the angle between the $V_{a}$ phase of the three-phase system and the $V_{d}$ component in the $D-Q$ framework as it shown in figure 2.8 (a). From the above explanation it can be defined the $d$-q components of input voltage as follows:

$$
V_{d}=V_{p} \cdot \cos (\theta)
$$




$$
V_{q}=V_{p} \cdot \sin (\theta)
$$

$V_{p}$ and $\theta$ are defined through the values of $V_{d}$ and $V_{q}$ as it is shown in the (E 2.11) and (E 2.12) in order to perform steps in the $d$-component of the $d$-q framework with q-component remaining constant and the opposite.

$$
\begin{aligned}
& V_{p}=\frac{V_{d}}{\cos \left(\operatorname{atan}\left(\frac{V q}{V_{d}}\right)\right)} \\
& \theta=\operatorname{atan}\left(\frac{V_{q}}{V_{d}}\right)
\end{aligned}
$$

An example of this operation is shown in figure 2.8 (b) and (c). In figure 2.8 (b), the amplitude of phase voltage changes from $V_{p}$ to $V_{p}^{\prime}$ by changing the $V_{d}$ to $V_{d}^{\prime}$ and keeping the $V_{q}$ constant. The operation in figure 2.8 (c) is reversing with having the $V_{q}$ increasing to $V_{q}^{\prime}$ and moving the $V_{p}$ to $V_{p}{ }^{\prime \prime}$ while the $V_{d}$ is constant.

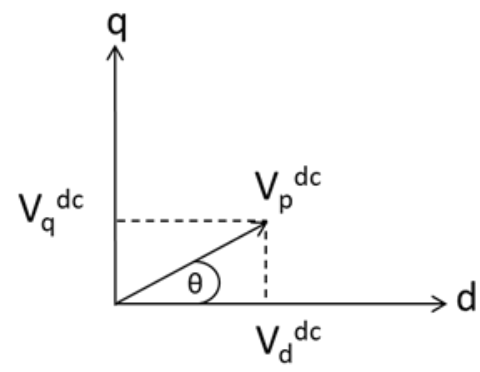

(a)

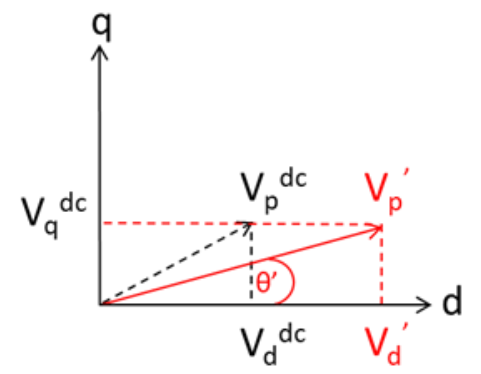

(b)

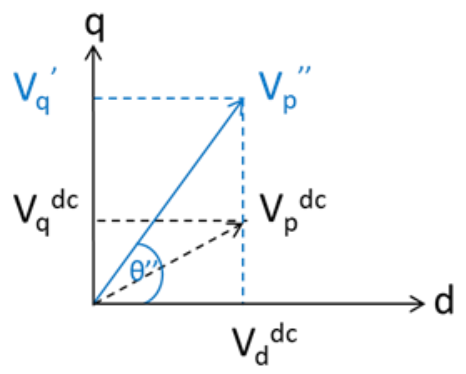

(c)

Figure 2.8 Three phase AC input voltage in the D-Q framework: (a) Operating point, (b) Step in the $d$ component, (c) Step in the q-component.

In many cases, the use of a PLL (Phase-Locked Loop) makes $V_{q}$ equal to zero. In those cases, $V_{d}$ can be stepped by just making a step in $\mathrm{V}_{p}$; and the input $\mathrm{V}_{\mathrm{q}}$ and the transfer functions related to it $\left(Y_{d q}(s)\right.$, $Y_{q q}(s)$, and $\left.Y_{b a t q}(s)\right)$ can be neglected. This simplification further reduces the number of transfer functions of the small-signal model to nine. The third test should perturb the reference of the battery current ( $\left.I_{\text {ref }}\right)$, while the $d$ and q-components of the input AC voltage and the reference of the reactive power are kept constant. Similarly, the fourth test should perturb the reference of the reactive power $\left(Q_{\text {ref }}\right)$, while the $d$ and q-components of the input $A C$ voltage and the reference of the battery current are kept constant. 


\subsubsection{Parametric estimation methods}

In the previous section, the tests needed to identify AC-DC battery chargers were described. In this section, the different methods available to obtain the transfer function that represent the measured response to the perturbations applied to the input variables are detailed. The main difference among them is the way in which they deal with the noise present in the measured signals.

\section{[A] Linear system identification}

Parametric black-box models can be obtained with different type of model structures. Four common examples of those structures are shown in the Figure 2.9 [66]. The first model structure is called AutoRegression $A(q) y(t)$ with extra input $B(q) u(t)$ or $A R X$, whereas $e(t)$ the noise (might correlate it as a switching ripple or measuring noise), $u(t)$ the input and $y(t)$ output signal. In the Figure 2.9 the polynomial $A(q)$ is used as a denominator both for the undisturbed system and the noise model giving the opportunity to be a good choice when both model and system have similar dynamics. ARX equation is shown in E 2.13.

The ARX model structure is quite simple, however, its main disadvantage is the lack of satisfactory freedom in terms of describing the properties of the disturbance term. This flexibility is available through the next model structure, Auto-Regression Moving Average with extra inputs (ARMAX). The moving average is added in the equation with the polynomial $C(q)$ in the noise model and giving more flexibility in the structure. This model has a good use in system description and control design in control and econometrics. ARMAX equation is shown in E 2.14.
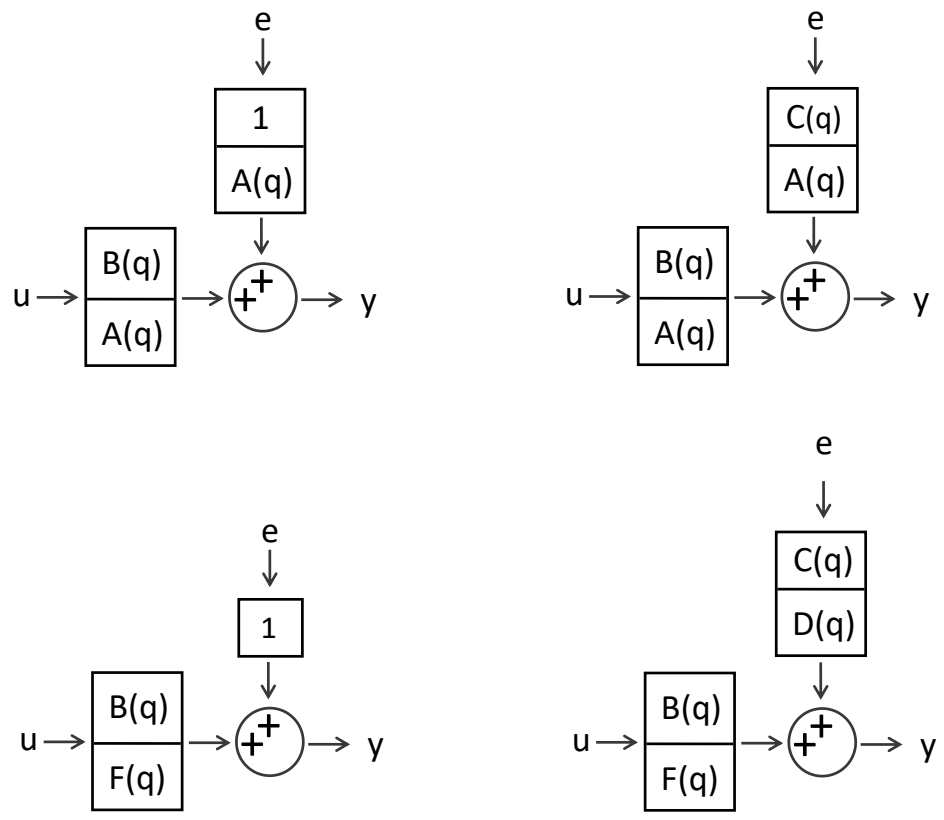

Figure 2.9 Up-left: ARX model structure, Up-right: ARMAX model structure, Down-left: OE model structure, Down right: BJ model structure 
The main difference of the next model compared with the previous ones, is that it does not have a model for the noise. Output Error model (OE) has the least number and the lower order of polynomial parameters that needs to operate with $\mathrm{B}(\mathrm{q})$ and $\mathrm{F}(\mathrm{q})$ as polynomials, which define the transfer function, $y(t)$ and $u(t)$ the output and input signal of the system, whereas $e(t)$ the noise (E 2.15).

Amongst the model structures in Figure 2.9 the most independent in terms of system and noise model is the Box Jenkins (BJ) and because of that the most flexible. The flexibility achieved in this model is because all the polynomials $(B(q), C(q), D(q), F(q))$ do not appear more than once in the equation $E$ 2.16.

$$
\begin{aligned}
& y(t)=\left[\frac{B(q)}{A(q)}\right] * u(t)+\left[\frac{1}{A(q)}\right] * e(t) \\
& y(t)=\left[\frac{B(q)}{A(q)}\right] * u(t)+\left[\frac{C(q)}{A(q)}\right] * e(t) \\
& y(t)=\left[\frac{B(q)}{F(q)}\right] * u(t)+e(t) \\
& y(t)=\left[\frac{B(q)}{F(q)}\right] * u(t)+\left[\frac{C(q)}{D(q)}\right] * e(t)
\end{aligned}
$$

Similarly, to the discrete-time error models mentioned above, there is also the continuous-time model ( $E$ 2.17). The link between the system model is defined by a ratio of polynomials, where denominator polynomial is linked to the number of poles and numerator to the number of zeros.

$$
y(t)=\left[\frac{\text { numerator }(s)}{\text { denominator }(s)}\right] * u(s)+e(s)
$$

After describing a handful of linear black-box models, the selection of the linear models has been limited to two cases. The first linear model is the Output Error model from discrete-time. The idea of choosing this polynomial model structure as the most accurate is due to best-fit response and lowest order of the linear polynomial parameters (E 2.15). The second linear model used in the main core of the thesis is the continuous-time model (E 2.17). As it can be seen the structure of continuous-time polynomial controls only the input part and not the noise. Also, as a continuous model it evaluates more functions of data, which is gives more accuracy than the discrete-time model and that it is shown form the final prediction error (FPE). 


\subsubsection{Nonlinear models}

In the linear model a perturbation around an operating point of the respective input reference can be represented accurately. In the case of larger change in an input step, the dynamic behaviour changes and a mismatch arises between the small-signal black-box model and the actual converter. Therefore, a nonlinear approach has to be followed.

There are several examples of nonlinear modelling approaches and one of the most common is called block-oriented models [67-68]. The idea of block-oriented models is based on the interconnection of linear and nonlinear blocks. A dynamic nonlinear block-oriented model is the nonlinear autoregressive exogenous model (NARX). This method is a combination of a linear function ARX in parallel with a nonlinear function, mapping the regressor output to the output of the model.
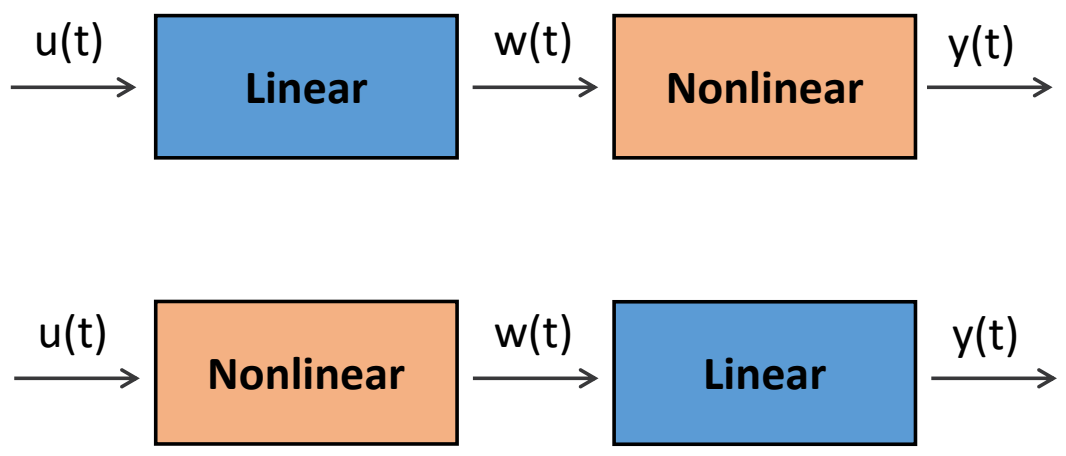

Figure 2.10 Up: Wiener model structure, Down: Hammerstein model structure

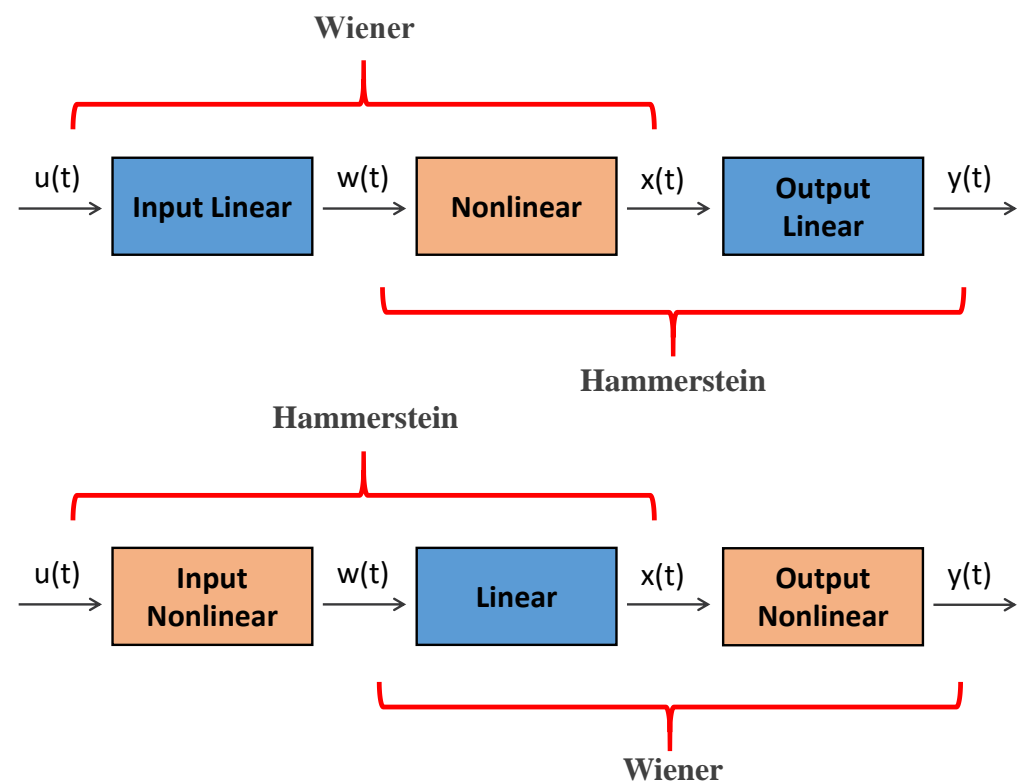

Figure 2.11 Up: Wiener-Hammerstein model structure, Down: Hammerstein-Wiener model structure 
The Hammerstein and Wiener models are two block-oriented models, which have the opposite structure (figure 2.10) [68]. In the Wiener model a linear model is placed to represent the system dynamic followed by a nonlinear block showing the static operating point able to describe linear dynamic operations supplied with nonlinear sensors. On the contrary, in the Hammerstein model, the nonlinear block is placed first followed by the linear model, this structure is able to describe linear dynamic operations guided by nonlinear actuators.

A mixture of the two models with both orders Hammerstein-Wiener and Wiener-Hammerstein are also typical options (Figure 2.11) [68-71]. Hammerstein-Wiener model which describes dynamic systems is composed from static nonlinear blocks placed in the input and output in series with an output-error linear block distorted from static linearities. On the other hand, Wiener-Hammerstein, linear dynamic blocks are placed in the input and the output responsible of the high frequency and transient behaviour, respectively. A non-linear static model is connected in the middle of the two linear blocks representing the behaviour of the converter in steady state, as it is described in the work of Oliver et al. [72]. The nonlinear blocks of Hammerstein-Wiener and Wiener-Hammerstein model structures are defined by nonlinear estimators. Two examples of estimators are the sigmoid and wavelet network [68], both of them are using identification based on neural network.

Typical neural network is composed of several interconnected simple processed units forming a complex network $[73,74]$. Multi-layers of this kind of units are configured so as the data after entering the input layer to meet one or more hidden intermediate layers before finding the output layer. These neural networks are defined as non-linear black-box structures applied due to their potential of non-linear estimation with parameter estimation methods.

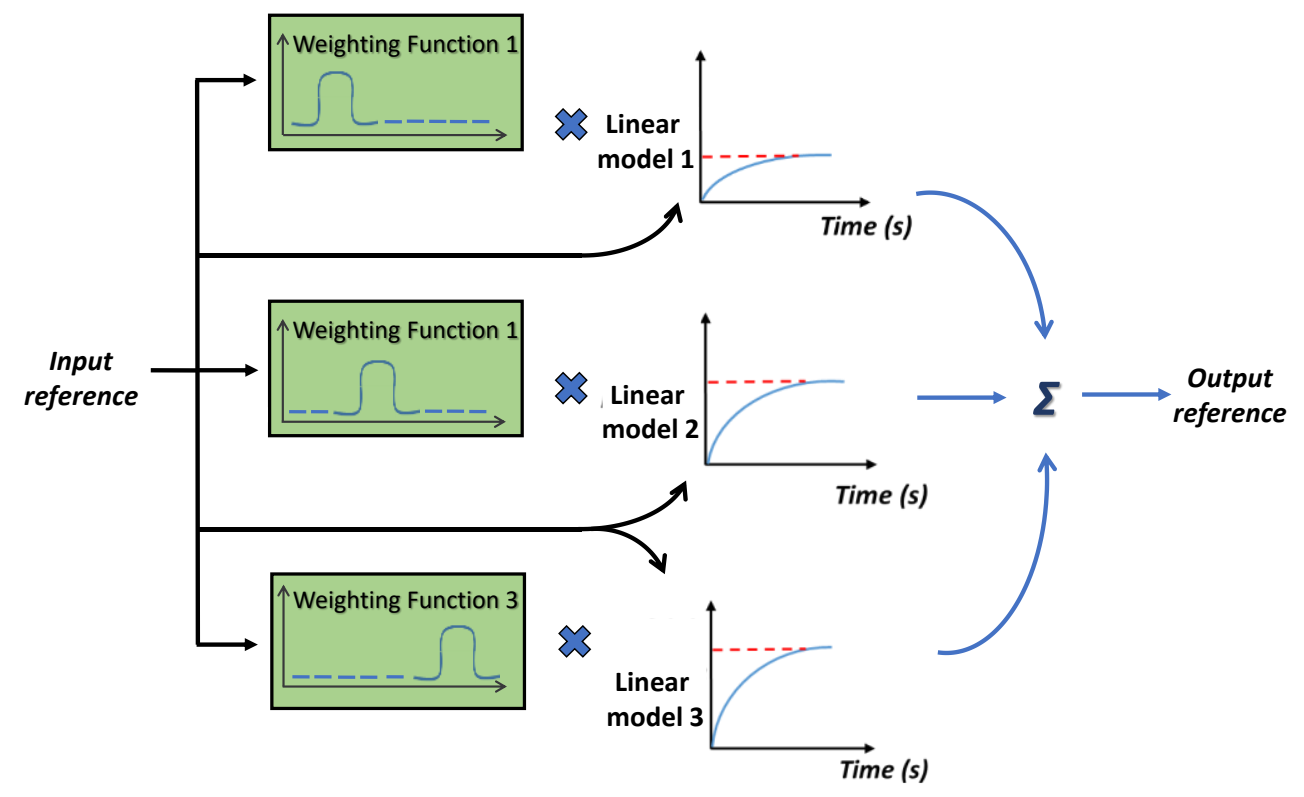

Figure 2.12 Polytopic model structure 
In case of sigmoid network estimator, it can be seen a mixture of radial ground of network function operating a sigmoid as activation function. In the wavelet network case, it can be seen a feed-forward neural net having as an activation function operated by wavelet. The wavelet theory along with neural networks is giving the wavenet estimator, a nonlinear function which is the term outlining the wavelet networks [68].

Furthermore, another modelling approach able to account for dynamic nonlinear behaviours is the polytopic model approach [61,75-76] (Figure 2.12). In comparison with the other techniques, this approach is able to include nonlinearities in the transient dynamic behaviour, not only in the stationary part.

The idea of this model is the combination of different linear time invariant (LTI) models obtained in different operating points covering the range of variation of the input variables. Every linear model is multiplied by an individual weighting function covering the nonlinearities of the converter connecting the previous and the next linear model with the current one. In the end all of those combinations are added together to make a smooth transition among the response of the different linear models related with the different operating points, which gives a more accurate result.

\subsubsection{Large-signal Black-box Behavioural Model}

In this section, the application of the polytopic model approach to the case of battery chargers is described. This structure consists of several linear models obtained in different operating points. In the figure, these linear models are represented by their small-signal equivalent model (Figure 2.7). The output of the large-signal model is a weighted combination of the outputs of the linear models, where the weighting functions can be nonlinear to capture the large-signal behaviour of the converter. A wide range of weighting functions are suitable for this purpose, from the triangular functions used in Takagi-Sugeno models, to more elaborated alternatives as double sigmoid functions [57].

The polytopic model has a high degree of freedom, which allows it to capture the battery charging behaviour. Basically, it depends on the number of variables considered, the number and location of the operating points selected and the type of weighting function.

The number of variables and operating points is a function of the degree of variability of the input variables (Figure 2.13). In our application, the battery chargers, the inputs variables are typically the AC (single-phase or three phase) input voltage, the battery current reference and the active and reactive power references, which implies a high number of operating points. Therefore, depending on the application, it is common the assume that some of these inputs are constant in order to simplify the analysis. For instance, very often the reference of reactive power is set to zero permanently. Furthermore, in case the $\mathrm{AC}$ input voltage is the grid, it can be considered constant. This means that assuming that the $A C$ input voltage and 


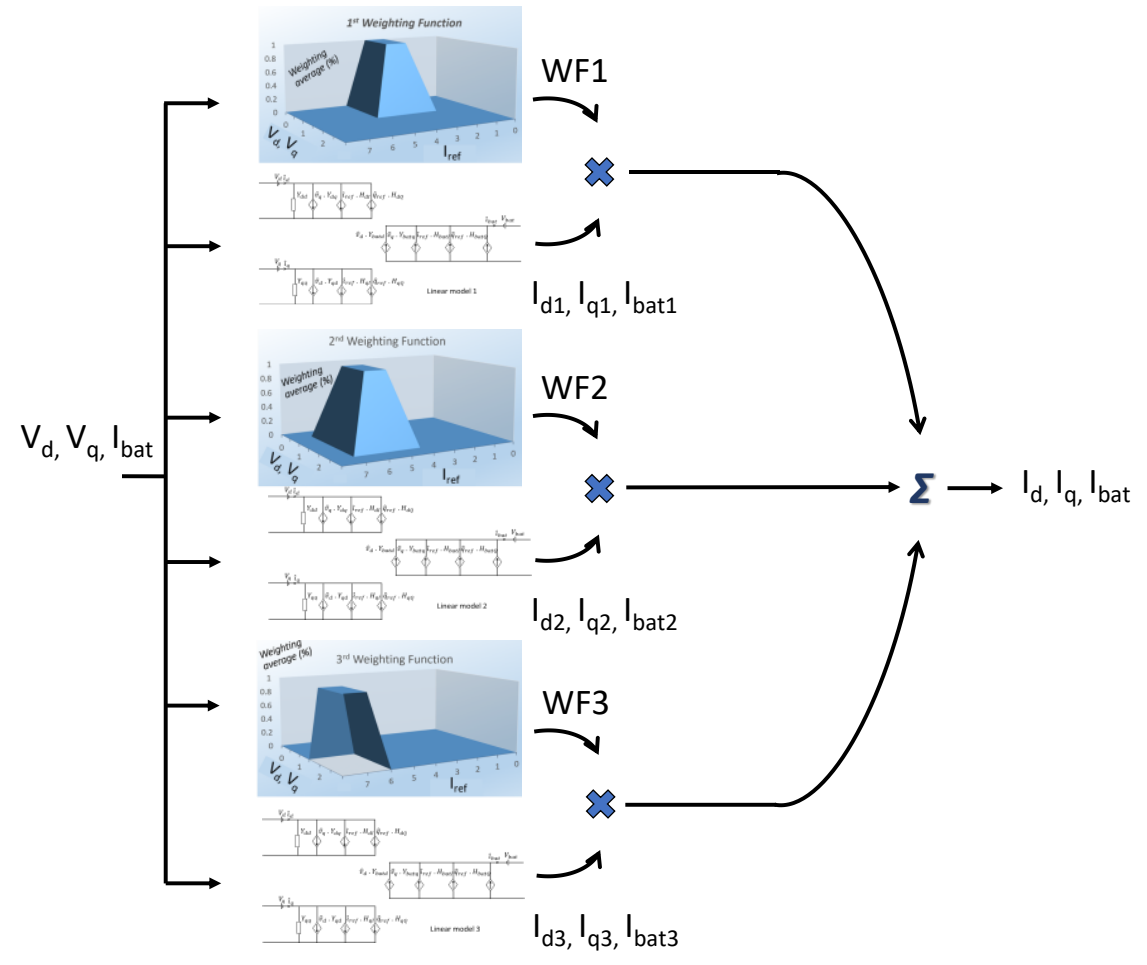

Figure 2.13 Detailed structure of polytopic model structure

the reference of reactive power remains constant, the variability is restricted to the value of the current reference.

Consequently, in the case of battery chargers connected to the grid, several linear black-box models obtained for different values of the set point for the battery current, combined with the nonlinear weighted functions in a polytopic structure is a good approach to capture the large-signal behaviour of the charger. It should be taken into account that, as it is assumed that the input voltage and the reference

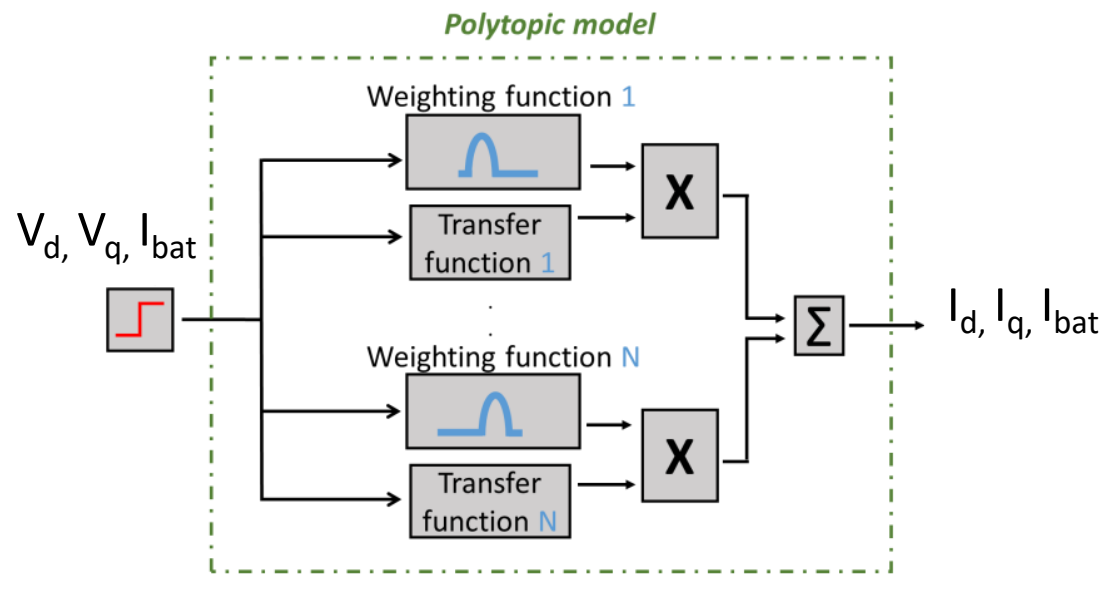

Figure 2.14 Simplified Polytopic model 
for the reactive current are constant, the model is not able to capture the dynamics caused by the changes in these variables (Figure 2.14).

Regarding the weighting functions and profiting from previous experiences for DC-DC [57] and DC-AC converters [58], the double sigmoid is showed to be the most suitable weighting function for this application. It is well known that the weighting functions summation must be equal to 1 while their range must be from 0 to 1 . Also, the parameters affecting the double sigmoid (E 2.18) depends on the slope (parameter $\mathbf{a}$ in the equation) and the point of the inflexion (parameter $\mathbf{c}$ in the equation).

$$
F(x)=\left[\frac{1}{1+e^{-a(x-c)}}\right]
$$

\section{Modelling Procedure}

The modelling procedure of small-signal model is described adequate in this Chapter 2.2.2 section. This mechanism is reproduced as many times as different combinations of the four input variables $v_{d}, v_{q}, I_{r e f}$, and $Q_{r e f}$ are potential operating points according to the operating conditions in the charger. This approach implies the identification of twelve transfer functions for each small-signal model, multiply by the number of operating points selected. This model's structure implies a high degree of accuracy, but also a high complexity.

On the other hand, if we assume $v_{d}, v_{q}$, and $Q_{r e f}$ constant, then the number of transfer function in each small-signal model is limited to three and the operating points, either charging or discharging, are limited to a handy number. This simplified structure has been used during simulation and experimental results. The polytopic model has shown a good ability to represent the behaviour of the charger, even in largesignal operation.

This polytopic model based on the black-box small-signal model described before is a contribution of this thesis.

\subsection{Conclusions}

EV battery chargers has been reviewed describing the different configurations present in industrial applications. These configurations come to show partly the variety of EV battery chargers that exist currently in the market. The number of EVs is increasing gradually and due to their multiple schemes, it can give a 'headache' to be determined on their operations the 'right' time -exchange energy instantly- in a period of a day. Therefore, a model of determination has to be chosen. The second part of the chapter illustrates the modelling classifications for power converters starting with DC/DC converters and following with AC extension of single and three-phase systems. The classification also considers linear and nonlinear structures. 
In this thesis, a black-box identification method will be used using linear and non-linear methods. In the linear case, easier and more straight forward solutions have been used broadly, but in case of nonlinearities the system of identification is getting more complex. As an example, a significant change in the set point of the battery current in the circuit it might cause nonlinearities changing the dynamic of the system identification and the accuracy to be compromised. A technique to solve this problem is the polytopic model. This model it consists a summing of linear model responses in different operating points regaining the system's accuracy.

This thesis deals with the modelling of AC/DC battery chargers by identifying the transfer functions in any operating condition without knowing the specific details inside those chargers. This approach is called black-box modelling and the strategy is based on the information provided by the input and output variables of the charger. The research illustrates the flexibility of the model to work with single-phase and three-phase AC/DC chargers which are linked to on-board and off-board charging stations. An extensive set of tests is shown in the following chapters, verifying the accuracy of the proposed models in a wide variety of operating conditions. 



\section{CHAPTER}

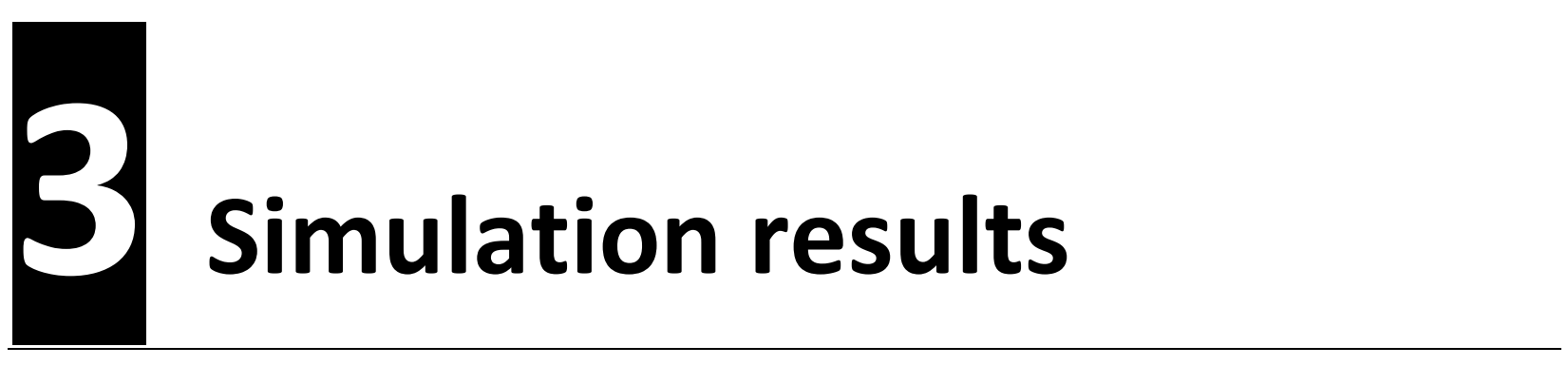

\subsection{Introduction}

In the previous chapter the state of the art of battery chargers modelling has been reviewed. In addition to that, a small-signal black-box behavioural model and a large-signal black-box behavioural model for battery chargers have been proposed. These models can represent the behaviour of a device accurately without any insight information, only by collecting the input and output signals. This black-box modelling strategy is the main contribution of this thesis.

In this chapter, the accuracy of the black-box models is verified through simulations. A single-phase bidirectional AC/DC converter is modelled in detail using the PSIM simulator, assuming that this switching model is an accurate representation of the actual battery charger. Then, the switching model works as a virtual prototype of the battery charger. This virtual prototyping technique simplify a lot the verification of the proposed behavioural black-box models and it assumes that switching models in modern simulators are an accurate representation of the actual converters. Then, the tests required to identify the model transfer functions are applied to the switching model instead of the actual converter. Once the model is generated, it is possible to compare the differences in performance between the switching model developed in PSIM and the black-box models proposed in this thesis.

As it was explained in chapter 2, using the information provided by the input and the output variables in the switching model, the transfer functions of the behavioural model are derived using MATLAB, and the black-box model is generated. To check the accuracy, the responses of the black-box model and the switching model are compared in different conditions, showing a very similar dynamic behaviour.

Initially the current chapter is creating a white switching model of a bidirectional battery charger in PSIM software. The charger which is chosen is 2-stage structure which considers a boost rectifier followed by a dual active bridge (DAB) dc-dc converter and a battery (Figure 3.1). 


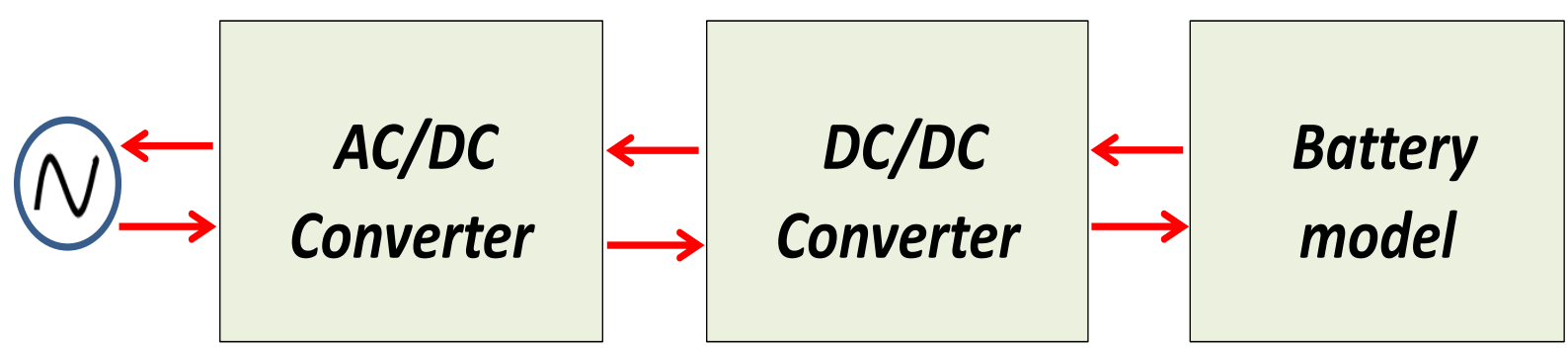

Figure 3.1 Battery charger's block diagram

The circuit will be decomposed and explained extensively along with the control design of each of the converters. The circuit has been selected showing the main characteristics of the commercial battery chargers and its bidirectional behaviour is particularly interesting to test the performance of the proposed black-box model in case of the most flexible architectures. It is important to remark that the design in not necessarily optimum because the purpose of the simulation in this chapter is the verification of the blackbox model accuracy compared with a white-box switching model and not, the optimization in the design of the bidirectional battery charger.

In the second part of the chapter a small-scale system level of battery chargers is shown. The bidirectional battery charger of the first part, a 2-stage including an AC/DC and a DC/DC converter is connected with another (identical to the first) DC/DC converter in parallel of the first DC/DC converter. Both of the DC/DC converters are followed by a battery. The black-box models show in this complex structure an excellent accuracy to anticipate the system behaviour.

\subsection{Boost rectifier}

In the current section a step-by-step creation of bidirectional battery charger will be shown along with the controllers in the PSIM program. As it was mentioned before the charger will be divided in three parts, in an AC/DC converter, a DC/DC converter and a battery. The battery charger is in single phase to indicate the charging point of a normal household.

The first part of this battery charger is a single-phase full fridge boost converter (figure 3.2). The circuit of this converter consists of 4 MOSFET's creating the full bridge. These switches combining power MOSFETs and their corresponding body diodes are capable to operate bidirectionally.

Assuming certain design values for the input and output voltages, the input current ripple, the output voltage ripple and the switching frequency, the main parameters of the converter (figure 3.3) are calculated, showing the values indicated in Table 3.1. 


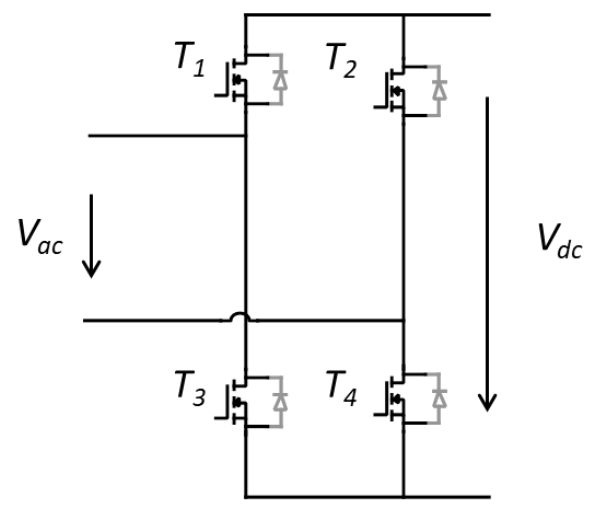

Figure 3.2 AC and DC Voltage across MOSFET's full bridge

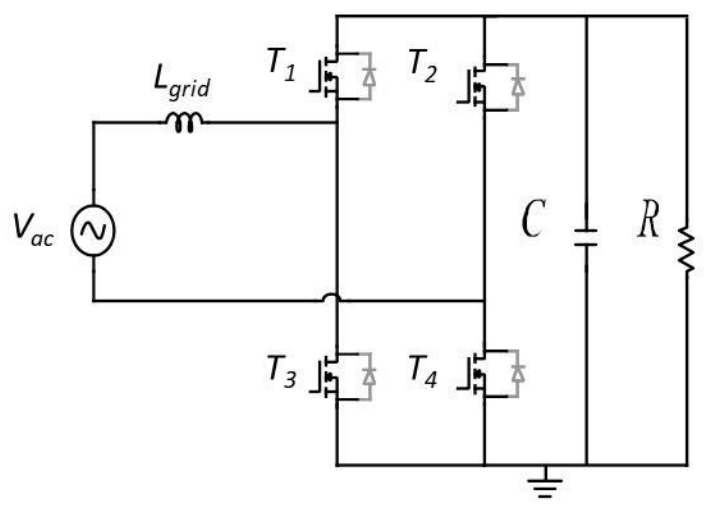

Figure 3.3 Complete boost rectifier circuit

\begin{tabular}{|c|c|c|}
\hline Symbol & Parameter & Value \\
\hline Pout & Rated power output & $4 \mathrm{~kW}$ \\
\hline Vac & Grid voltage & $325,27 \mathrm{~V}_{\mathrm{pp}}$ \\
\hline Lgrid & Boost inductor & $3 \mathrm{mH}$ \\
\hline $\boldsymbol{C}$ & Filtering capacitor & $10 \mathrm{mF}$ \\
\hline fswitching & Switching frequency & $50 \mathrm{kHz}$ \\
\hline
\end{tabular}

Table 3.1 Boost rectifier parameter values 


\subsubsection{Boost Rectifier Controller}

The design of the boost rectifier controller derives from the instantaneous reactive power theory. In a single-phase system, the grid voltage $\left(v_{\mathrm{g}}\right)$ can be transformed into an artificial system by leading the $\alpha$ phase with $90^{\circ}$ to the point of common coupling (PCC) of voltage and current, as it can be seen in the figure 3.4. The PCC voltage is considered to be held in the $\alpha$-axis whilst the $90^{\circ}$ leading voltage in $\beta$-axis in a hypothetical $\alpha-\beta$ plane. The signals produced after the addition of the $90^{\circ}$ lead are $v_{g}, v_{\beta}$ from the grid voltage. The input current can also be transformed in their corresponding values in the $\alpha-\beta$ plane, $i_{g}$ and $i_{8}$.

Those four signals with the help of the equations in (E 3.1) and (E 3.2) are used to calculate the instantaneous active $\left(p_{g}\right)$ and reactive power $\left(q_{g}\right)[77]$ :

$$
\begin{aligned}
& p_{g}=v_{g} i_{g}+v_{B} i_{B} \\
& q_{g}=v_{g} i_{B}-v_{B} i_{g}
\end{aligned}
$$

Reference active power $\left(p_{\text {ref }}\right)$ is calculated by taking into consideration the DC link voltage $\left(V_{d c}\right)$ of the boost rectifier and reference reactive power ( $\left.q_{\text {ref }}\right)$ the amplitude of the grid voltage $\left(v_{\mathrm{g}}\right)$. Later by using the currents $i_{a}$ and $i_{\beta}$ along with the equation in (E 3.3) the pulse width modulation (PWM) signals are produced for the four switches $d 1 \ldots 4$.

$$
\left(\begin{array}{c}
i_{\alpha} \\
i_{b}
\end{array}\right)=\left(\begin{array}{cc}
v_{\alpha} & v_{b} \\
-v_{B} & v_{\alpha}
\end{array}\right)^{-1}\left(\begin{array}{l}
p_{r e f} \\
q_{r e f}
\end{array}\right)
$$

According to the boost rectifier in figure 3.3 the voltage circuit expression which derives [78] is the following.

$$
\begin{aligned}
& v_{g}=L_{\text {grid }} \frac{d i \alpha}{d t} \\
& v_{B}=L_{\text {grid }} \frac{d i \beta}{d t}
\end{aligned}
$$

By applying $\omega=2 \pi f$ in the synchronous reference frame the $d$-q equation of voltage is calculated as it is shown below. 


$$
\begin{aligned}
& v_{d}=L_{\text {grid }} \frac{d i d}{d t}-\omega L_{\text {grid }} i_{q} \\
& v_{q}=L_{\text {grid }} \frac{d i q}{d t}+\omega L_{\text {grid }} i_{d}
\end{aligned}
$$

After deriving expressions (E 3.6) and (E 3.7) with the following expression:

$$
L_{\text {grid }} \frac{d}{d t}\left(\begin{array}{l}
i_{d} \\
i_{q}
\end{array}\right)=\left(\begin{array}{l}
V_{d} \\
V_{q}
\end{array}\right)+\left(\begin{array}{l}
V_{d c_{-} d} \\
V_{d c_{-} a}
\end{array}\right)^{\prime}-\left(\begin{array}{lr}
0 & \omega L \\
\omega L & 0
\end{array}\right)\left(\begin{array}{l}
i_{d} \\
i_{q}
\end{array}\right)
$$

The last part of of the (E 3.8) equation and specifically the term $\left(\begin{array}{lr}0 & \omega L \\ \omega L & 0\end{array}\right) \quad$ represents a coupling on the dynamics of the current. This means in case of controlling the $i_{d}$ and a change occurs it will affect directly the $i_{q}$ and the opposite. In order to remove this problem $\omega L_{\text {grid }} i_{d}$ and $\omega L_{\text {grid }} i_{q}$ are placed as decoupling terms in the controller.

In order to add reactive power compensation in the system active and reactive power has to be entered as references. Reference current values can be obtained by the following equation (E 3.9) which relates currents from the reference frame with active and reactive power.

$$
S=P+j Q=V I \cos \theta+j V I \sin \Theta
$$

By considering voltage in phase with the reference frame we know that, in such a way that $V_{q}=0$

$$
\begin{gathered}
V_{d}=V_{\text {max }} \\
V_{q}=0 \\
P=V_{\text {max }} I_{d} \\
Q=V_{\text {max }} I_{q}
\end{gathered}
$$

1- $\Phi$ vector representation with the equations shows in more detail how to obtain the reactive reference value:

$$
\mathrm{Q}=\mathrm{V}_{\mathrm{rms}} \mathrm{i}_{\mathrm{rms}} \sin \theta
$$




$$
\begin{aligned}
& Q=\frac{V \max }{\sqrt{2}} \frac{i m a x}{\sqrt{2}} \sin \theta \\
& Q=-\frac{V \max }{2} i_{q} \\
& i_{q}=-\frac{2 Q}{V \max }
\end{aligned}
$$

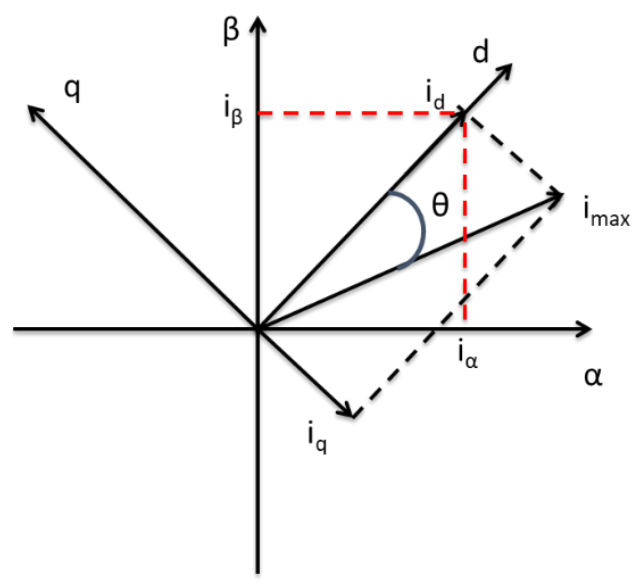

Figure 3.4 Creation of $\beta$-phase by $90^{\circ}$ phase shift of $\alpha$-phase

In case of single phase, Park transformation cannot operate immediately [79]. Therefor it needs first to reach a two-phase level by creating an orthogonal system with a $90^{\circ}$ phase shift added and creating $\beta$ phase. By using the vector representation above we can derive the equation below which transform AC variables $I_{\alpha}$ and $I_{\beta}$ to $I_{d}$ and $I_{q}$ DC values in the synchronous frame. This phase shift is shown in figure 3.5 as an all-pass filter box and inside the phase locked loop box after the $i_{a}$ input.

$$
\begin{gathered}
\left(\begin{array}{l}
i_{\alpha} \\
i_{\beta}
\end{array}\right)=\left(\begin{array}{l}
A \sin (\omega t+\theta) \\
A \sin (\omega t+\theta)
\end{array}\right) \\
T=\left(\begin{array}{ll}
\sin (\omega t) & \cos (\omega t) \\
-\cos (\omega t) & \sin (\omega t)
\end{array}\right) \\
\left(\begin{array}{l}
i_{d} \\
i_{q}
\end{array}\right)=\left(\begin{array}{l}
i_{\alpha} \\
i_{\beta}
\end{array}\right) T=\left(\begin{array}{c}
A \cos (\theta) \\
-A \sin (\theta)
\end{array}\right)
\end{gathered}
$$




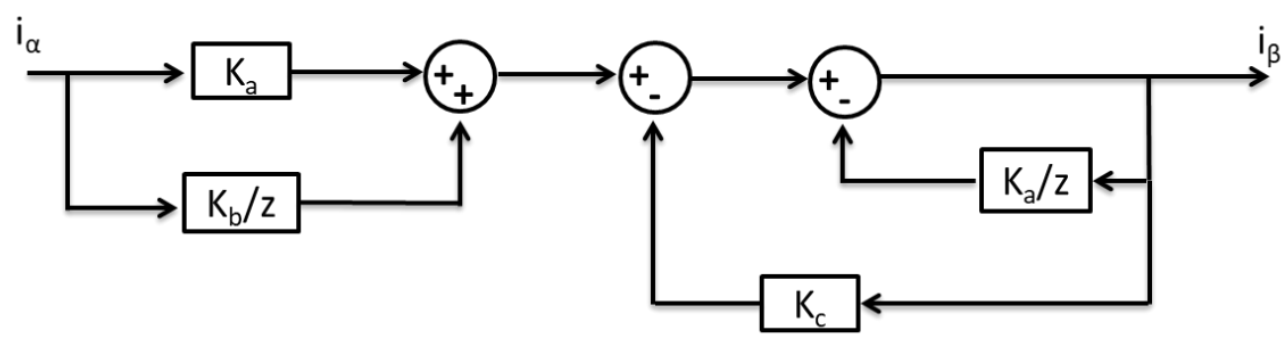

Figure 3.5 Digital form of APF controller

This shifting of the phase can be implemented with several techniques such as the phase delay block, the differentiator, and the all-pass filter. In this research all-pass filter (APF) is used because of several advantages it has over the differentiator and the phase delay block. All-pass filter is faster and not degrading the dynamic performance of the controller like on phase delay. Also is less noisy than the differentiator and is easy to implement.

Below the digital form of the APF controller is shown with the difference equations of the filter

$$
K_{a}=\frac{\omega T-2}{2}, \quad K_{b}=\frac{\omega T+2}{2}, K_{c}=\frac{\omega T}{2}
$$

where $\omega$ the angular frequency, T as sampling time in our system is $20 \mu$ s and frequency is $50 \mathrm{~Hz}$.

Then by deriving the equation of the controller in (E 3.18) we end up with the following expression

$$
\begin{gathered}
\left(K_{a}+\frac{K b}{z}\right) \mathrm{i}_{\alpha}=\mathrm{i}_{\beta}\left(1+K_{c}+\frac{K a}{z}\right) \\
\left(K_{a} \mathrm{z}+K_{b}\right) \mathrm{i}_{\alpha}=\mathrm{i}_{\beta}\left(\mathrm{z}+K_{C} \mathrm{z}+K_{a}\right) \\
i_{\beta}=\frac{K a z+K b}{(K c+1) z+K a}
\end{gathered}
$$

After obtaining the 2-phase system a $\Theta$ angle will be created with the aid of a phase locked loop (PLL). 2phase result will enter a $\alpha-\beta$ to $d$-q transformation. The $V_{q}$ output is multiplied by a proportional integral controller and then an angular frequency is added. The result is multiplied with an internal integrator ending up with a $\Theta$ angle. $\Theta$ angle is returning to feed the transformation box and thus the PLL is created as it is shown in figure 3.6. $\Theta$ angle also used in all the transformation boxes. The signals for the pulse width modulation (PWM) are extracted by controlling the $I_{d}$ and $I_{q}$ with DC link voltage $\left(V_{d c}\right)$ of the boost 


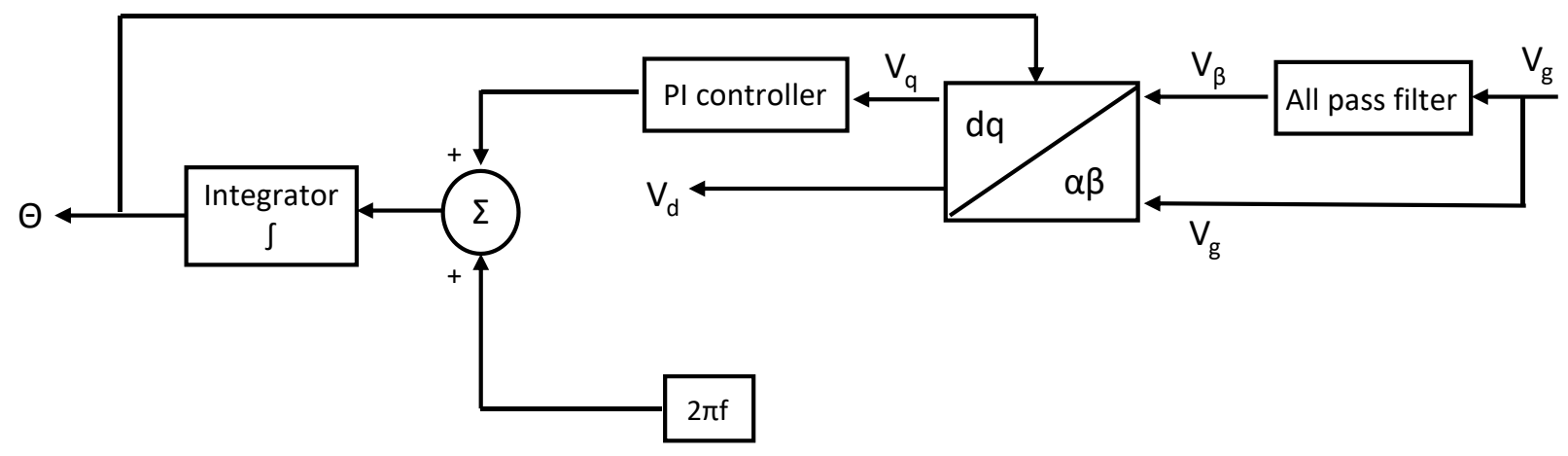

Figure $3.6 \Theta$ angle obtained by phase locked loop

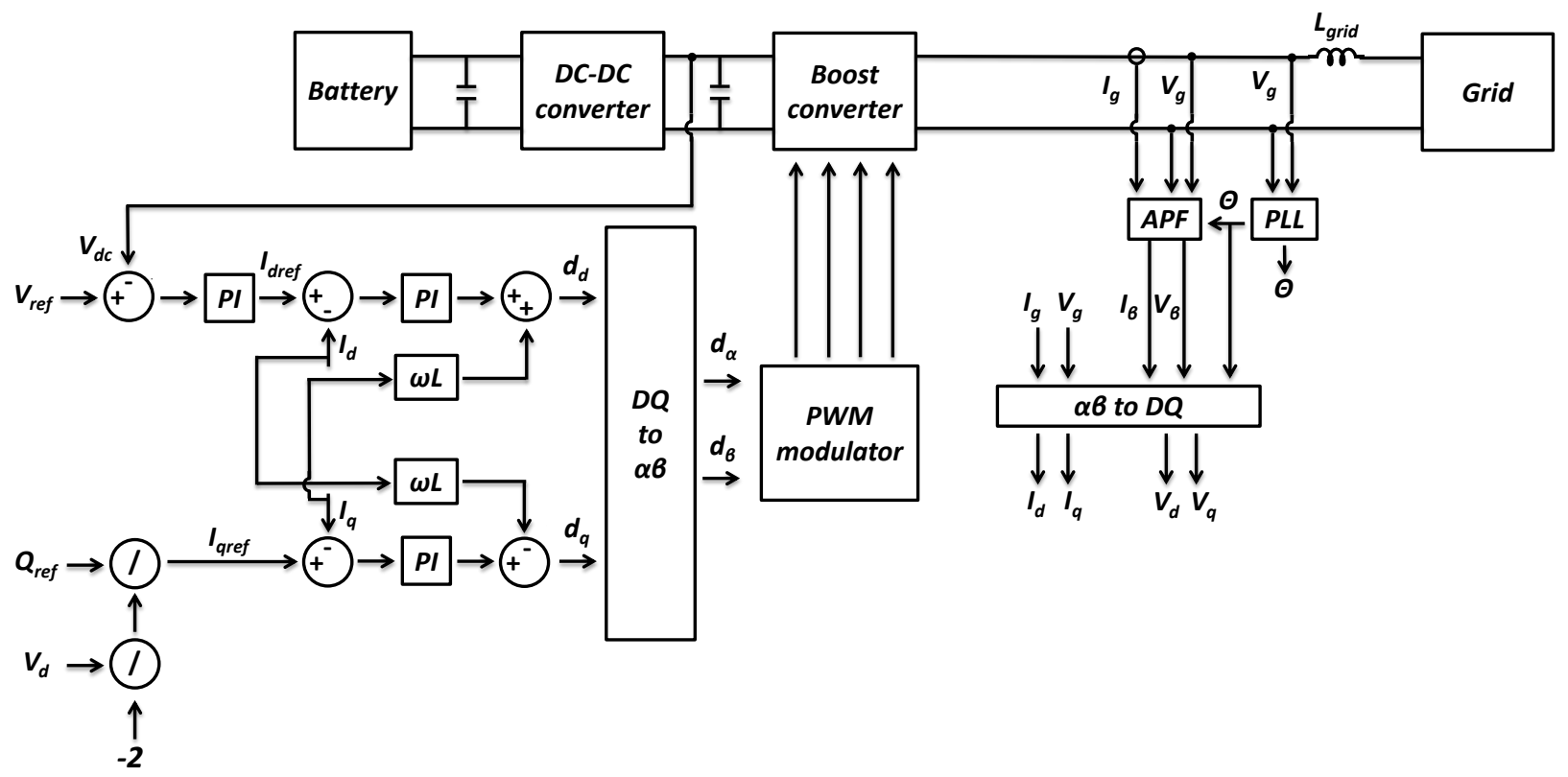

Figure 3.7 Full scheme of boost rectifier controller

rectifier and amplitude of the grid voltage $\left(\mathrm{v}_{\mathrm{g}}\right)$ respectively. Also, the reference current $\left(I_{\text {qref }}\right)$ has to maintain 0 for the power factor to retain unity.

Before the signals reach the PWM they pass through 3 proportional integrals (PI), 1 voltage control -after the DC link voltage- and 2 current controllers as it can be seen in figure 3.7 [79-81]. The PI's are used to ensure static error and fast rise time. The block diagram of both current controllers is shown below (figure 3.8). $I_{d r e f}$ and $I_{d}$ representing the input reference current and the output of the controller, respectively. Also, the $\alpha_{\mathrm{gi}}$ is representing the gain of the current sensor. Modulator and the voltage source converter shown in first order with $K_{d}$ gain and a $T_{d}$ average delay time associate for the sizing of the current controller. The $K_{d}$ gain equation is represented as $K_{d}=V_{\text {ref }} / V_{\max }$ and $V_{\max }$ as the maximum amplitude of the triangular carrier in pulse width modulation. PI controller $\left(\mathrm{C}_{\mathrm{i}}\right)$ [82] can represented as both 


$$
C_{i}(s)=K p_{i}+\frac{K i_{-} i}{s} \quad, \quad C_{i}(s)=\frac{s T z+1}{s T p}
$$

Assuming in (E 3.20) that PI compensator is cancelling the pole deducted from grid filter, $T_{z}$ it is considered to be equal with the $L_{\text {grid }} / R$, where $L_{\text {grid }}$ the value of filtering inductor and $R$ the resistance between filtering inductor and grid. From the figure 3.8, it can be derived the closed loop of the controller in (E 3.21)

$$
\frac{i_{d}(s)}{i_{\text {dref }}(s)}=\frac{a_{g i}+\frac{K_{d}}{T_{d} T_{p} R}}{s^{2}+s \frac{1}{T_{d}}+a_{g i} \frac{K_{d}}{T_{d} T_{p} R}}
$$

Later by putting together the denominators of ( $E$ 3.21) and the one from the $2^{\text {nd }}$ order transfer function written in canonical manner (E 3.22) $T_{p}, K_{p_{-} i}=T_{z} / T_{p}$ and $K_{i_{-} i}=1 / T_{p}$ are obtained (E 3.23). It is assumed that damping factor $\xi$ is equal to $\frac{\sqrt{2}}{2}$ [83].

$$
\begin{gathered}
G(s)=\frac{\omega^{2}}{s^{2}+2 \xi \omega s+\omega^{2}} \\
T_{p}=\frac{2 T_{d} K_{d} a_{g i}}{R}
\end{gathered}
$$

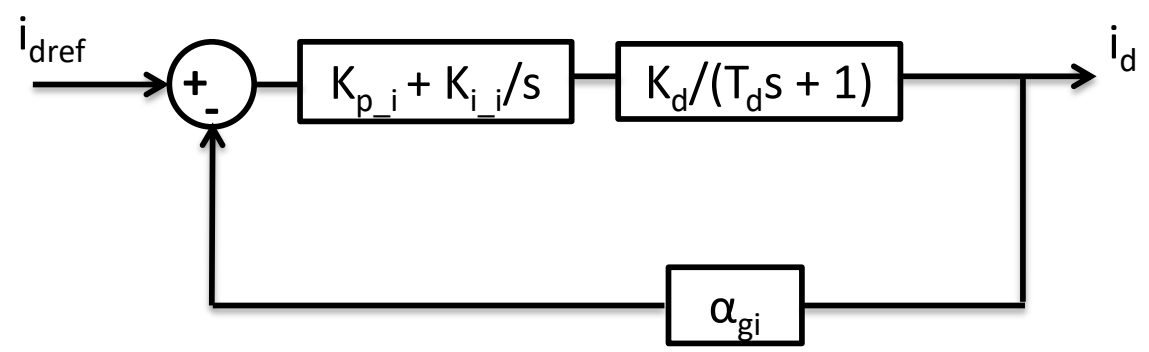

Figure 3.8 Block diagram of the internal current regulator 


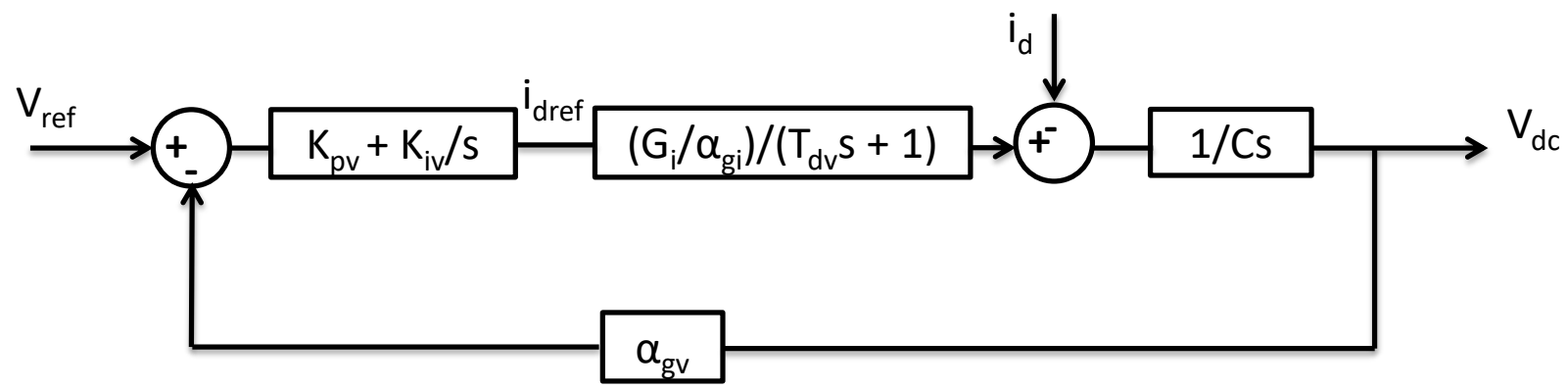

Figure 3.9 Block diagram of the voltage regulator

A more secure operation of the circuit is to control the DC capacitor voltage through a voltage regulator. The block diagam of the voltage regulator is shown in figure 3.9 , with reference voltage $V_{\text {ref }}$ and output voltage $V_{d c}$. The current controller is presented as $\left(G_{i} / \alpha_{g i}\right) /\left(T_{d v} S+1\right)$. The gain of current controller $\left(G_{i}\right)$ derived to $V_{g} / 2 V_{\text {ref }}$ from $V_{g} I_{g} / 2=V_{\text {ref }} I_{\text {ref }}$ with $V_{g}$ the voltage amplitude of the grid.

Following the figure 3.9 as in current controller we conclude with the closed loop in (E 3.24)

$$
\frac{\operatorname{Vdc}(s)}{\operatorname{Vref}(s)}=\frac{\frac{a_{g v} G_{i}}{a_{g i}} \frac{K_{p v}+K_{i v}}{T_{d v} C}}{s^{3}+s^{2} \frac{1}{T_{d v}}+s \frac{a_{g v} G_{i} K p v}{a_{g i}{ }^{T} d v}+\frac{a_{g v} G_{i} K_{i v}}{a_{g i}{ }^{T} d v} C}
$$

In the same manner as in current controller the denominator is compared with the canonical from this time of a $3^{\text {rd }}$ order polynominal (E 3.25) compensator gains are calculated in (E 3.26).

$$
\begin{gathered}
s^{3}+1.75 \omega \mathrm{s}^{2}+2.15 \omega^{2} \mathrm{~s}+\omega^{3} \\
K_{p v}=\frac{2.15 C a_{g i}}{1.75^{2} a_{g v} G_{i} T_{d v}} \quad, \quad K_{i v}=\frac{C a_{g i}}{1.75^{3} a_{g v} G_{i} T_{d v}{ }^{2}}
\end{gathered}
$$




\subsection{DC-DC converter}

The second part of the battery charger is a dual active bridge (DAB) (figure 3.10). A DAB is a bidirectional dc-dc converter composed of 2 active bridges communicating through a high frequency transformer [8486]. Both of the active bridges include 4 MOSFETs ( $T_{A 1}, T_{A 2}, T_{A 3}, T_{A 4}, T_{B 1}, T_{B 2}, T_{B 3}$ and $T_{B 4}$ ) each with their parasitic diode. The design of the converter simply includes a leakage inductance $\left(L_{k}\right)$ in series with the transformer. The transformer can be used for a high conversion ratio and assuring galvanic isolation as in this occasion.

The circuit symmetry structure of the DAB makes easier the bidirectional power flow through the circuit. Also, the low number of passive elements providing with evenly split of the current between the two sides of the converter.

The working principle of this topology is based on the phase shift $(\phi)$ strategy. The phase shift specifies the amount of power transferring in the converter and the direction of the current and is indicating on the equation of power transfer in (E 3.27) and in the figure 3.11 of a simplified DAB schematic. P as the power flowing from or to the output, $V_{1 r m s}$ and $V_{2 r m s}$ as the primary and secondary phase-shift voltage signals and $f$ as switching frequency.

$$
P=\frac{V 1 \mathrm{rms} V 2 r m s \sin \varphi}{2 \pi f L k}
$$

The phase shift is achieved by switching each full bridge with complementary pulse width modulated signals of $50 \%$ duty cycle and by $180^{\circ}$ the diagonal legs of each full bridge. This switching with the addition of the leakage inductance and the controlling of the switches of both bridges allow the signals properly phase shifting and regulating the power flow.

The difference between the phase shifting switching is shown in more detail in figure 3.12. The 4 upper figures are indicating the voltage of the MOSFET switches of the DAB and the last figure the current of the inductance. In the 2 upper figures it can observed the difference between the active bridge switches of the primary bridge (left side of the transformer). $\mathrm{T}_{\mathrm{A} 1}$ and $\mathrm{T}_{\mathrm{A} 4}$ diagonal switches are adapting while $\mathrm{T}_{\mathrm{A} 2}$ and $\mathrm{T}_{\mathrm{A} 3}$ have $180^{\circ}$ in difference because of the complementary pulse. The $3^{\text {rd }}$ and $4^{\text {th }}$ figures are shown the first 2 diagonal switches of each bridge from the secondary active bridge (right side of the transformer). $T_{B 1}$ and $T_{B 4}$ diagonal switches are adapting like $T_{A 1}$ and $T_{A 4}$ diagonal switches with only differernce a small phase shift $\phi$ in between them. This phase shift is indicating in the last figure of current inductance the transient time between the minimum and maximum current. 


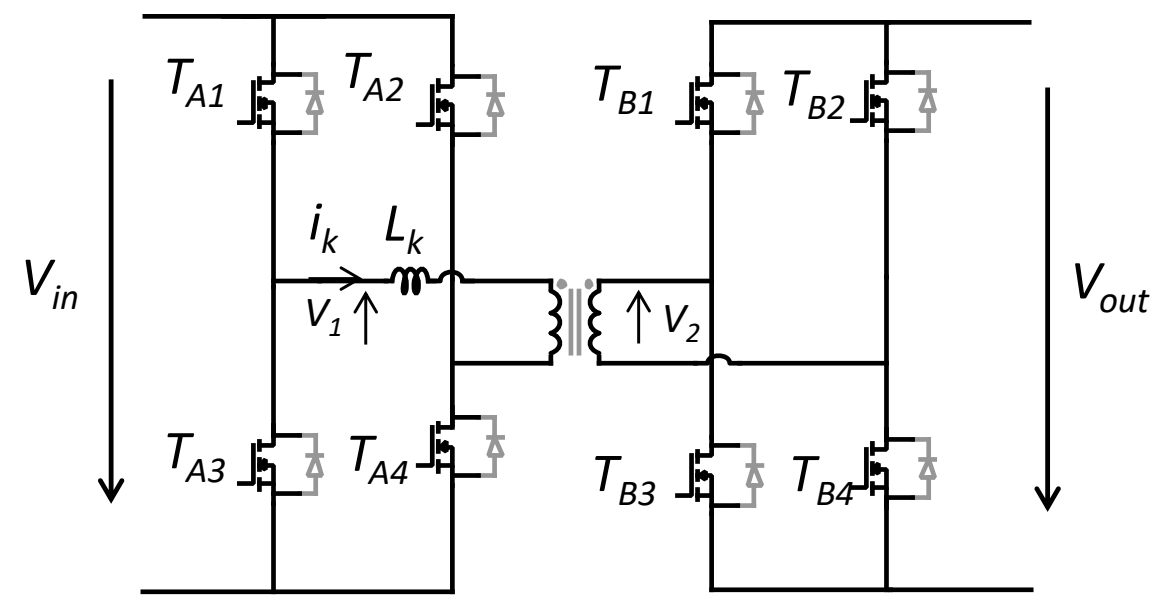

Figure 3.10 Classical DAB scheme

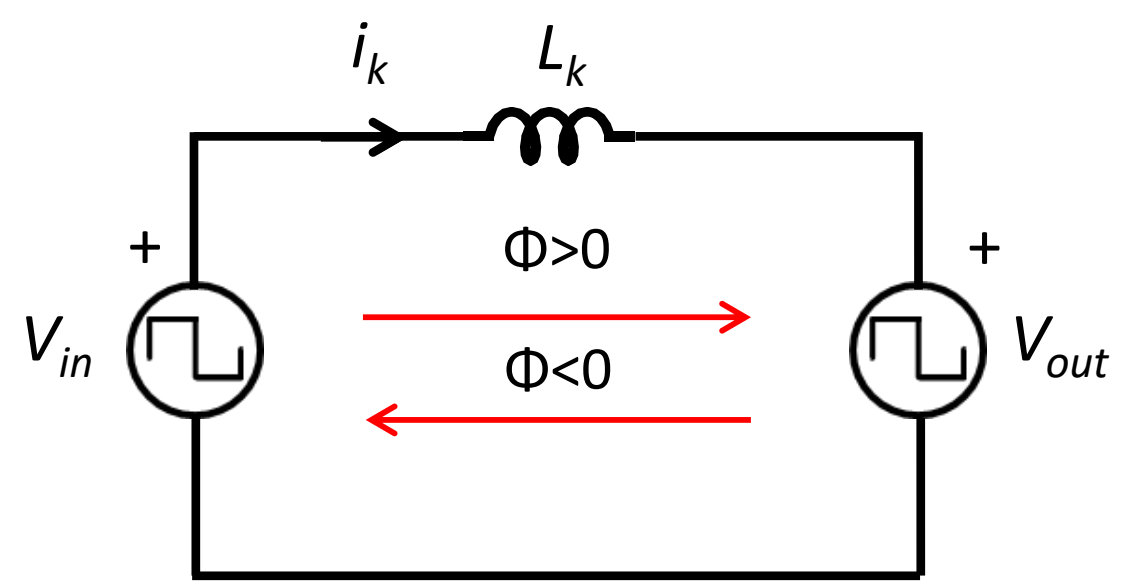

Figure 3.11 Simplified DAB scheme operation

By calculating the phase shift $\phi(1 / 20$ of a period) from the figure 3.12 and rearranging the power equation in (E 3.27) and we get the leakage inductance $\left(L_{k}\right)$ in (E 3.28)

$$
L_{k}=\frac{V_{1 r m s} V_{2 r m s} \sin \varphi}{2 \pi f P}
$$



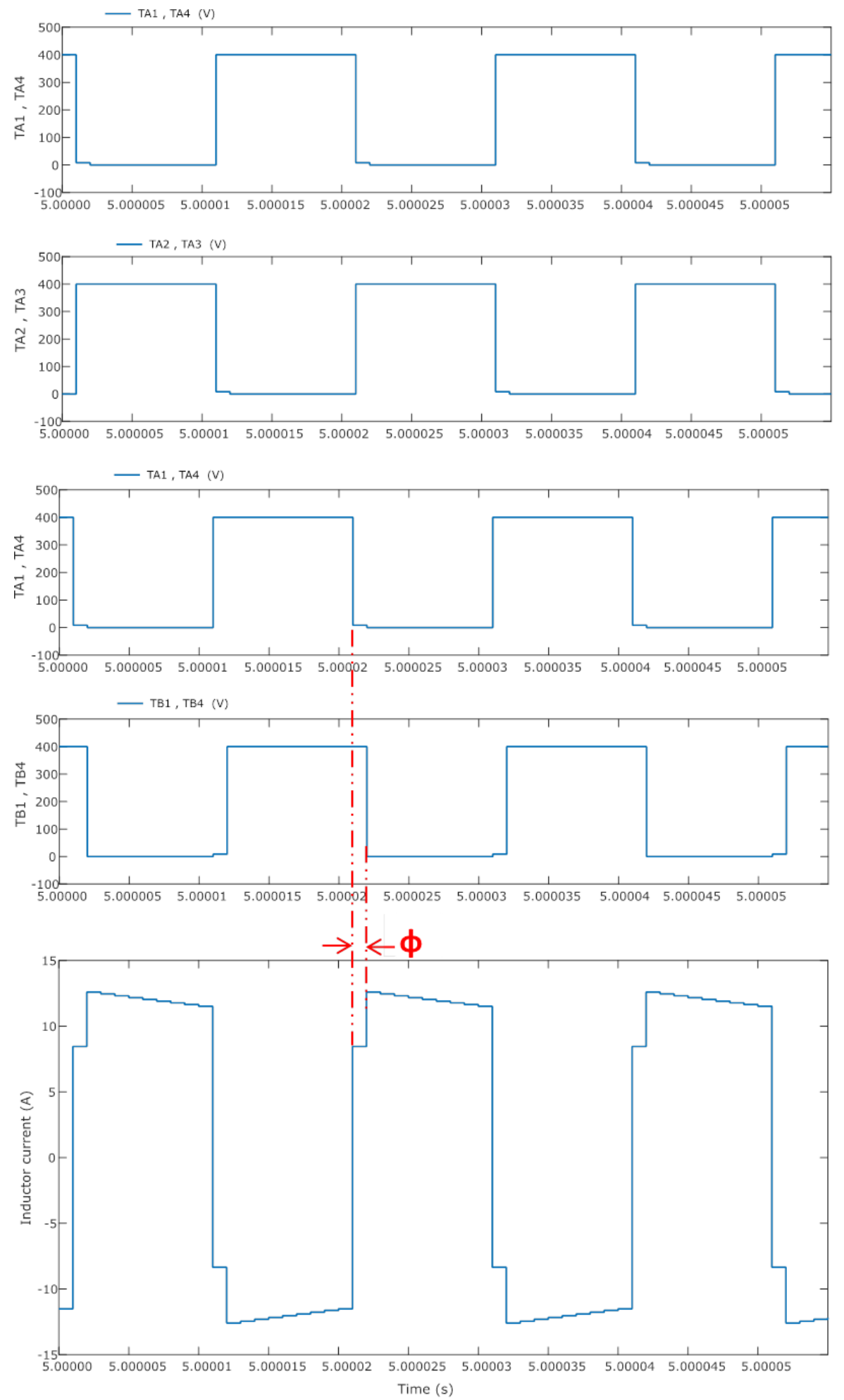

Figure 3.12 Obtaining phase shifting between the two parts of DAB 


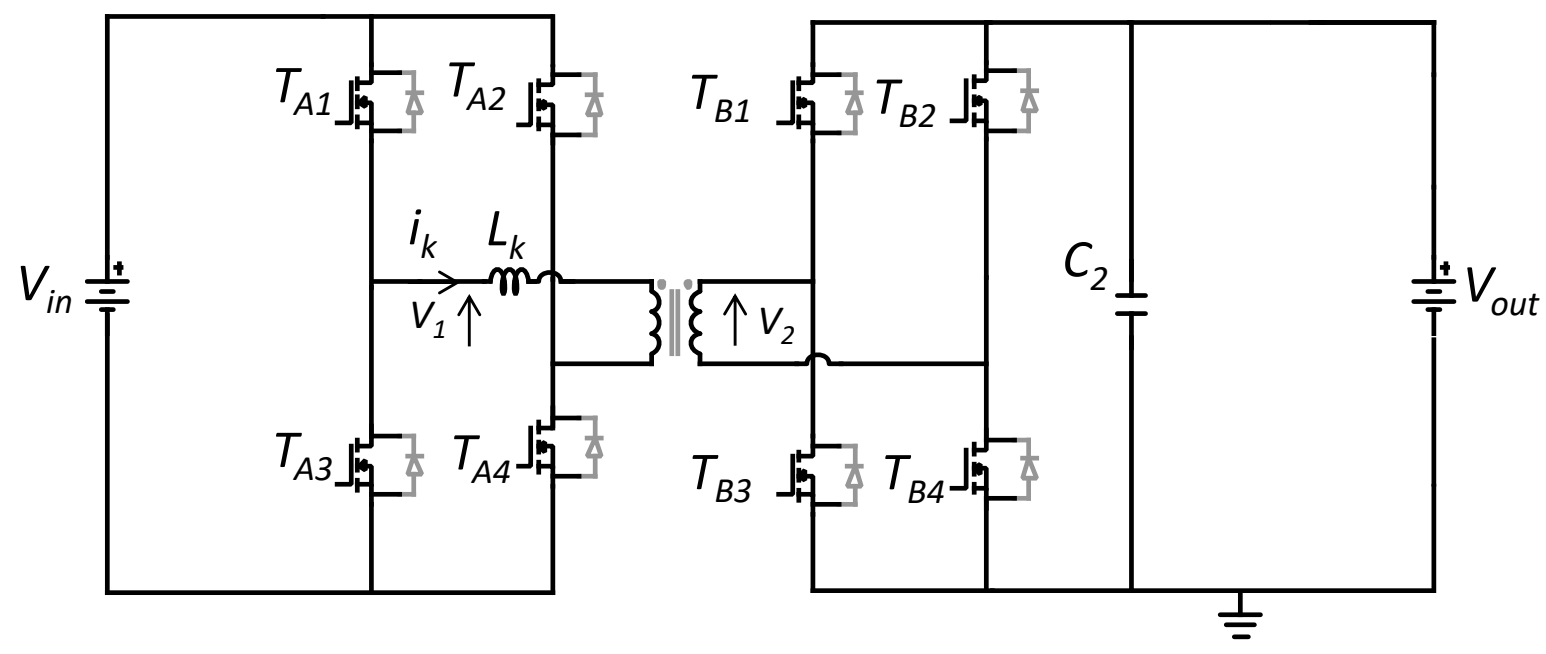

Figure 3.13 Full scheme of DC-DC converter

\begin{tabular}{|c|c|c|}
\hline Symbol & Parameter & Value \\
\hline $\boldsymbol{P}$ & Rated power output & $4 \mathrm{~kW}$ \\
\hline Vin & Input voltage & $400 \mathrm{~V}$ \\
\hline Vout & Output voltage & $400 \mathrm{~V}$ \\
\hline $\boldsymbol{L} \boldsymbol{C}$ & Leakage inductor & $40 \mathrm{uH}$ \\
\hline $\boldsymbol{\varphi}$ & Filtering capacitor & $0.34 \mathrm{mF}$ \\
\hline $\boldsymbol{f}$ switching & Phase shift & $18^{\circ}$ \\
\hline & Switching frequency & $50 \mathrm{kHz}$ \\
\hline
\end{tabular}

Table 3.2 DC-DC converter parameter values

The capacitor $\left(C_{2}\right)$ and last component of the converter as shown in figure 3.13. The parameter values of the DC-DC converter is shown below in the table 3.2. 


\subsubsection{DC-DC converter controller}

The controller of the DC-DC converter is simpler in comparison with the boost rectifier. This controller it consists of one proportional integral compensator for voltage and one integral compensator for the current of the battery. After the PI controllers and before the pulse width modulation a phase shift $(\phi)$ is depicted between the primary and the secondary switches. This phase shift is achevied through mathematical expressions from PSIM. The primary switches are muliplied by a parameter of sinx while the secondary by $\sin (\times 1-(x 2 *(\pi / 180)))$.

Similar to the boost controller the block diagrams of the controllers are cited along with expression in order for the gains to be calculated. In the current controller block diagram shown in figure 3.14, $\alpha_{i}$ is identified as the current sensor gain. Phase shift $(\phi)$ between the primary voltage $V_{1 r m s}$ and secondary voltage $V_{2 r m s}$ give the opportunity to control the power flow from and to the battery. By rearranging the equation (E 3.28) the gain is determined in (E 3.29).

$$
K_{D}=\frac{V_{1 r m s} V_{2 r m s}}{2 \pi f L_{k} I_{D C_{-} \text {out }}}
$$

Also regarding figure 3.14 the close loop is obtained in (E 3.30), placing $I_{D C}$ out and $I_{D C}$ ref after the $C_{2}$

$$
\frac{I_{D C_{\_} \text {out }}(s)}{I_{D C_{-} r e f}(s)}=\frac{a_{i} \frac{K_{i} K_{D}}{T_{d \_} i}}{s^{2}+s \frac{1}{T_{d} i}+a_{i} \frac{K_{i} K_{D}}{T_{d_{-} i}}}
$$

Comparing the denominator of second order transfer as in boost controller with the denominator in (E 3.30), the integral gain is shown in (E 3.31).

$$
K_{i}=\frac{1}{2 T_{d_{-} i} K_{D} \alpha_{i}}
$$

This voltage controller is able to ensure that the maximum voltage will not be exceeded in the battery part and cause any damage to the system. In the figure 3.15 below the voltage controller with the internal current control is shown, where $\alpha_{g_{-} v}$ appears as the voltage sensor gain. In the (E 3.32) transfer function of current controlling system is used to size the voltage controller. 


$$
\frac{I_{D C_{\_} o u t}(s)}{I_{D C_{\_} r e f}(s)}=\frac{1}{\alpha_{i}} \frac{1}{\frac{1}{s T_{d \_} v^{+1}}}
$$

Also by having alike closed loop transfer function as in (E 3.26), the compensator gains are illustraded in (E 3.33).

$$
K_{p_{-} v}=\frac{2.15 C 2 a_{i}}{1.75^{2} a_{g_{-} v} T_{d_{-} v}} \quad, \quad K_{i v}=\frac{C 2 a_{i}}{1.75^{3} a_{g_{\_} v} G_{i} T_{d_{-} v}{ }^{2}}
$$

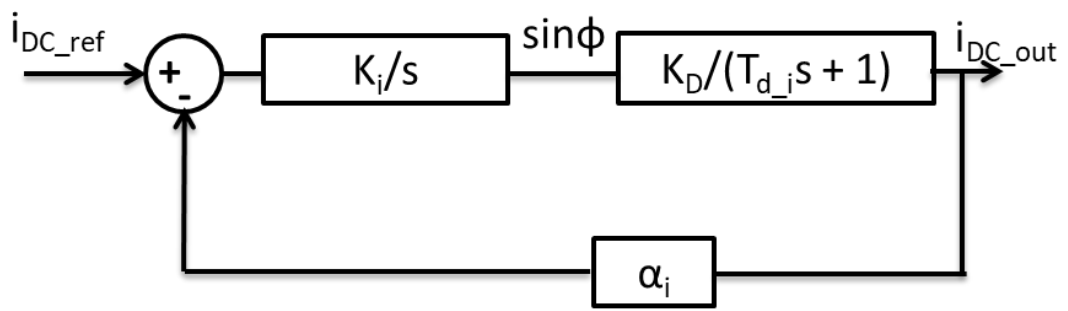

Figure 3.14 Current controller block diagram

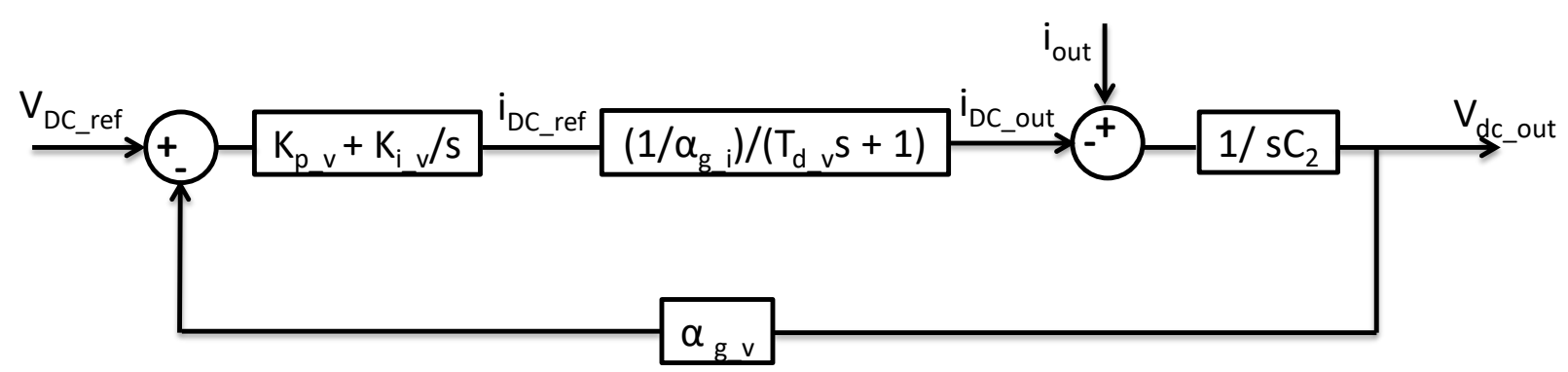

Figure 3.15 Voltage controller block diagram 


\subsection{Battery}

The third and last part of the converter is a model of a battery. In the previous section of DC-DC converter, the circuit was composed of 2 ideal voltage sources of equal voltage $400 \mathrm{~V}$ as input and output. In the current section the voltage source will be replaced by a battery model. The battery model will give a more realistic approach of a bidirectional battery charger.

The battery cell circuit proposed in figure 3.16 is of a Lithium-ion (Li-ion) through a Thevenin-based electrical equivalent curcuit. $V_{\text {in }}$ is considered as the open circuit voltage (OCV), $V_{\text {out }}$ the terminal voltage, $R_{0}$ the internal resistance, $R_{1 \text { to } N}$ the dynamic resistances and respectively the dynamic capacitances $C_{1}$ to $\mathrm{N}$ [87]. The resistances and the capacitors values of a battery model vary due to several factors such as the temperature, cycle life, current direction etc.

The example in [88] illustrates a battery cell model which consists the internal resistance and 2 RC branches. The internal resistance is representing the losses from the electrodes and the connectors, the first LC branch the effects on the surface of the electrodes and the second branch the diffusion procedure in the electrolyte. In a battery model the amount of RC brances are analogous to the desired accuracy. The normal amount of branches are from one to three.

The components which consist the battery cell can either be calculated or measured experimentally. An approach of experimentally collecting results is shown in [89] with a polymer Li-ion battery cell. The cell structure is the same as in [88] with the internal resistance $\left(R_{0}\right)$ the two RC brances dealing with the first

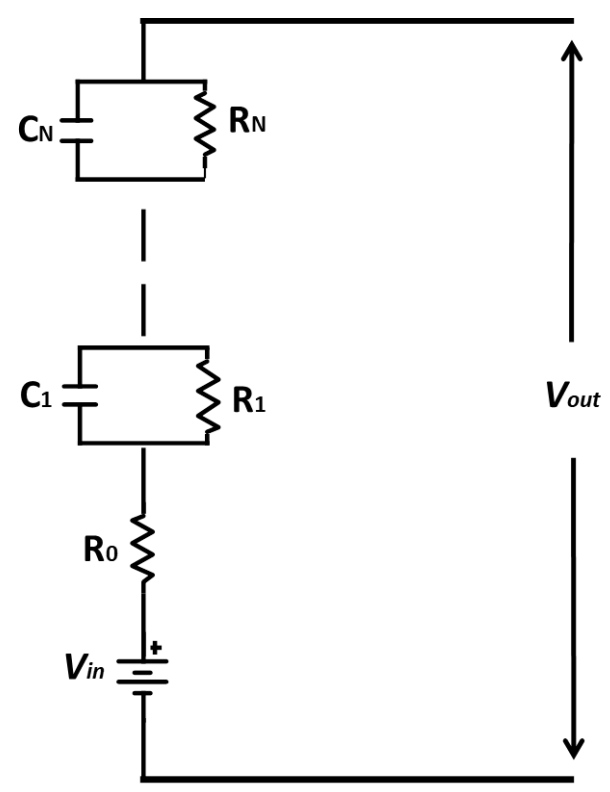

Figure 3.16 Battery cell circuit 
branch as short time ( $\left.R_{\text {short }}, C_{\text {short }}\right)$ and the second as long time $\left(R_{\text {long }}, C_{\text {long }}\right)$ transients. All components are functions of state of charge (SOC).

$$
\begin{aligned}
& R_{0}=0.1562 e^{-24.37 \mathrm{SOC}}+0.07446 \\
& R_{\text {short }}=0.3208 e^{-29.14 \mathrm{sOC}}+0.04669 \\
& C_{\text {short }}=752.9 e^{-13.51 \mathrm{soc}}+703.6 \\
& R_{\text {long }}=6.603 e^{-155.2 \mathrm{SOC}}+0.04984 \\
& C_{\text {long }}=-6056 e^{-27.12 \mathrm{soc}}+4475
\end{aligned}
$$

In the current case it is assumed that a battery pack of polymer Li-ion cells of $3.7 \mathrm{~V}$ and $800 \mathrm{mAh}$ is used to form the battery model. Assuming that the battery package operates at $400 \mathrm{~V}$ and $10 \mathrm{~A}$, a number of 108 cells formed in series and then in 12 parallel modules. This battery model consists only of one RC branch $\left(R_{1}, C_{1}\right)$ along with the internal resistance $\left(R_{0}\right)$ and the input voltage $\left(V_{i n}\right)$. This design selected because the accuracy of the software does not compromise by other factors. An example of this approach can be seen in the [90].

$C_{1}$ is reperesenting the total energy storage of the battery $[90,91]$ in the capacitors and is defined in the expression (E 3.39). Pout is defined as the output power of the battery package $4 \mathrm{~kW}, \mathrm{Vmin}$ and $\mathrm{Vmax}$ as the maximum and minimum output voltage, $410 \mathrm{~V}$ and $390 \mathrm{~V}$ respectively. The expression is calculated in kWh.

$$
C_{1}=\frac{2\left(3600 P_{\text {out }}\right)}{V_{\max }{ }^{2}-V_{\min ^{2}}}
$$

Therefor the value of $C_{1}$ is $1800 F, R_{0}$ has chosen to be $0,4 \Omega$ and $R_{1}$ as $4 \Omega$. $R_{1}$ has chosen to be significant larger than $\mathrm{R}_{0}$ in order to reduce the speed of discharge.

\subsubsection{Charging Technique}

Different techniques can be used to achieve battery charging [92,93,94]. Some methods are for fast charging, other more economical or simple to design and others safer for the equipment. One good example in terms of economy and simplicity of design is the overnight charger. This charger operates in a constant current mode with a trickle current, a small amount of current which need around 10 hours to full capacity. The drawback in this technique is the dependence of the user to manage the charging process, something that may lead to overcharging of the battery. 
A technique which is more in favour of fast charging is by using an increasing constant current (CC) charging. This technique it needs a more advance circuitry than the overnight charger which will terminate the charging when it reaches full capacity. This method provides with a fast charging ( 2 to 3 hours), but it gives some disadvantages in long-term usage. The constant high current charging causes different problems in the electrochemical process [95] and as a result the battery's cycle life reduced as well with its capacity.

A more known and most usable charging technique is a similar approach to the second approach. In this method a constant current is applied until the voltage rises in a pre-set point. At this point constant voltage (CV) mode will be on, with constant voltage held constant while the current will decrease gradually. As soon as the current reaches a minimum value it will stop charging. This approach of charging is used also in this section but without the last part of dropping the current in the last phase, as it can be seen in figure 3.17.

In the figure 3.18, the battery circuit explained in battery circuit section is shown. In parallel with the battery, it can be seen a current and a voltage source. The current source represents the reference current applied from the controller in order to achieve constant current mode while the $V_{\text {in }}$ switch is activated as soon as the voltage sensor reach the pre-set maximum voltage and turn the circuit in constant voltage mode. As it was mentioned, a maximum voltage has been set with a comparator on the end of battery circuit at $410 \mathrm{~V}$ and another comparator for low voltage limit at 390V. Those 2 limits will

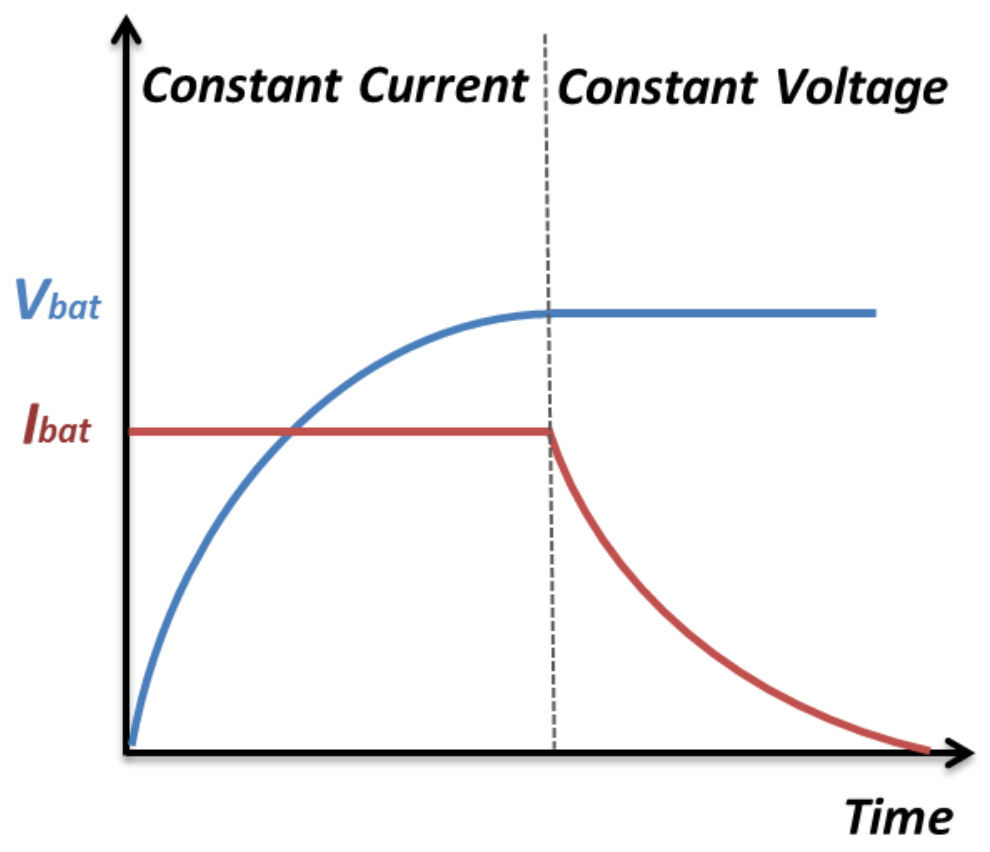

Figure 3.17 EV battery charging technique using CC/CV technique 
keep the battery from overcharging and depleting the battery respectively. A reference voltage $V_{b_{-} \text {ref }}$ is set slightly above the limit of the voltage comparator at $415 \mathrm{~V}$. The substaction with the masurement voltage $\mathrm{V}_{\mathrm{b} \_m e a s}$ from the voltage sensor will pass through the voltage PI controller calculated from the DCDC converter controller section. After the PI controller the switching part takes place, showing that the voltage is induced the circuit in case the voltage range of the battery is above $410 \mathrm{~V}$ and lower than $390 \mathrm{~V}$, having constant voltage. In the other hand if the voltage range in the battery is between 410V - 390V, reference current is induced, entering constant current mode. After the switches, the result is substacted from the meaured battery current $I_{b_{b} \text { meas }}$, placed between the battery and end of DC-DC converter (here after the $V_{\text {in }}$ switch). The last part is a current PI controller also calculated in DC-DC converter controller section and then a phase-shift $\phi$ is obtained (figure 3.19).

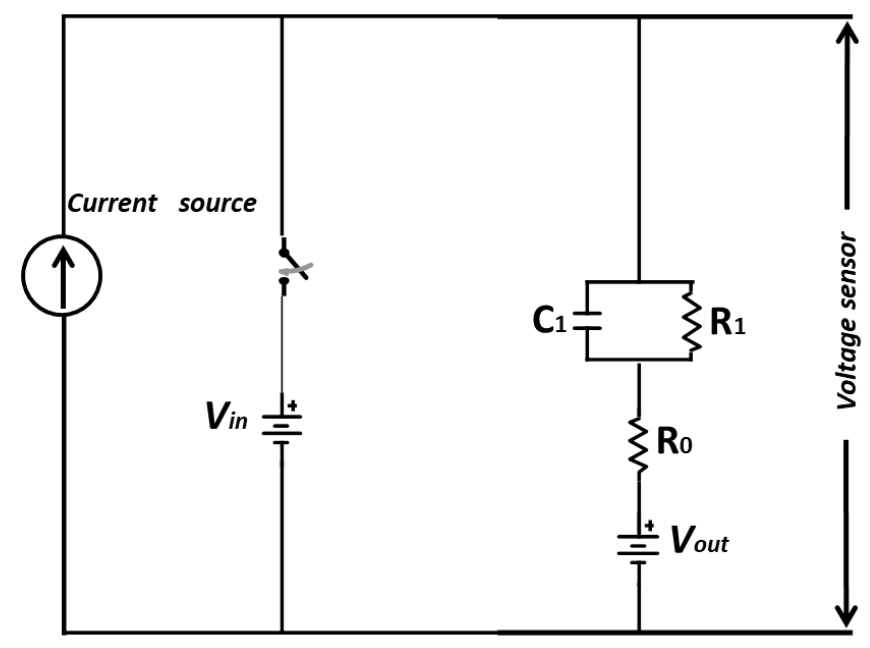

Figure 3.18 Battery circuit scheme
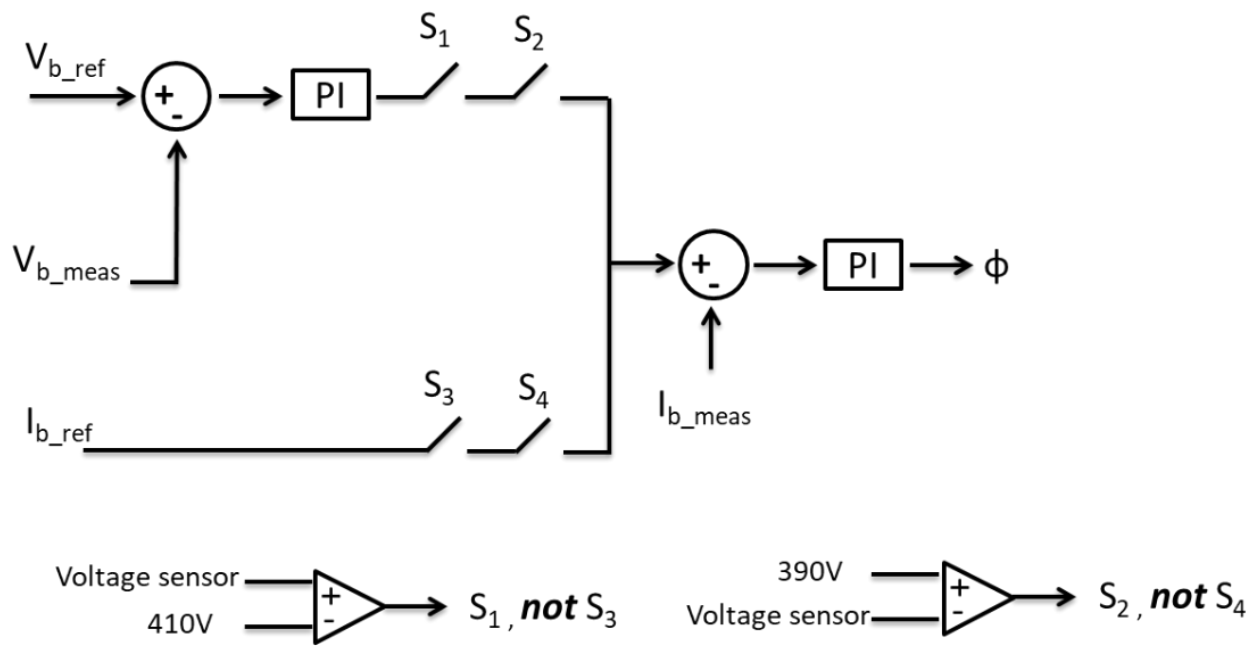

Figure 3.19 Controller of battery circuit 


\subsubsection{Soft charging}

After the completion of the bidirectional battery charger circuit, a start-up inrush current has been observed on the AC and DC part. A solution to supress this start-up inrush currents is a soft start circuit strategy [96]. A simple solution of a resistor in parallel with a time sharing switch has been applied in both parts. Initially both capacitors $C_{\text {and }} C_{1}$ charging through the resistors $R_{s s_{-} 1}$ and $R_{s s_{2} 2}$ that are limiting the inrush current. As soon as the capacitors are charged the switches are closing giving a low resistance way for higher current values. The switch on the $A C$ side $S_{\text {s__ } 1}$ is closing at 1,8 second which is parallel with $R_{s s_{1} 1}$ of $25 \Omega$ and $\mathrm{S}_{\mathrm{ss} \_}$is closing at 0.03 second which is parallel with $\mathrm{R}_{\mathrm{ss} \_}$of $10 \Omega$.

\subsection{Black-box model for linear simulation of a battery charger}

The three main circuit topologies of the charger -boost rectifier, DC-DC dual active bridge, battery circuithave been explained in detail in the terms of operation and giving adequate evidence of the values selected. Beside the circuits, the controller of the boost rectifier and the dc-dc converter explained along with the charging technique of the battery circuit.

This section will cover the transformation of the power simulation battery charger observed previously. The identification process as mentioned before in the literature review is the pathway to follow in order to achieve the black-box model. This battery charger is a bidirectional single phase with operating steps in both ends of the scheme.
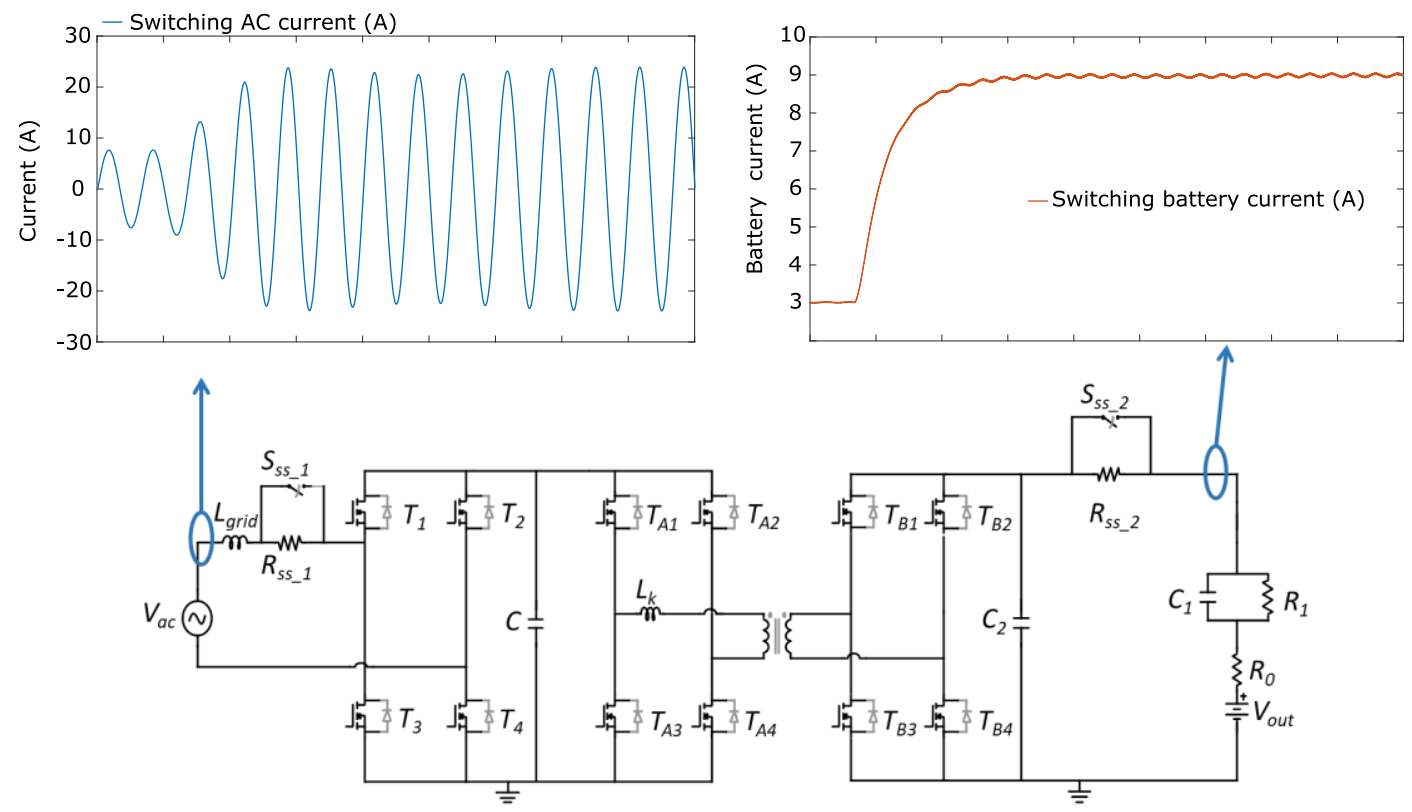

Figure 3.20 Switching single-phase bidirectional battery charger 
In the figure 3.20 the first part of the identification process is shown. The full scheme of the single-phase bidirectional battery charger is picked up. On the two ends it shows the data collection from the simulation circuit featuring the current step of $3 \mathrm{~A}$ to $9 \mathrm{~A}$ charging the battery. We must have in mind that these two points are the last visible points before entering the black-box.

A better view of a black-box model is illustrated in the figure 3.21. The current simulation battery charger is transformed into a block diagram of a black-box model. The input variables in the system are shown in the left side and the outputs on the right side of the black-box model. I Iref is the control reference current and the $Q_{\text {ref }}$ the control reference reactive power and $I_{\text {bat }}$ the battery output current. Except the dc variable an $A C$ variable is entering the black-box charger. The $V_{\alpha}$ variable is the single-phase $A C$ input voltage. As it is well known the black-box model is familiar with the DC input/output values. In case of AC values, a preparatory work has to be preceded. In case of our example an AC single phase is going through an orthogonal signal generator which is giving an identical signal with a $90^{\circ}$ phase shift, $\mathrm{V}_{\beta} . \mathrm{V}_{\alpha}$ and $\mathrm{V}_{\beta}$ are still in the $A C$ framework until a rotation from an $\alpha-\beta$ to $d$-q transformation box applies. Therefor by gaining the new values of $V_{d}$ and $V_{q}$ the part of input variables is complete. In the output part in order to depict the $A C$ value $I_{\alpha}$ from $I_{d}$ and $I_{q}$ a reverse rotation of the previous transformation box is needed, from $d-q$ to $\alpha-\beta$.

The input variables $\left(V_{d}, V_{q}, I_{\text {ref }}, Q_{\text {ref }}\right)$ are subtracted by the respective nominal values $\left(V_{d}{ }^{d c}, V_{q}{ }^{d c}, I_{\text {ref }}{ }^{d c}, Q_{\text {ref }}{ }^{d c}\right)$ giving a small signal perturbation of the input variables in the black-box model. On the other hand, the output nominal values $\left(I_{d}{ }^{d c}, I_{q}{ }^{d c}, I_{b a t}{ }^{d c}\right)$ are added to the small-signal perturbations. These small-signal perturbations are applied in one input variable per time observing the changes in each output variables. This procedure is useful to obtain the transfer functions of the model through identification algorithms. In a model like in figure 3.21 with 4 inputs and 3 outputs 12 transfer functions can be obtained. All perturbations in that figure are represented by $\hat{x}$ letters.

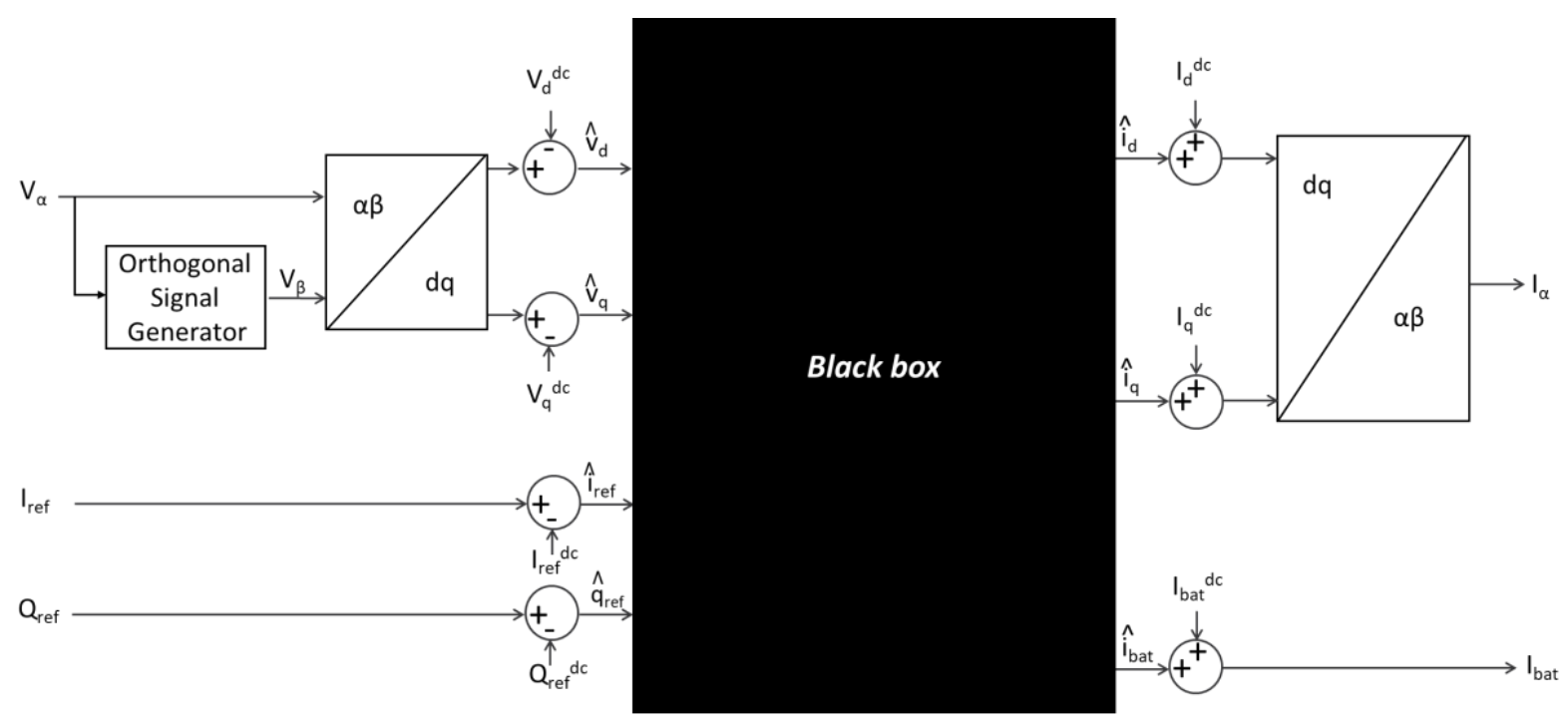

Figure 3.21 Block diagram for a black-box model of battery charger 
The matrix equation (E 3.40) shows the inputs and outputs of the black-box model in figure 3.21 along with 12 transfer functions.

$$
\left(\begin{array}{c}
\hat{i}_{d}(s) \\
\hat{i}_{q}(s) \\
\hat{i}_{\text {bat }}(s)
\end{array}\right)=\left(\begin{array}{cccc}
Y_{d d}(s) & Y_{d q}(s) & H_{d r e f}(s) & H_{d q}(s) \\
Y_{q d}(s) & Y_{q q}(s) & H_{\text {qref }}(s) & H_{q q}(s) \\
Y_{\text {batd }}(s) & Y_{\text {batq }}(s) & H_{\text {batref }}(s) & H_{\text {batq }}(s)
\end{array}\right) \cdot\left(\begin{array}{c}
\hat{v}_{d}(s) \\
\hat{v}_{q}(s) \\
\hat{i}_{r e f}(s) \\
\hat{q}_{r e f}(s)
\end{array}\right)
$$

The subindex in the left-hand side of the matrix it shows the outputs variables of the system while the right-hand side the input variables. $Y_{d d}(s), Y_{d q}(s), H_{d r e f}(s), H_{d q}(s), Y_{q d}(s), Y_{q q}(s), H_{\text {qref }}(s), H_{q q}(s), Y_{\text {batd }}(s), Y_{\text {batq }}(s)$, $\mathrm{H}_{\text {batref }}(\mathrm{s}), \quad \mathrm{H}_{\text {batq }}(\mathrm{s})$ are representing the dynamic response of the system. As an example, choosing the first transfer function $Y_{d d}(s)$, we can see the response on the $d$-component of the output current created by the input voltage $d$-component perturbation. At the same moment, the rest of the input kept constant. This explanation can be seen in the following mathematical expression (E 3.41). Likewise, the rest of the transfer functions have been created.

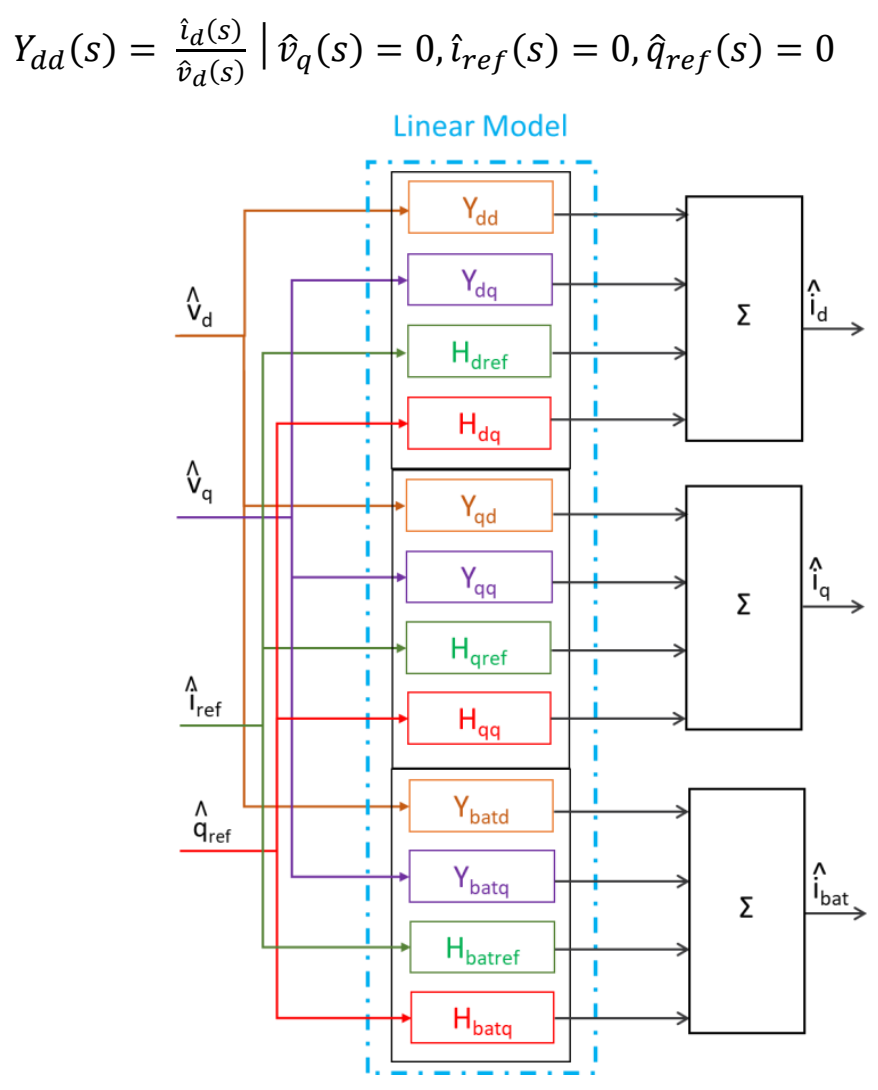

Figure 3.22 Identified transfer functions for battery charger 


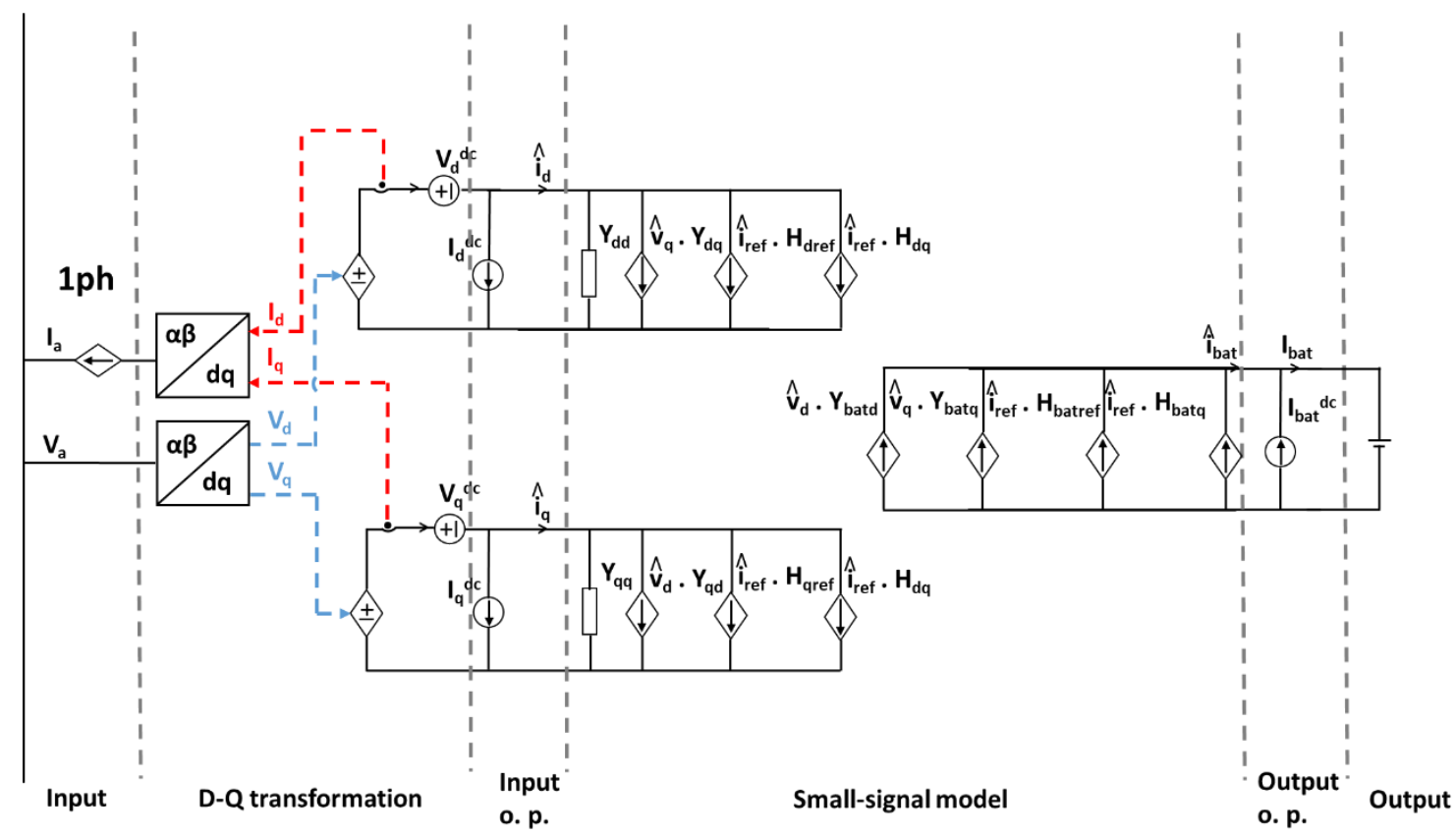

Figure 3.23 Equivalent circuit of the black-box model of battery charger

In figure 3.22 the transfer functions from (E 3.40) are shown below to fill the black-box model space of figure 3.21. The transfer functions have been estimated in a time domain with sample time $1 \mathrm{e}-6$ following the simulation (PSIM) model.

An equivalent circuit of the block diagram in figure 3.21 and the mathematical expression in (E 3.40) of the complete black-box model can be seen in the figure 3.23.

\subsubsection{Comparison of black-box and switching model}

After the estimation of the transfer functions -in this case from transfer function models, continuous timeand the implementation of the black-box model, the last step is to validate the linear models. The results from the first simulation charger are depicted and along with black-box model results their similarity will be illustrated. The 3 type of the tests implemented were from the reference control current, the reactive control power and the voltage amplitude control.

In each control, a perturbation equal to the step applies in the specific control in order to create the linear models. By keeping the rest of the inputs without any power steps (E 3.41) and with the response on the output variables the procedure for the linear model creation will be initiated. As an example, for the creation of the transfer function of the reactive power in test 1 , the control starts from 0 (in the $Q_{\text {ref }}$ input) at steady state and increases by $1 \mathrm{KVar}$. All captions have been taken in the same duration of time. 
The first type of tests applies in the boost rectifier, on the control of reference reactive power in the PSIM battery charger. The identification procedure from control reference current, creates through Matlab identification tools 3 transfer functions. The transfer functions created are marked in the figure 3.22 with red colour $\left(\mathrm{H}_{\mathrm{dq}}, \mathrm{H}_{\mathrm{qq}}, \mathrm{H}_{\mathrm{batq}}\right)$. The black-box model is using these linear transfer functions in the same tests to compare with the PSIM tests related to the changes from the reference control reactive power. In this test the current control will remain at $3 \mathrm{~A}$ in $\mathrm{G} 2 \mathrm{~V}$ mode.

In the figure 3.24 the first test of controlling the reactive power is shown. Initially no reactive power is applied in the charger and at a time of $0.05 \mathrm{~s}$ a step of $1 \mathrm{KV}$ ar is added in the input of the reactive power $\left(Q_{\text {ref }}\right)$ and the system is charging with inductive operation. The first two graphs of the figure 3.24 portrayed the black-box results in blue colour while in the last graph in blue and yellow. In the upper part of the graph is illustrating the change caused by the addition of the step in the AC current. The middle graph of the same figure it shows the phase-shifting of the AC current with respect of the AC Voltage. Lastly in the bottom of the graph, shows how the $d$-q components -in dc part- of the AC current are affected.

As in the previous example of controlling the reactive power, the identification process through Matlab identification tools will be used to create the respective transfer functions. The 3 transfer functions are indicated in figure 3.24 with brown colour $\left(\mathrm{Y}_{\mathrm{dd}}, \mathrm{Y}_{\mathrm{qd}}, \mathrm{Y}_{\text {batd }}\right)$. The black-box model will use these linear transfer functions in the same tests to compare with the PSIM tests related to the changes from the voltage amplitude.

The second type of tests applies on voltage amplitude through the Vd output of an $\alpha \beta / \mathrm{dq}$ transformation block in the PLL on the controller of the boost converter keeping the phase angle constant. Due to the boost rectifier design, the change in the voltage can be applied only if there is a value in the Qref input and therefor this leads to keep the 1KVar from the previous test. In the phase locked loop (PLL) figure 3.6 from the boost rectifier controller section, the $V_{d}$ will act as the input to create the linear model. The DC voltage of the $d$-q framework $\left(V_{d}\right)$ initially operates in $325 \mathrm{~V}$ and in a certain moment a step of $60 \mathrm{~V}$ is applied at $0.1 \mathrm{~s}$. The middle graph of figure 3.25 shows the change of AC current from the 60V step while in the upper the change of the AC current is shown in respect with the AC voltage. In bottom as in the previous figure 3.24 show the $d$-q components of the AC current. Also, in this caption all results are taken in the same duration of time.

The third type of test is dedicated in the dc-dc converter and applies on the control of reference dc current ( IDC_ref $\left._{\text {f }}\right)$ in PSIM at a certain time. All captions are also in the same time duration. After the identification process through identification tools in Matlab, the transfer functions caused by the change in the control reference current are shown in figure 3.22 with green colour $\left(\mathrm{H}_{\text {dref }}, \mathrm{H}_{\text {qref }}, \mathrm{H}_{\text {batref }}\right)$. The black-box model will use these linear transfer functions in the same tests to compare with the PSIM tests related to the changes from the reference control current.

The first test of the reference control current it operates in a G2V mode (figure 3.26). Initially the current is operating in a steady-state mode in $3 \mathrm{~A}$ and at $0.05 \mathrm{~s}$ a $6 \mathrm{~A}$ step is applied. The upper graph from the figure 3.26 is illustrating the $A C$ current response from the $6 A$ step. The following graph shows the $A C$ 
current response along with the $A C$ grid voltage and the $3^{\text {rd }}$ graph the changes in $d$ - $q$ components of the $\mathrm{AC}$ current. In the bottom graph of the figure 3.26 the change in the battery current is shown.

The second test from the reference control current is shown in figure 3.27 and operates in V2G mode. In this test the initial steady-state is in V2G at $-3 \mathrm{~A}$ indicating the opposite direction of the current. A negative step of $-3 \mathrm{~A}$ is applied at $0.1 \mathrm{~s}$ keeping the negative current value at $-6 \mathrm{~A}$. The upper graph shows the phase change of $A C$ current with $A C$ voltage by $180^{\circ}$ due to the opposite direction of the current. The below graph shows the change in the AC current from the current step and the following its $\mathrm{d}$-q components affect. In the bottom graph the battery current change is shown.

The last test on the reference control current it operates in both G2V and V2G modes (figure 3.28). It can be easily observed whether the current is induced to the battery ( $\mathrm{V}_{\mathrm{ac}}$ and $\mathrm{l}_{\mathrm{ac}}$ in phase) and whether current is sent back to the grid $\left(\mathrm{V}_{\mathrm{ac}}\right.$ and $\mathrm{l}_{\mathrm{ac}}$ phase shift by $\left.180^{\circ}\right)$. At start battery charger is operating in a $\mathrm{G} 2 \mathrm{~V}$ mode at a steady-state current of $3 \mathrm{~A}$. At $0.1 \mathrm{~s}$ a negative step of $6 \mathrm{~A}$ is applied on the controller causing the direction of the current to change and the battery charger operate as V2G. The graphs shown in 3.28 have the same pattern as in 3.27. The upper graphs with the combination of AC voltage and current, followed by the AC current graph and its d-q components and in the bottom graph the battery side current.

The last 3 transfer functions from figure 3.22 in purple $\left(Y_{d q}, Y_{q q}, Y_{\text {batq }}\right)$ can be created by applying a perturbation in $V_{q} D C$ value. The procedure is identical to the creation of $V_{d}$ since the $V_{q}$ data are taken from the PLL figure. Although the validation of these 3 transfer functions is responsible for the change. The procedure is identical to the creation of $V_{d}$ since the $V_{q}$ data are taken from the PLL figure. Although the validation of these 3 transfer functions is responsible for the change in the phase angle and not on the peak amplitude of the system like in the $V_{d}$.

The identified functions created for the black-box modelling are listed in the table 3.3 in mathematical expressions. 

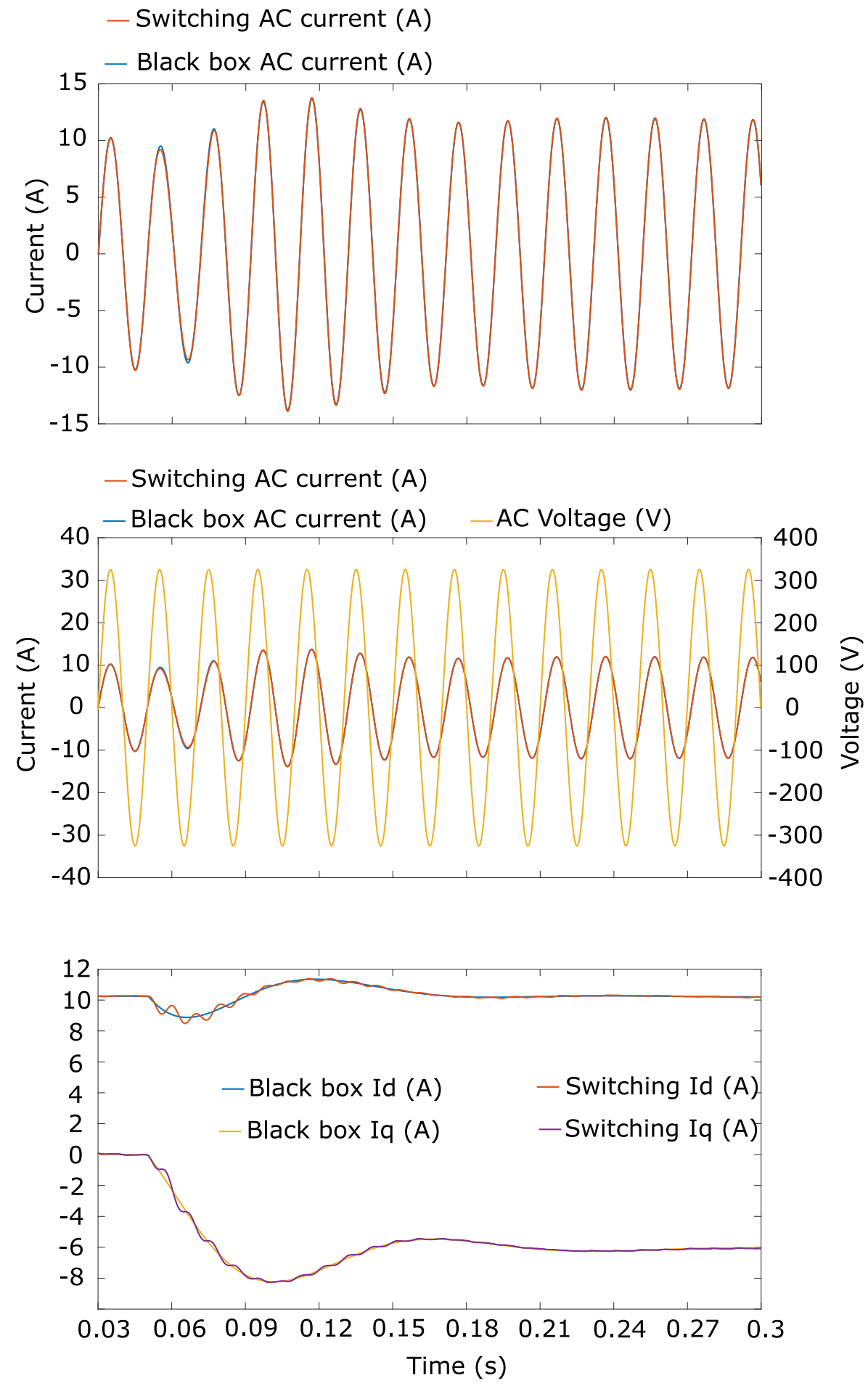

Figure 3.24 Results comparison between switching and black-box model for a step of $1 \mathrm{KVar}$ 

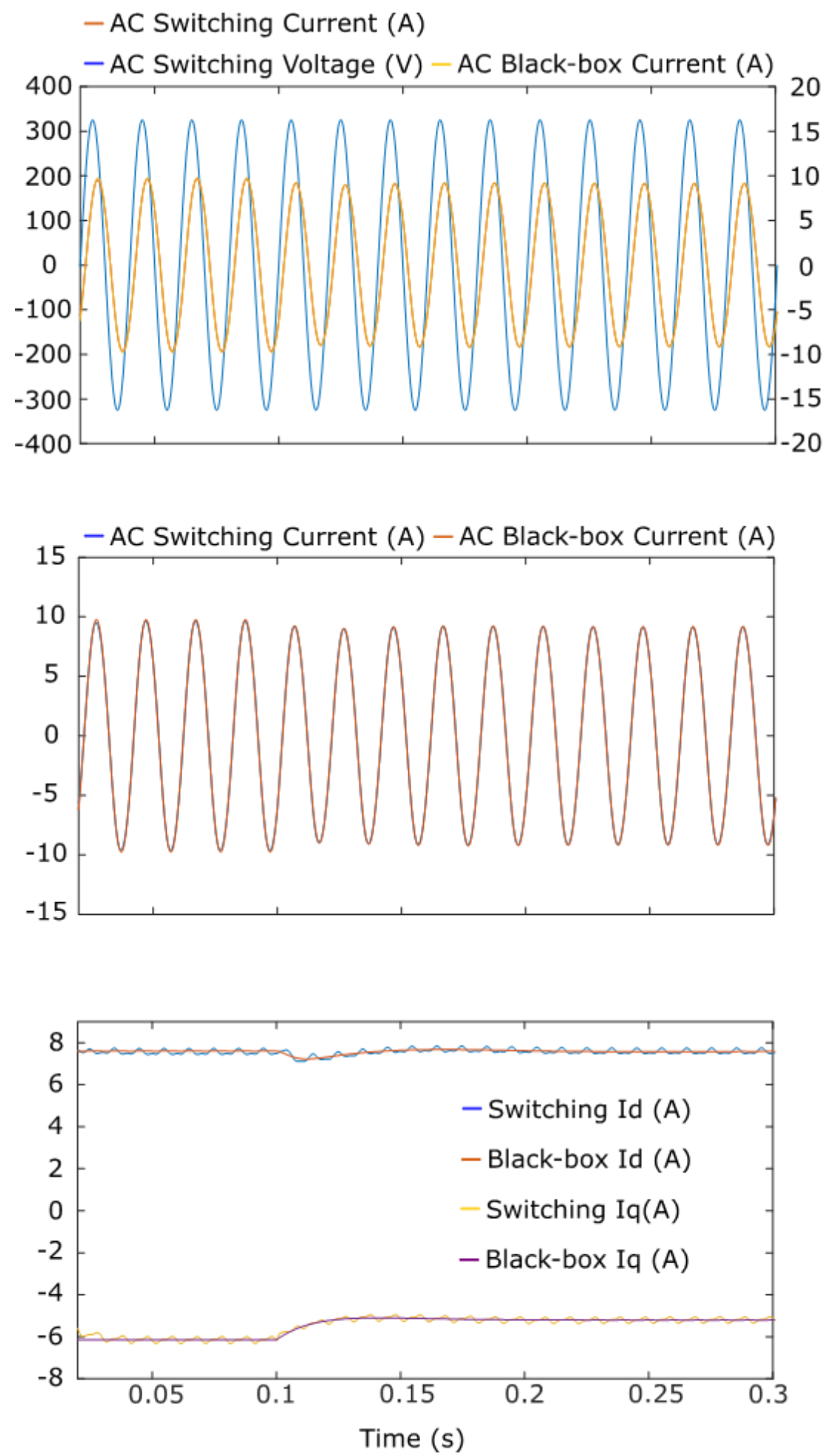

Figure 3.25 Results comparison between switching and black-box model for a step of 60V 

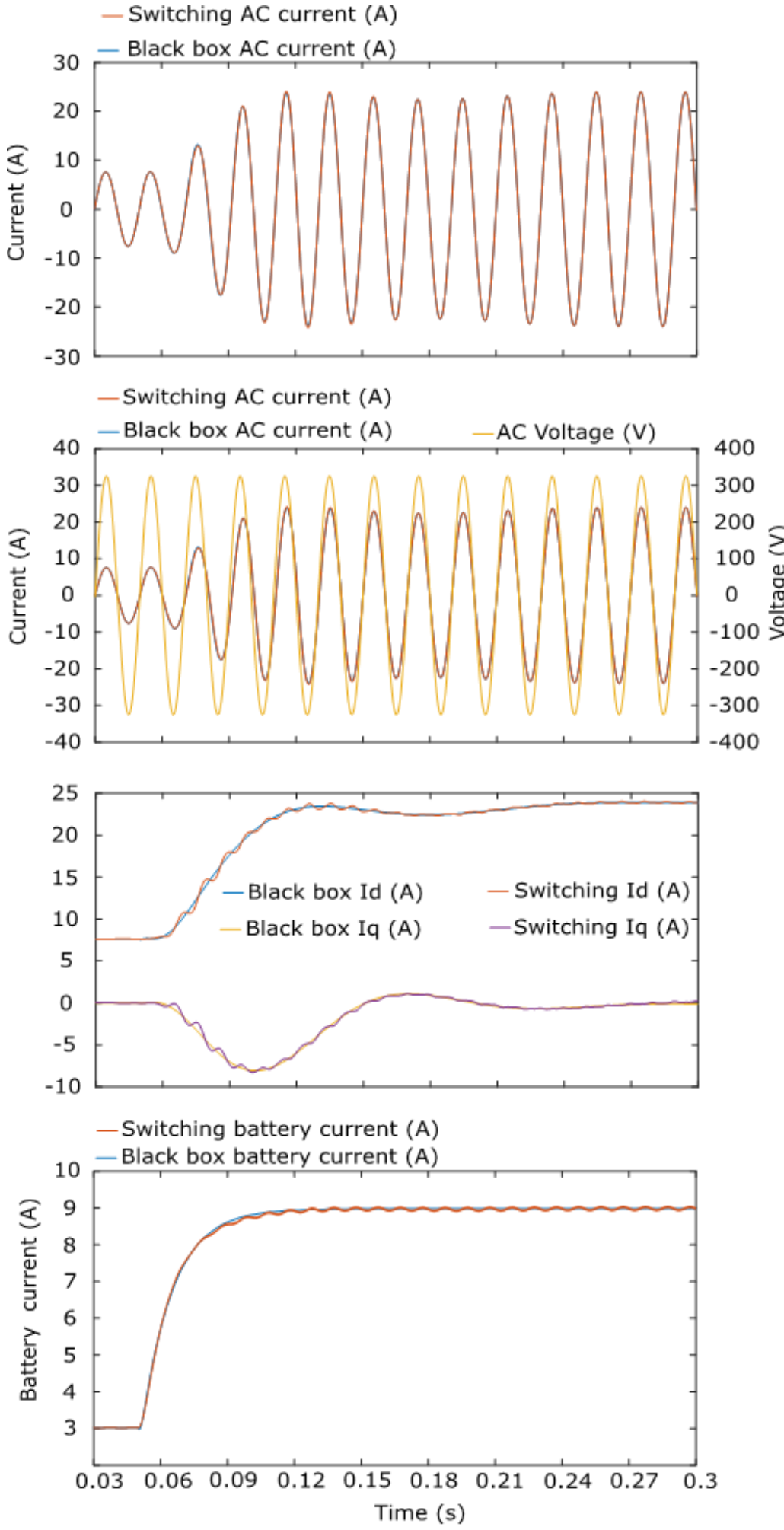

Figure 3.26 Results comparison between switching and black-box model for a step of $6 \mathrm{~A}$ 

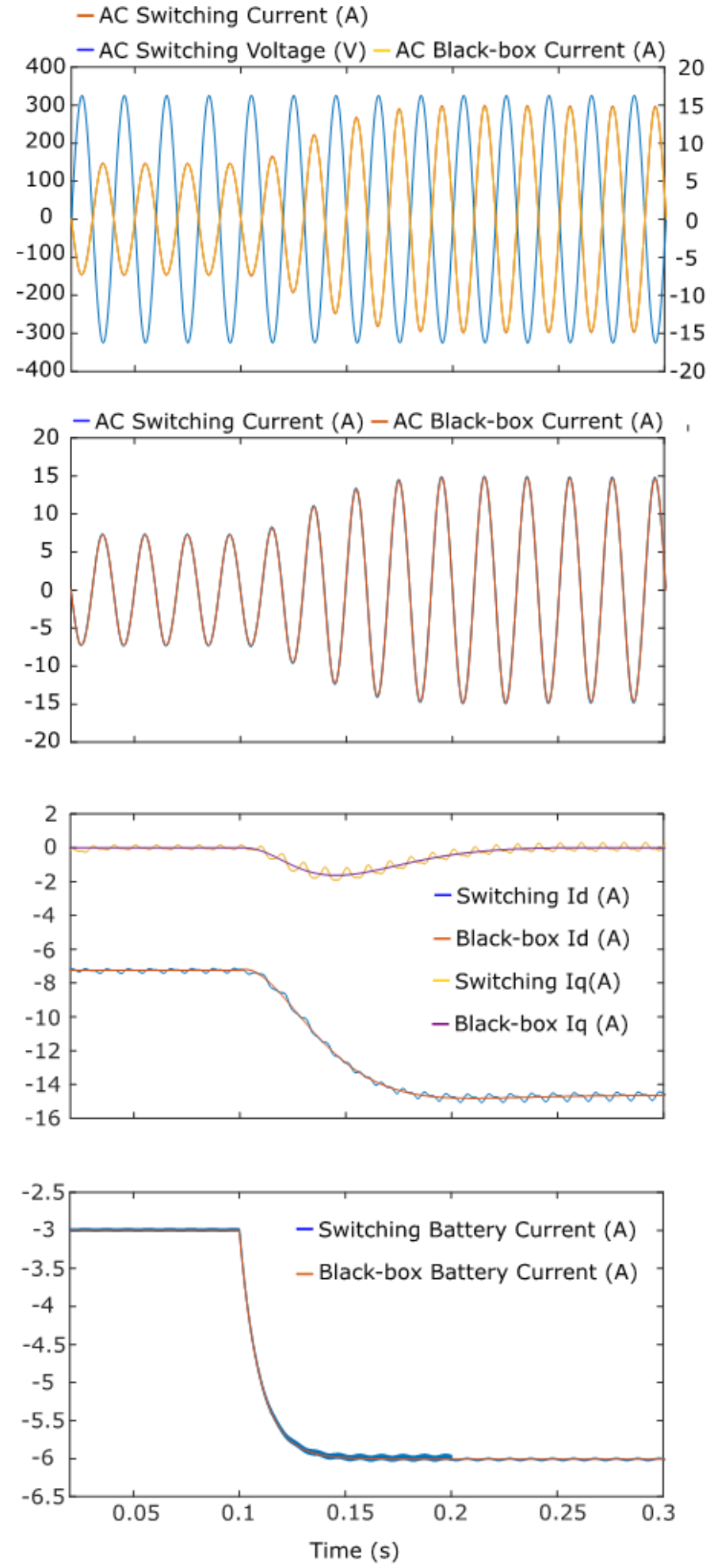

Figure 3.27 Results comparison between switching and black-box model for a step of -3A 

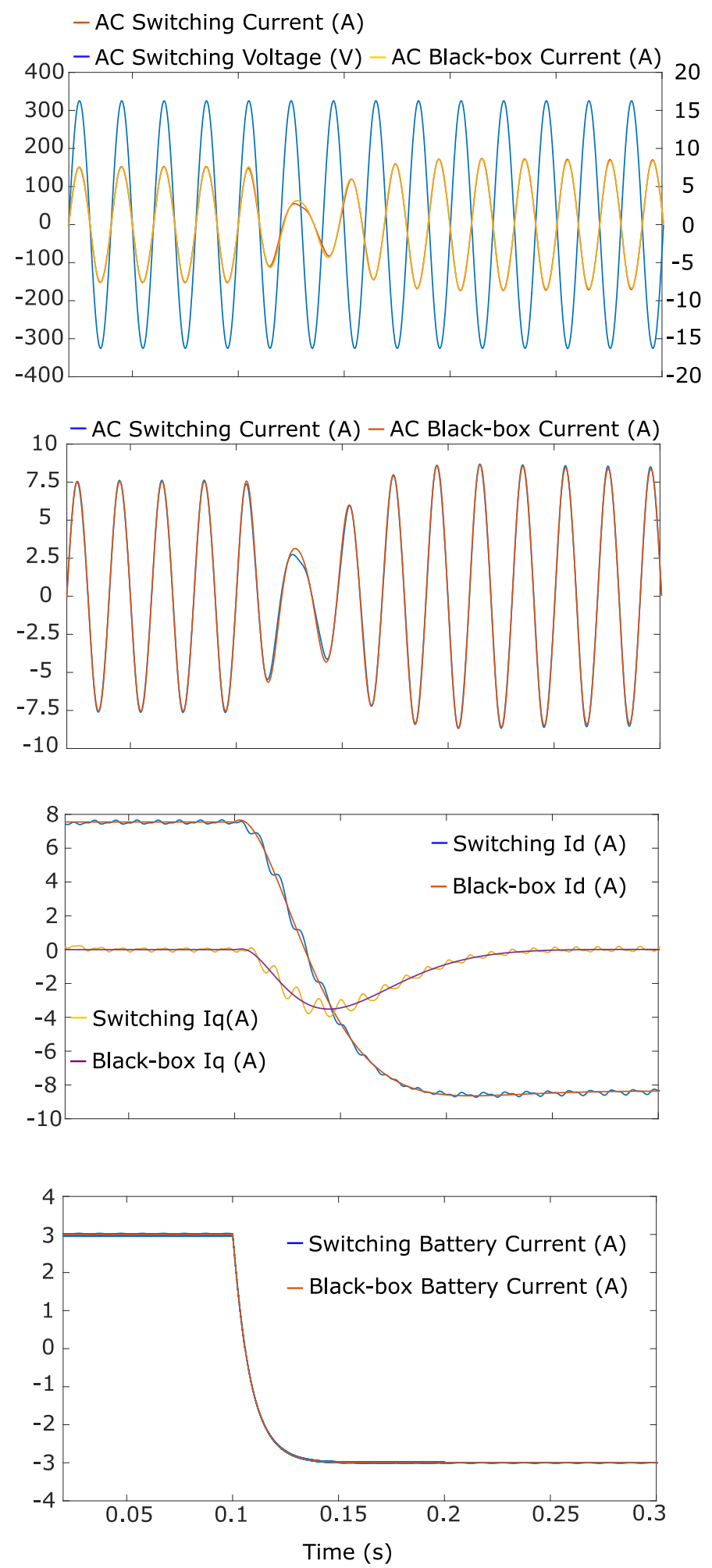

Figure 3.28 Results comparison between switching and black-box model for a step of -6A 


\begin{tabular}{|c|c|c|}
\hline Tests & Transfer Functions & Identified Transfer Functions \\
\hline \multirow{3}{*}{ Test 1} & $H_{d q}(\mathrm{~s})$ & $\frac{-0.1924 s^{2}+1.6411 s-3.519}{s^{3}+70.64 s^{2}+4340 s+69110}$ \\
\hline & $H_{q q}(s)$ & $\frac{-0.1924 s^{2}+1.6411 s-3.519}{s^{3}+70.64 s^{2}+4340 s+69110}$ \\
\hline & $H_{\text {batq }}(s)$ & Neglected \\
\hline \multirow{3}{*}{ Test 2} & $Y_{d q}(s)$ & $\frac{-588.7 s^{2}-1.041 e 4 s-3.927 e 4}{s^{4}+469.9 s^{3}+9.138 e 4 s^{2}+4.368 e 6+1.445 e 8}$ \\
\hline & $Y_{q q}(s)$ & $\frac{1.409 s+50.14}{s^{2}+100.5 s+3182}$ \\
\hline & $Y_{\text {batq }}(s)$ & Neglected \\
\hline \multirow{3}{*}{ Test 3} & $H_{\text {dref }}(s)$ & $\frac{-14 s^{2}+4051+1.285 e 5}{s^{3}+59.9 s^{2}+3605+4.7 e 4}$ \\
\hline & $H_{\text {qref }}(s)$ & $\frac{-0.155 s^{3}+48.39 s^{2}-6039 s-3845}{s^{3}+71.57+4218 s+9.977 e 4}$ \\
\hline & $H_{\text {batref }}(s)$ & $\frac{-30.46 s+6.21 e 4}{s^{2}+927.4 s+6.21 e 4}$ \\
\hline \multirow{3}{*}{ Test 4} & $H_{\text {dref }}(s)$ & $\frac{-14.77 s+5134}{s^{2}+70.15 s+2081}$ \\
\hline & $H_{\text {qref }}(s)$ & $\frac{6.884 e 5 s-6.651 e 4}{s^{4}+262.2 s^{3}+2.608 e 4 s^{2}+1.111 e 6+2.017 e 7}$ \\
\hline & $H_{\text {batref }}(s)$ & $\frac{107.1 s+1.653 e 4}{s^{2}+264.1 s+1.649 e 4}$ \\
\hline \multirow{3}{*}{ Test 5} & $H_{\text {dref }}(s)$ & $\frac{-16.09 s+5857}{s^{2}+74.34 s+2208}$ \\
\hline & $H_{\text {qref }}(s)$ & $\frac{-5568 s^{2}+2.408 e 6 s-1.99 e 5}{s^{4}+668.6 s^{3}+7.287 e 4 s^{2}+3.512 e 6+6.37 e 7}$ \\
\hline & $H_{\text {batref }}(s)$ & $\frac{80.16 s+1.971 e 5}{s^{2}+1747 s+1.971 e 5}$ \\
\hline
\end{tabular}

Table 3.3 Identified transfer functions from the switching model 


\subsection{Introduction of system level battery chargers}

In the previous sections, a step-by-step procedure was followed to create a fully operating bidirectional battery charger in power simulation (PSIM). The charger was composed of two converters, one boost rectifier and a dc-dc dual active bridge followed by a battery. The charger as it was also mentioned in the introduction it was neither the simplest nor the most complex. Also, a method for identification of this device has been implemented and is called black-box model. A method which can represent the procedure of a device accurately without any insight information only by collecting the input and output signals.

Following the literature review, one of the classifications on battery chargers is the on and off-boards which means chargers could exist outside and inside the vehicles. In this section two off-board chargers will take place in order to check the exchange of power between of more than one charger.

The battery charger indicated in the previous section will be used in the current part once more but in parts. One of the simplest bidirectional battery chargers is a boost rectifier, similar of the one which is fully explained in the section boost rectifier together with the controller. Two EV batteries as off-board chargers are connected through two individual dc-dc dual active bridges and with their mutual connection point to the boost rectifier. Once more the dc-dc dual active bridges and the EV batteries are identical with the previous section.

In the following scheme (figure 3.29) the idea of exchange power between the grid and two EV's indicated as batteries is shown. DC-DC converters are added as a middle point of dc bus between the batteries and the boost converter and then to the grid.

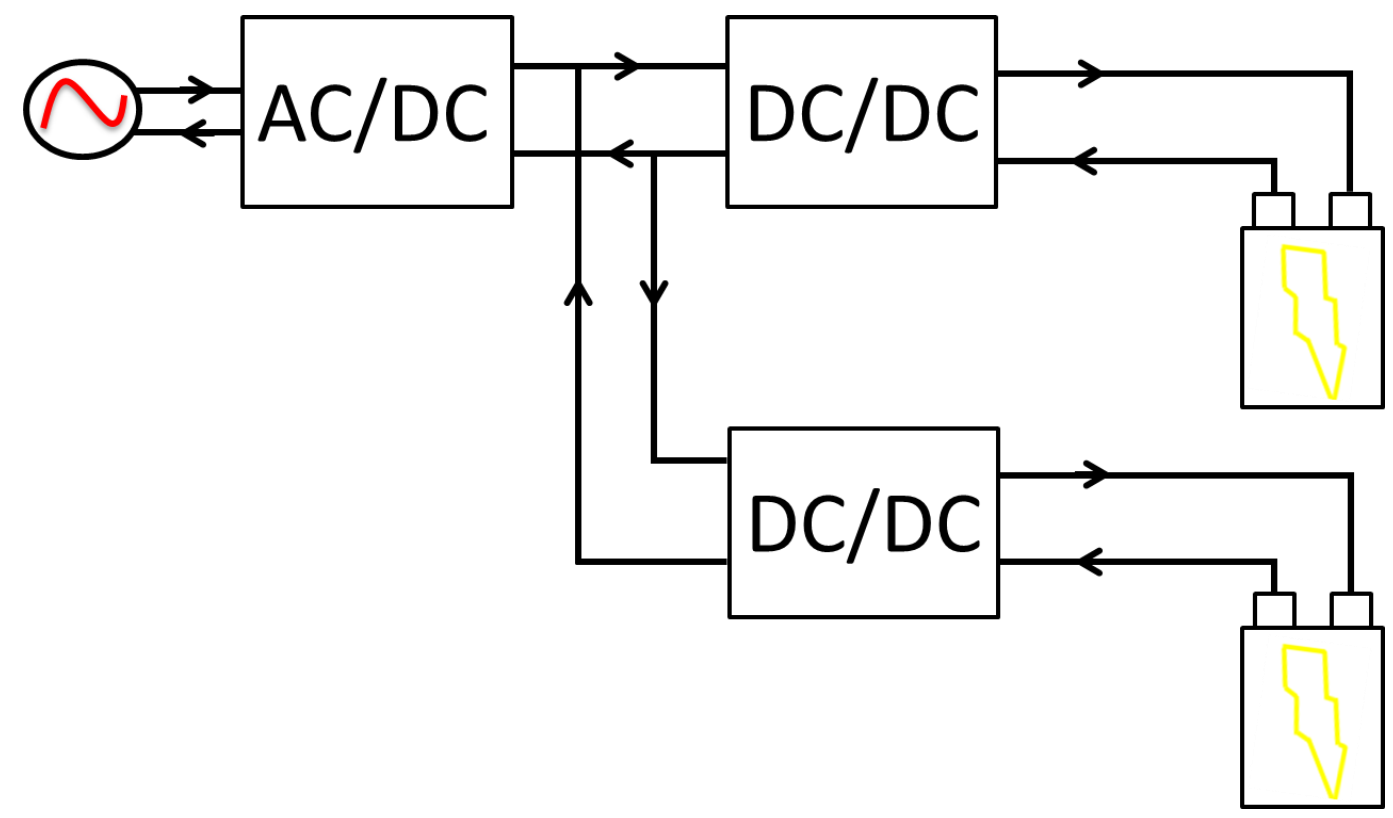

Figure 3.29 Block diagram of system level battery chargers 
Later a switching model in PSIM and an extensive procedure of exchange power will be portrayed accompanied with results from both sides of the circuit. As well as in the previous sections a black-box model of this system level will be implemented testing the accuracy between the model and the power simulation charger.

\subsection{Small-signal Black-box model for linear simulation of a system level battery chargers}

This scheme in figure 3.30 is an actual micrograph of a system level of battery charger. This number of vehicles would interact simultaneously with the grid and exchange power back and forward wherever there is a need. In this case which we examine the lowest level of system level will appear with two batteries representing two EV's without charger (off-board).

This circuit is similar to the bidirectional battery charger of the previous section. The only difference is the addition of an extra DC-DC converter along with an EV battery is added as an off-board charger. The two dc-dc converters are connected in a common point representing a dc bus and then to the boost rectifier followed by the grid.

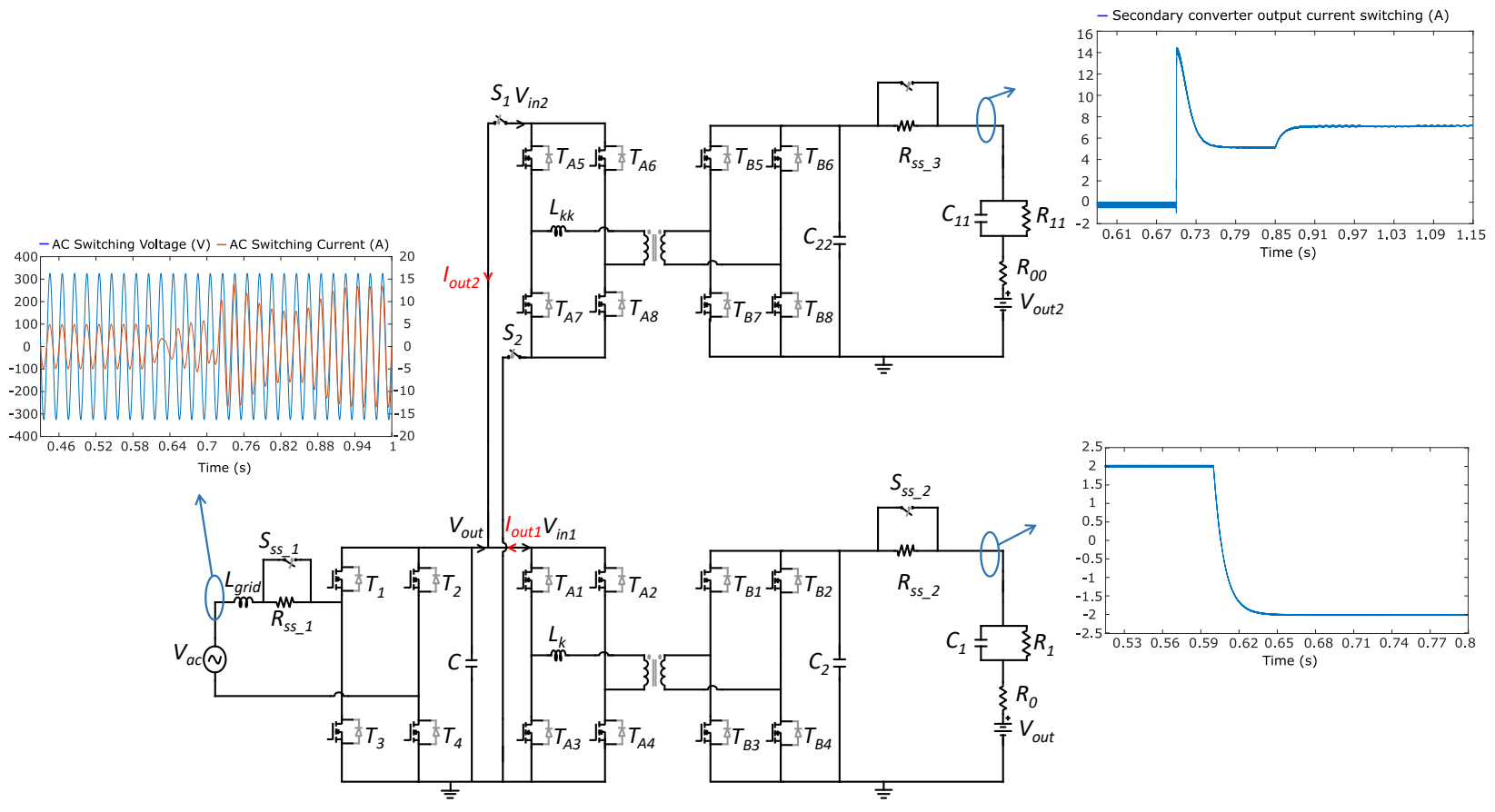

Figure 3.30 System level of 2 connected battery chargers 
The procedure of the small-scale system level starts from the point that is left in the previous section. The circuit scheme was the boost rectifier connected to the grid from the left side and on the right side the first dc-dc converter followed by the battery. The circuit is working at a certain power level. The secondary battery with its corresponding DAB converter is connected and working with different power level. In a designated time, the switches which are isolating the secondary battery from the rest of the circuit are closing and are entering the system.

In difference with the previous scheme, in the 'system level' scheme a clearer approach about identifying the small-signal black-box model will be followed. The scheme of figure 3.31 is decomposing the system level scheme into three parts, 2 DC/DC converters and 1 AC/DC. This decomposition is implemented for a better performance of the scheme since an addition of a secondary converter is added. As it derives the identification process will be followed for three black-boxes instead of one.

Since the $2 \mathrm{DC} / \mathrm{DC}$ converters are identical the tests will be implemented only once. In the left side of the $D C / D C$ converters a DC source of $400 \mathrm{~V}$ is added in order to simulate the constant voltage which exists in the connection between them. The input variables of the $2 \mathrm{DC} / \mathrm{DC}$ converters are the current control references ( $I_{\text {ref1 }}$ for the primary converter and $I_{\text {ref2 }}$ for the secondary) and the input voltages ( $V_{\text {in } 1}$ for primary and $V_{\text {in2 }}$ for secondary). The input variables $\left(I_{\text {ref1 }}, I_{\text {ref2 }}, V_{\text {in } 1}, V_{\text {in2 }}\right.$ ) are subtracted by the respective nominal values $\left(I_{\text {ref1 }}{ }^{d c}, I_{\text {ref2 }}{ }^{d c}, V_{\text {in } 1}{ }^{d c}, V_{\text {in2 }}{ }^{d c}\right)$ giving a small signal perturbation of the input variables in the black-box model. In the output the respective nominal values $\left(I_{\text {bat1 }}{ }^{d c}, I_{\text {out1 } 1}{ }^{d c}, I_{\text {bat2 }}{ }^{d c}, I_{\text {out2 }}{ }^{d c}\right)$ are added to the output perturbations giving the output variables. The output variables consist of the output battery currents ( $I_{\text {bat1 }}$ for the primary and $I_{\text {bat2 }}$ for the secondary) on the batteries side and the output currents ( lout1 $_{1}$ primary, $\mathrm{I}_{\text {out2 }}$ secondary) as the currents connected to the DC part of the AC/DC converter.

The AC/DC converter has 4 inputs, 2 on the $A C$ side consisted of $\mathrm{I}_{\mathrm{ac}}$ and $\mathrm{V}_{\mathrm{ac}}$ as current and voltage of the grid and on the DC side $V_{\text {outref }}$ controlling the bus voltage at $400 \mathrm{~V}$ and $\mathrm{Q}_{\text {ref }}$ as the reference of reactive

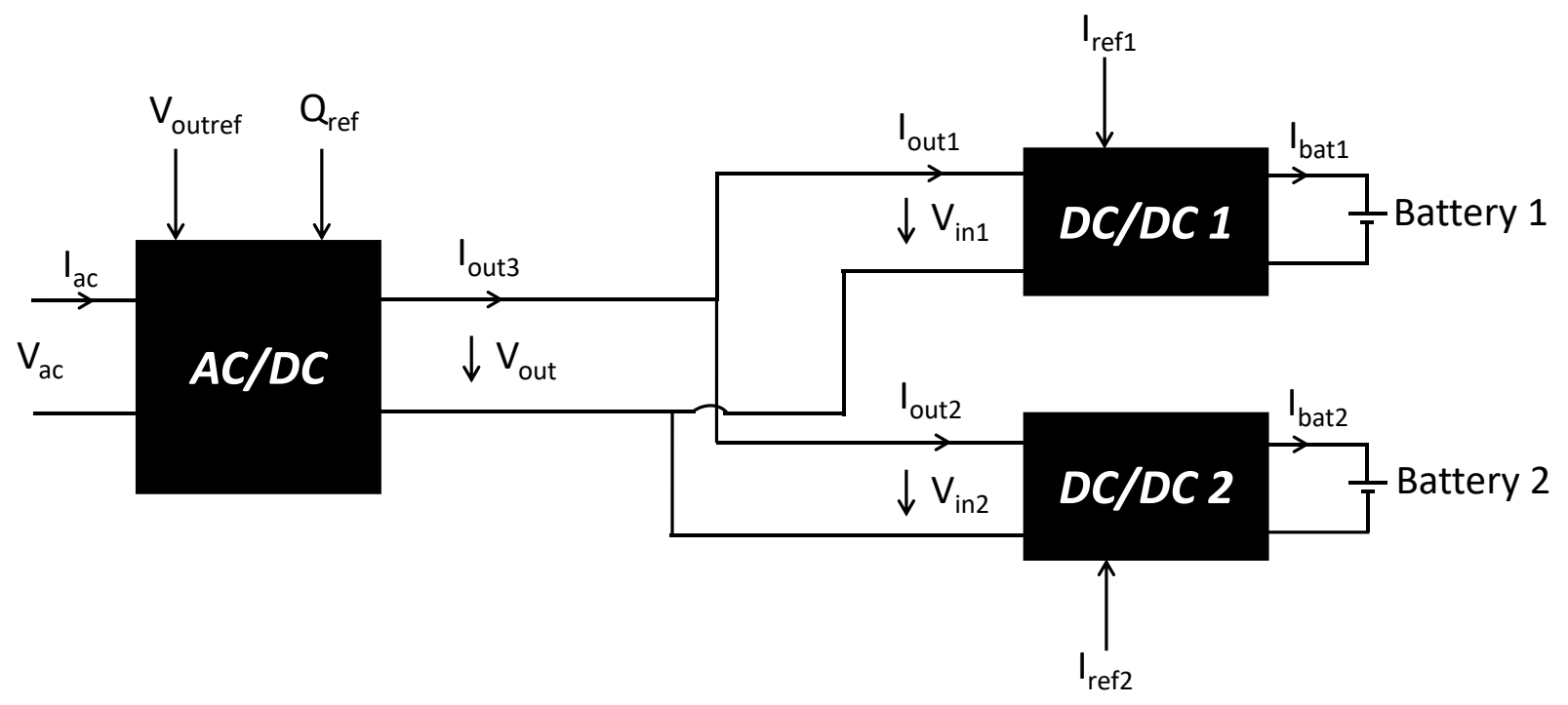

Figure 3.31 Block Diagram of the battery charger with two batteries 


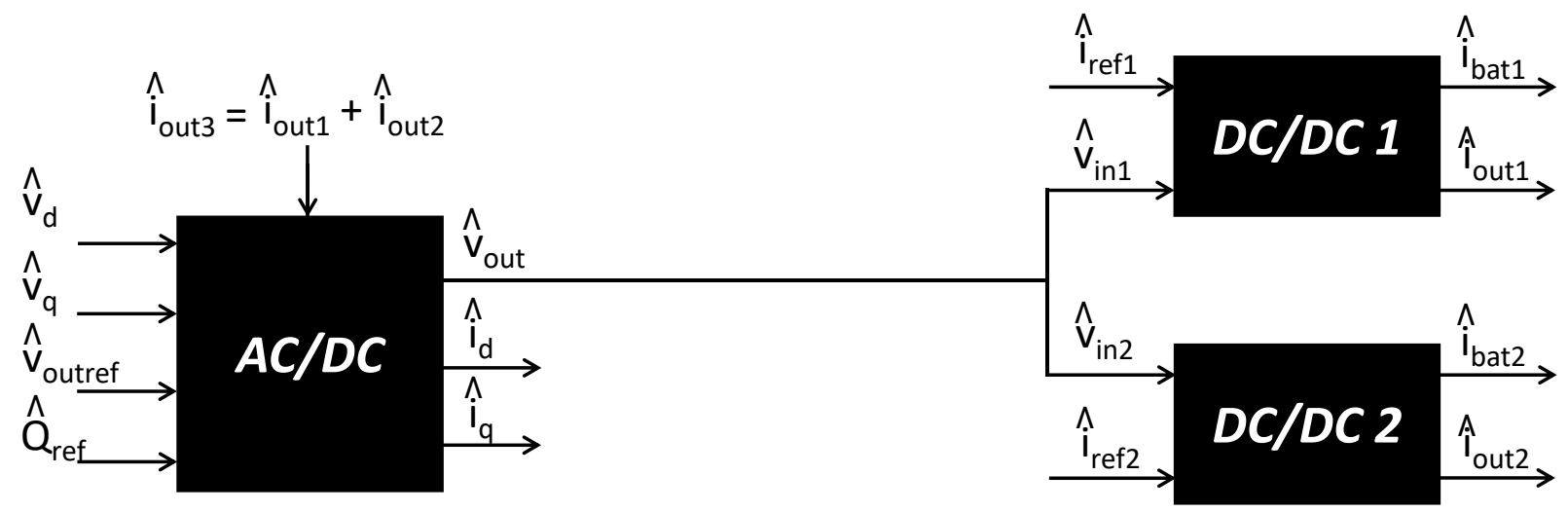

Figure 3.32 Block diagram of the black-box model showing the perturbation of the input and output variables

power. The summation of the $I_{\text {out1 }}$ and $I_{\text {out } 2}$ introduces $I_{\text {out3 }}$ as an input in the DC part of the AC/DC converter. $\mathrm{I}_{\text {out3 }}$ is entering the converter and then the current it seems to split in 2 parts (Figure 3.31).

In the figure 3.32, d-q framework perturbation currents $\left(\hat{\imath}_{\mathrm{d}}, \hat{l}_{\mathrm{q}}\right)$ and voltages $\left(\hat{v}_{\mathrm{d}}, \hat{v}_{\mathrm{q}}\right)$ are shown in the output and input respectively of the boost converter block diagram. These values come from rotation block of $\alpha \beta / d q$ and nominal values $I_{d}{ }^{d c}, I_{q}{ }^{d c}, V_{d}{ }^{d c}, V_{q}{ }^{d c}$ are subtracted giving out the $d$-q perturbation values. The output voltage perturbation ( $\left.\hat{v}_{\text {out }}\right)$ is obtained from the subtraction of the nominal output voltage $\left(V_{\text {out }}{ }^{d c}\right)$, the voltage which returns back to the 2 input voltages of the $D C / D C$ converters $\left(V_{\text {in1 }}\right.$ and $\left.V_{\text {in2 } 2}\right)$.

In the equations (E 3.42a, E 3.42b) the transfer functions procedure of the $2 \mathrm{DC} / \mathrm{DC}$ converters is shown. The operation point of the reference control currents is set at $-2 \mathrm{~A}$ and a small perturbation of $-0.5 \mathrm{~A}$ is applied. The same procedure is applied in both input voltage with a steady state operation point set in $400 \mathrm{~V}$ is affected by a small perturbation of $80 \mathrm{~V}$ while the control reference current is in steady state conditions. The small signal perturbations added one per time in each input signal affect 4 different outputs. Therefor in the end 8 transfer functions are collected presenting the dynamic response of the 2 DC/DC converters. The subindex in the right hand side it shows the inputs of the 2 DC/DC converters $\left(\hat{v}_{\text {in } 1}\right.$ , $\left.\hat{v}_{\text {in2 }}, \hat{l}_{\text {ref1 }}, \hat{l}_{\text {ref2 }}\right)$ and on the left their output $\left(\hat{l}_{\text {bat1 }}, \hat{l}_{\text {bat2 }}, \hat{l}_{\text {out1 }}, \hat{l}_{\text {out2 } 2}\right)$ while in the middle their linear transfer functions ( $Y_{\text {bat1in1 }}, H_{\text {bat1ref1, }} Y_{\text {bat2in2 }}, H_{\text {bat2ref2, }}, Y_{\text {out1in1 }}, H_{\text {out1ref1 }}, Y_{\text {out2in2 }}, H_{\text {out2ref2 }}$ ).

$$
\begin{aligned}
& \left(\begin{array}{l}
\hat{\imath}_{\text {bat } 1}(s) \\
\hat{\imath}_{\text {out } 1}(s)
\end{array}\right)=\left(\begin{array}{l}
Y_{\text {bat } 1 \text { in } 1}(s) H_{\text {bat } 1 \text { ref } 1}(s) \\
Y_{\text {out } 1 \text { in } 1}(s) H_{\text {out } 1 \text { ref } 1}(s)
\end{array}\right) \cdot\left(\begin{array}{c}
\hat{v}_{\text {in } 1}(s) \\
\hat{\imath}_{\text {ref } 1}(s)
\end{array}\right) \\
& \left(\begin{array}{l}
\hat{l}_{\text {bat } 2}(s) \\
\hat{\imath}_{\text {out } 2}(s)
\end{array}\right)=\left(\begin{array}{l}
Y_{\text {bat } 2 \text { in } 2}(s) H_{\text {bat } 2 r e f 2}(s) \\
Y_{\text {out } 2 \text { in } 2}(s) H_{\text {out } 2 r e f 2}(s)
\end{array}\right) \cdot\left(\begin{array}{c}
\hat{v}_{\text {in } 2}(s) \\
\hat{\imath}_{\text {ref } 2}(s)
\end{array}\right)
\end{aligned}
$$


In the same pattern the AC/DC converter is tested as in DC/DC converters. Here there is only one input and consequently there is no need of bear in mind the equation (E 3.41). The rest of the AC/DC converter inputs $\left(\hat{v}_{\mathrm{d}}, \hat{v}_{\mathrm{q}}, \hat{v}_{\text {outref }}, \hat{Q}_{\text {ref }}\right)$ are assumed to kept constant. In the AC/DC converter the response from the test is added -after removing the starting nominal current and place it initially to 0 - on the current DC output of the converter. In equation (E 3.43) the input of AC/DC converter is indicated as $\hat{l}_{\text {out3, }}$, the output signals as $\hat{v}_{\text {out }}, \hat{l}_{\mathrm{d}}, \hat{\imath}_{\mathrm{q}}$ and the dynamic responses as $\mathrm{Y}_{\text {outout3 }}, \mathrm{H}_{\text {dout3 }}, \mathrm{H}_{\text {qout3 }}$ respectively. The perturbation in order to obtain transfer function was $0.5 \mathrm{~A}$.

$$
\left(\begin{array}{c}
\hat{v}_{\text {out }}(s) \\
\hat{i}_{d}(s) \\
\hat{i}_{q}(s)
\end{array}\right)=\left(\begin{array}{c}
Y_{\text {outout } 3}(s) \\
H_{\text {dout } 3}(s) \\
H_{\text {qout } 3}(s)
\end{array}\right) \cdot\left(\hat{i}_{\text {out } 3}(s)\right)
$$

All transfer functions have been identified with transfer function models in a continuous time and are indicated in the table 3.4. After the identification of the 3 converters the $2 \mathrm{DC} / \mathrm{DC}$ converters are added with the aid of 2 current source controllers while the $A C / D C$ with a voltage source controller. The addition of the 2 currents from the DC/DC converters ( $\hat{l}_{\text {out1 }}$ form DC/DC1 and $\hat{l}_{\text {out2 }}$ from DC/DC2) is indicated as lout3 and being introduced as an input in the $D C$ part of the $A C / D C$ converter. The return of the voltage ( $\left.\hat{v}_{\text {out }}\right)$ is done through the voltage controller back to the voltage inputs $\left(V_{i n 1}\right.$ and $\left.V_{i n 2}\right)$ of the DC/DC converters.

The contribution of the system level subchapter is the development of the black-box strategy not only for a single stage converter as before but reaching a higher degree of complexity connecting a number of secondary converters to the primary converter and prove the accuracy of the system with black-box strategy. This concept is achieved by adding 2 dc-dc converters in parallel connected to a dc bus with the primary ac-dc converter. Following the comparison of the PSIM simulation model representing a switching model will be compared with the black-box model created in Matlab which will show the accuracy of this strategy. 


\begin{tabular}{|c|c|c|}
\hline Output/Input & $\begin{array}{l}\text { Transfer } \\
\text { functions }\end{array}$ & Identified Transfer Functions \\
\hline $\mathrm{I}_{\text {out } 1} / \mathrm{I}_{\mathrm{ref1}}$ & $\mathrm{H}_{\text {out } 1 \text { ref1 } 1(\mathrm{~s})}$ & $\frac{8.113 s+1.122 e 5}{s^{2}+1072 s+1.118 e 5}$ \\
\hline $\mathrm{I}_{\text {out } 1} / \mathrm{V}_{\text {in } 1}$ & $\mathrm{Y}_{\text {outlin1 } 1}(\mathrm{~s})$ & Neglected \\
\hline $\mathrm{I}_{\text {bat } 1} / \mathrm{I}_{\mathrm{ref1}}$ & $\mathrm{H}_{\text {bat Iref1 }}(\mathrm{s})$ & $\frac{-6.196 s+1.552 e 6}{s^{2}+1.362 e 4 s+1.552 e 6}$ \\
\hline $\mathrm{I}_{\text {bat } 1} / \mathrm{V}_{\text {in1 }}$ & $\mathrm{Y}_{\text {batlin1 }}(\mathrm{s})$ & Neglected \\
\hline $\mathrm{I}_{\text {out } 2} / \mathrm{I}_{\mathrm{ref} 2}$ & $\mathrm{H}_{\text {out2ref2 }}(\mathrm{s})$ & $\frac{-16.55 s+1.683 e 5}{s^{2}+1537 s+1.591 e 5}$ \\
\hline $\mathrm{I}_{\text {out } 2} / \mathrm{V}_{\text {in } 2}$ & $\mathrm{Y}_{\text {out2in2 }}(\mathrm{s})$ & $\frac{-1.746 e-3 s^{5}+286.9 s^{4}+5.387 e 4 s^{3}+1.317 e 6 s^{2}+5.277 e 7 s+9.148 e 8}{s^{5}+8497 s^{4}+1.472 e 6 s^{3}+8.501 e 7 s^{2}+1.518 e 9 s+7.184 e 10}$ \\
\hline $\mathrm{I}_{\mathrm{bat} /} / \mathrm{I}_{\mathrm{ref} 2}$ & $\mathrm{H}_{\text {bat2ref2 }}(\mathrm{s})$ & $\frac{-6.196 s+1.552 e 6}{s^{2}+1.362 e 4 s+1.552 e 6}$ \\
\hline $\mathrm{I}_{\text {bat2 }} / \mathrm{V}_{\text {in2 }}$ & $Y_{\text {bat2in2 }}(\mathrm{s})$ & $\frac{-1.746 e-3 s^{5}+286.9 s^{4}+5.387 e 4 s^{3}+1.317 e 6 s^{2}+5.277 e 7 s+9.148 e 8}{s^{5}+8497 s^{4}+1.472 e 6 s^{3}+8.501 e 7 s^{2}+1.518 e 9 s+7.184 e 10}$ \\
\hline $\mathrm{V}_{\text {out }} / \mathrm{I}_{\text {out3 }}$ & $\mathrm{Y}_{\text {outout3 }}(\mathrm{s})$ & $\frac{-100.8 s^{3}-4397 s^{2}-1.138 e 5 s-6.91 e 5}{s^{4}+75.78 s^{3}+3392 s^{2}+5.45 e 4 s+7.758 e 5}$ \\
\hline $\mathrm{I}_{\mathrm{d}} / \mathrm{I}_{\text {out3 }}$ & $\mathrm{H}_{\text {dout3 } 3}(\mathrm{~s})$ & $\frac{4.35 e 4 s+1.077 e 7}{s^{3}+1641 s^{2}+1.274 e 5 s+4.113 e 6}$ \\
\hline $\mathrm{I}_{\mathrm{q}} / \mathrm{I}_{\text {out3 }}$ & $\mathrm{H}_{\text {qout3 }}(\mathrm{s})$ & $\frac{7188 s-5841}{s^{3}+160.4 s^{2}+9131 s+1.798 e 5}$ \\
\hline
\end{tabular}

Table 3.4 Identified transfer functions from the system level model

\subsubsection{Comparison of small-signal black-box and switching model}

The last part of identification process of creating a black-box model is the validation part. In this part a test will be implemented with an extensive bandwidth of operations showing the accurate operation of this system level battery charger connection. In more detail the battery currents of the primary and secondary batteries will be shown along with the AC current responses.

The changes on the circuit have been set exclusively from the reference current point of the two dc-dc converters controllers. A single test implemented in the current section but with an extensive bandwidth of operations as it is shown below. The transfer functions have been estimated in a time domain with sample time 1e-6 following the simulation (PSIM) model.

The test starts with the primary DC/DC converter and the AC/DC converter operating in grid to vehicle mode with an operating point set at $2 \mathrm{~A}$ controlled by the primary reference current control $\mathrm{I}_{\text {ref1 }}$. In $0.6 \mathrm{~s}$ a step of $-4 A$ applies in the $I_{\text {ref1 }}$ causing a change of operating mode from grid to vehicle to vehicle to grid and resulting with a current of $-2 \mathrm{~A}$ in the primary battery current. In figure 3.33 this operation is shown with black-box model to be indicated in orange colour and PSIM switching model in blue. 


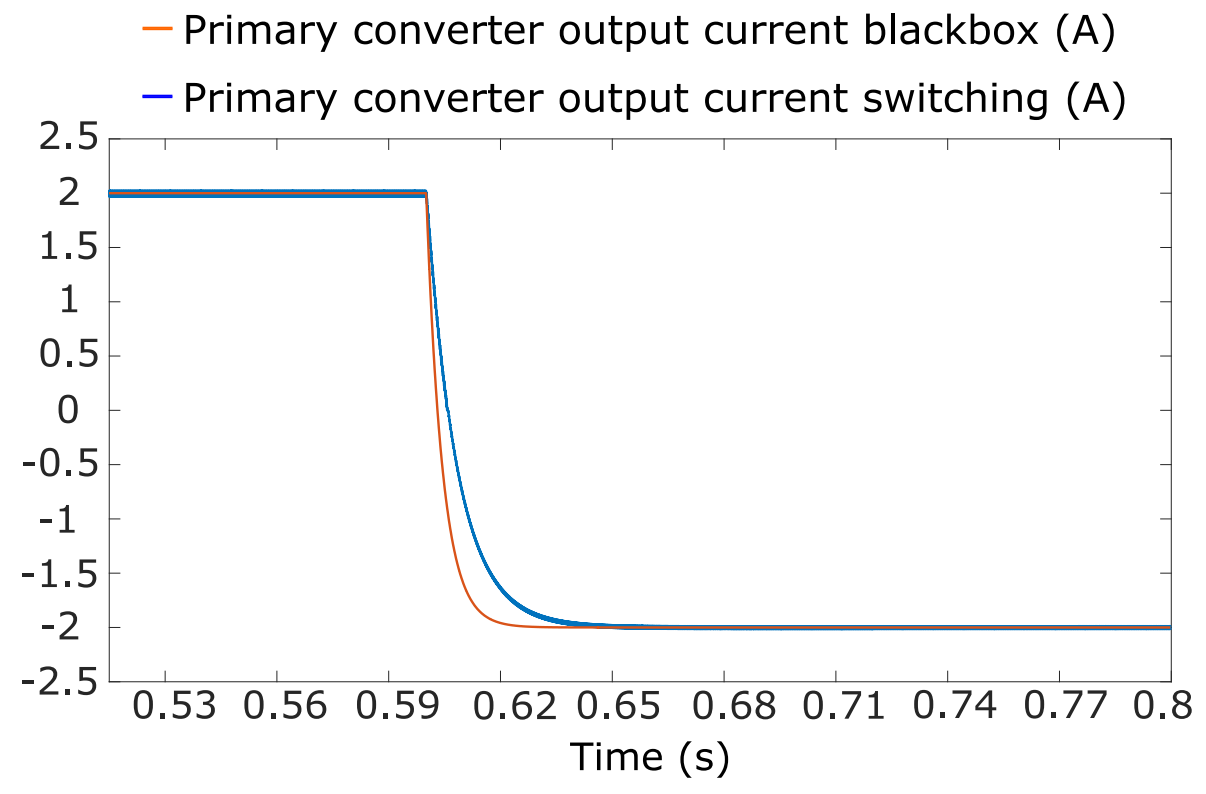

Figure 3.33 Results comparison between switching and black-box model for DC output of primary DC-DC converter

- Secondary converter output current blackbox (A)

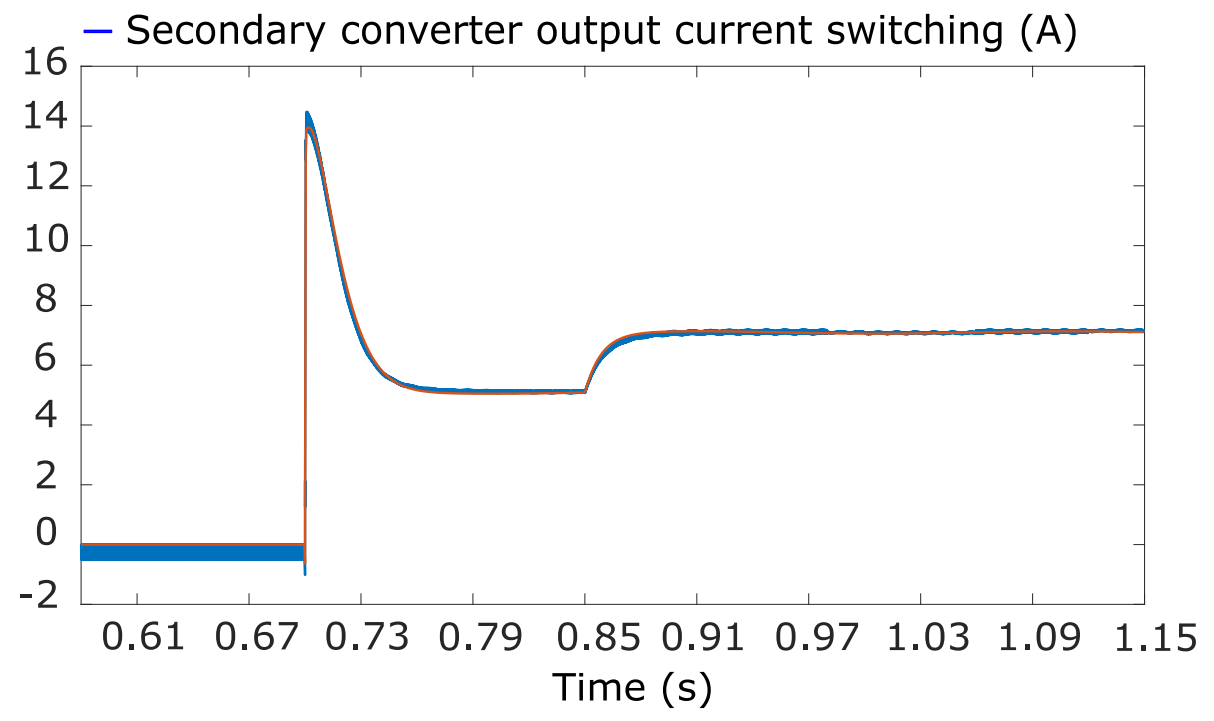

Figure 3.34 Results comparison between switching and black-box model for DC output of secondary DC-DC converter

The next step acts at $0.7 \mathrm{~s}$ adding the second DC/DC converter with the EV battery by activating the switches and entering the primary scheme. This second DC/DC converter it was already set at $5 \mathrm{~A}$ in grid to vehicle mode with the secondary reference current control $I_{\text {ref2 }}$. 

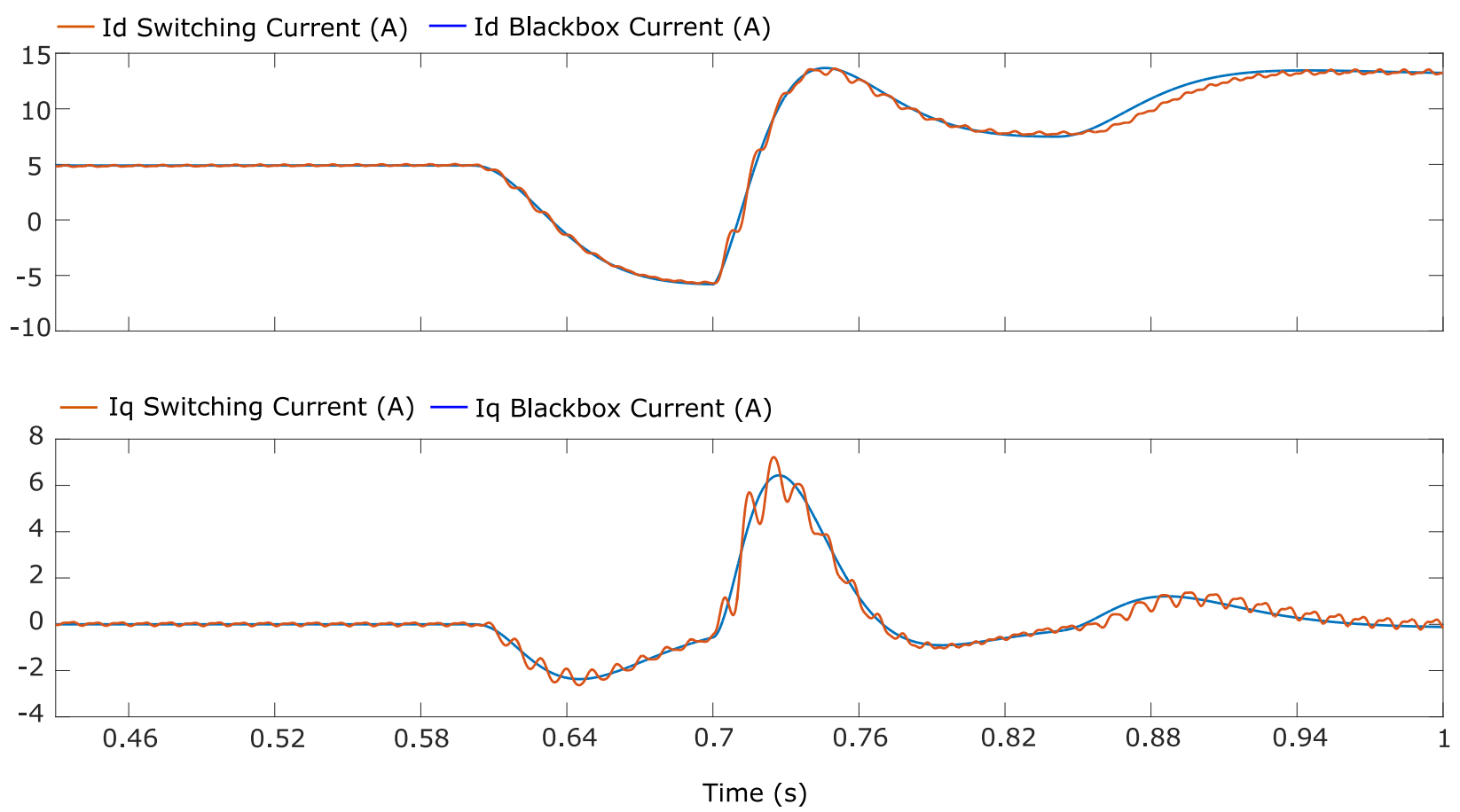

Figure 3.35 Results comparison between switching and black-box model for AC output of Current
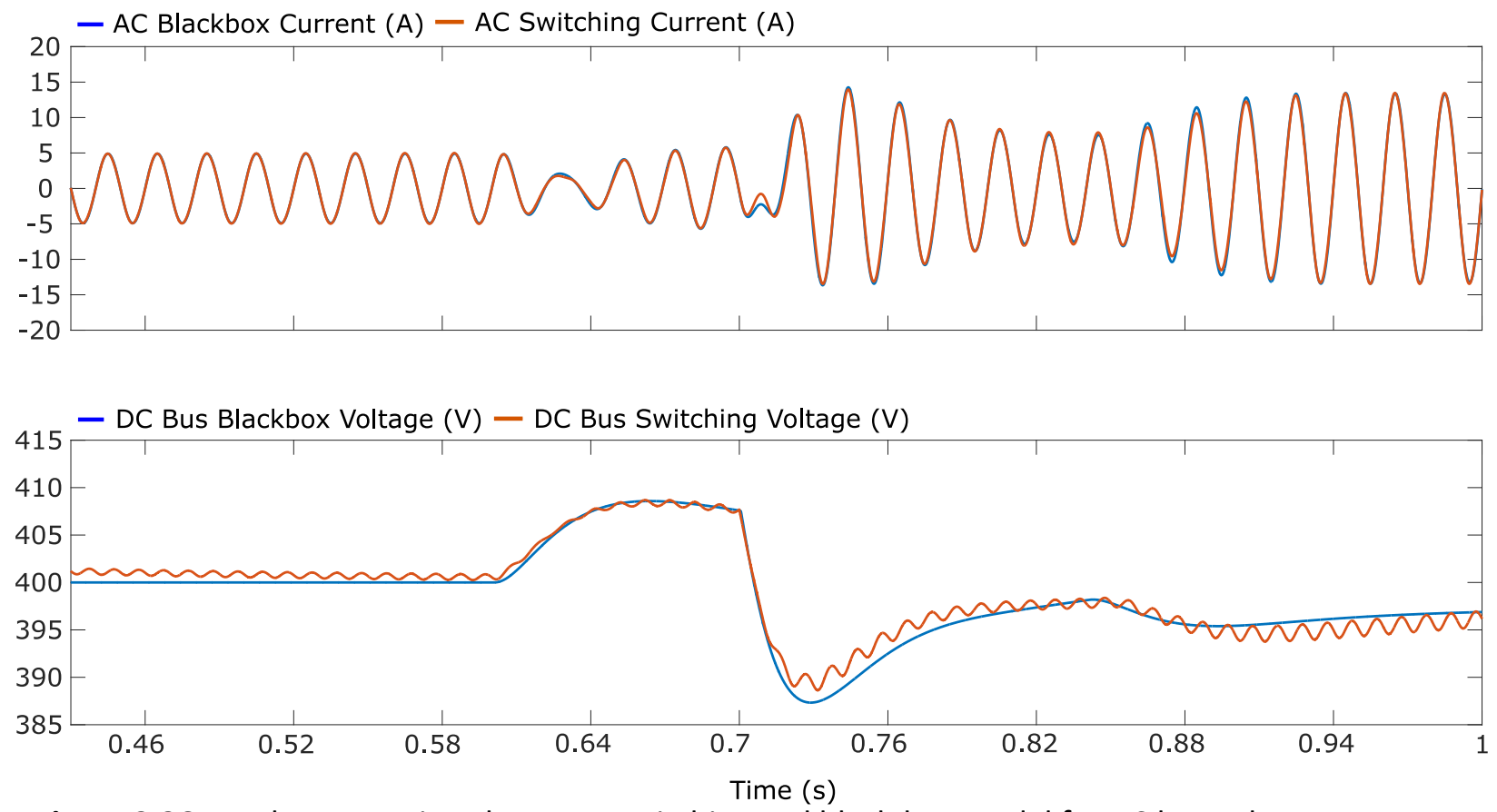

Figure 3.36 Results comparison between switching and black-box model for DC bus voltage connection point 
The summation of the 2 different currents changes once more the mode of operation to grid to vehicle. The final step is a $2 \mathrm{~A}$ step at $0.85 \mathrm{~s}$ in the secondary reference current control $\mathrm{I}_{\text {ref2 }}$ concluding the summing current at 7A in a grid to vehicle mode. Figure 3.34 the operation in the secondary battery current is shown through the experiment. As it can be seen before $0.7 \mathrm{~s}$ no movement of current is appeared. The reaction of the summation it is shown with a pick of current at $0.7 \mathrm{~s}$ launches the operation of this part. In orange colour it is shown once more the black-box model and in blue the PSIM switching model.

Figure 3.35 is shown the resulting $A C$ current response during the test. In the upper figure it can be seen the AC grid current (in orange) from PSIM switching model and the black-box model (in blue) of the AC current showing the accuracy of the model. In the bottom of the graph of the figure, the voltage in the connection point of the two dc-dc converters is illustrated in orange colour along with the black-box model in blue. Moreover, the $d-q$ components of the AC switching current are displayed in orange with their respective model in blue in figure 3.36 .

All the results show a very good accuracy of the black-box model compared with switching model used as a reference. These results will be validated again experimentally in the next chapter. 


\section{CHAPTER}

\section{Experimental results}

\subsection{Introduction}

The main purpose of this chapter is the evaluation of the proposed Behavioural Small-signal and Largesignal Black-box models for unidirectional and bidirectional battery chargers through their comparison with experimental results. To some extend in chapter 3 , the accuracy of the proposed models has been already tested through virtual prototyping using the PSIM simulator as the valid reference to be compared with the outcomes generated by the black-box models. In practice, in chapter 3 , it is a comparison among behavioural models and switching models, assuming that switching models are accurate enough to be established as the reference.

But now, it is time to avoid any proxy and represent the actual converters by themselves in an experimental setup, making the desired comparison fair. On the other hand, it is true that very often the experimental setup includes parasitic that are not estimated properly by the models, but in our approach, the model is generated through different tests in the actual converter, and this implies, that in principle, most of the characteristics of the converter, including the parasitic, would be identified by the model. In this statement, the bandwidth limitation due to the finite rising time of the applied step functions is not considered.

In this section, 2 sets of experiments will be described. In both, the bidirectional battery charger is represented by a commercial CINERGIA B2C30 (Bidirectional DC source 27kW/30A per channel/90A). This is a very flexible power source able to work in many operating modes. In the two experimental setups the power source operates as bidirectional AC/DC power converter behaving as a current source in the DC side. This means that the output DC current is controlled independently of the load. When the DC current reference is set to a positive value, the converter is operating in the G2V (Grid to Vehicle) mode, charging the battery. On the other hand, when the DC current reference is set to a negative value, the power converter is operating in V2G (Vehicle to Grid) mode giving energy from the DC side to the grid. Power source CINERGIA B2C30 is represented in figure 4.1. 


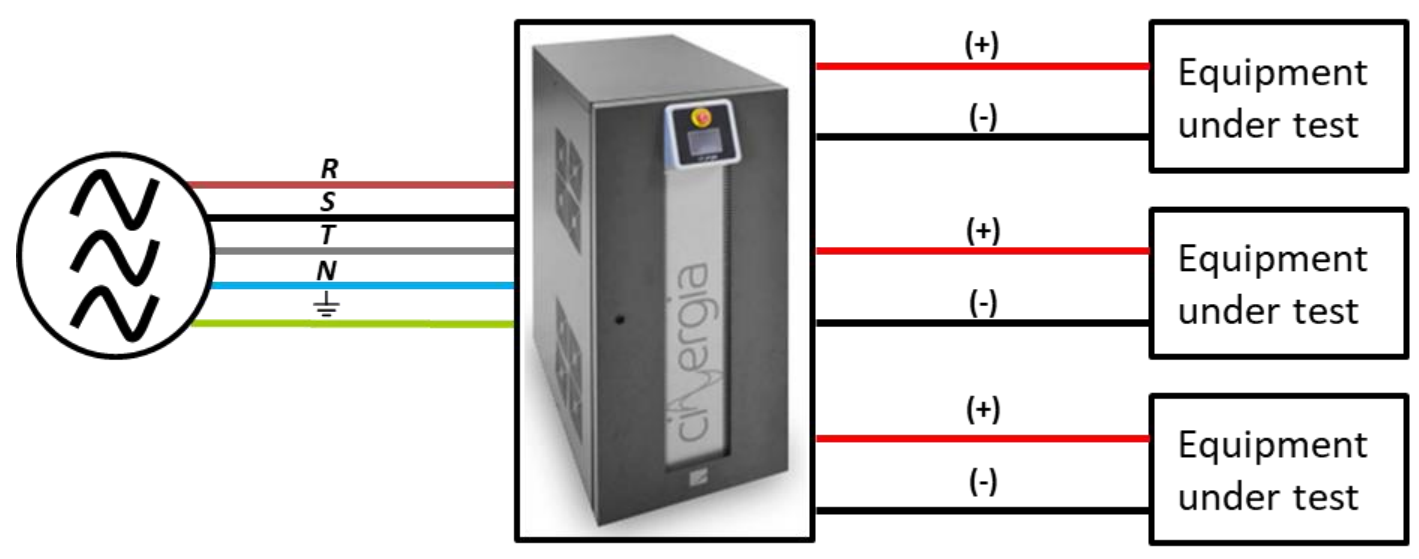

Figure 4.1 CINERGIA B2C30 independent unipolar mode dc outputs with 3-phase power supply.

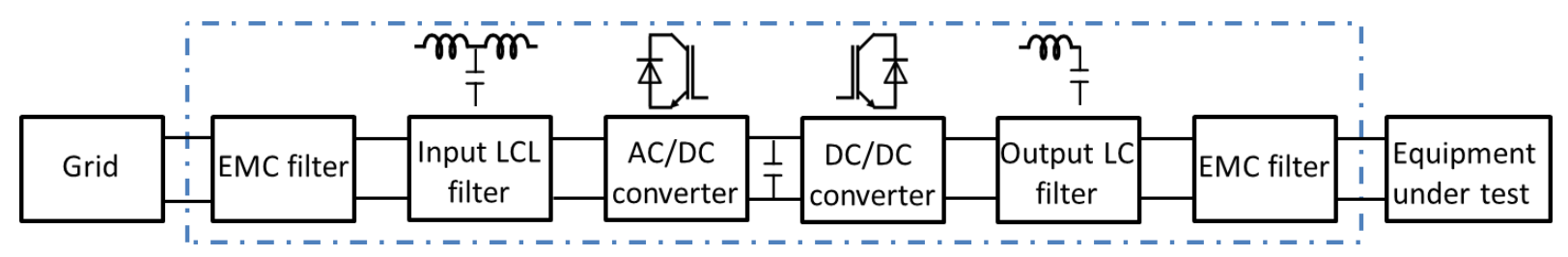

Figure 4.2 CINERGIA B2C30 equipment back-to-back topology

A back-to-back topology of the converter's hardware is illustrated in figure 4.2 [97]. CINERGIA B2C30 equipment uses in both ends EMC filters. Those filters are used for reducing in between mains of power supply and the drives, the transfer of electromagnetic noise. After the EMC filter in the grid side of the topology, an LCL filter is applied. The scope of this filter is reducing total harmonic distortion and current distortion on higher or equal frequencies of switching frequency. The next box shows an AC/DC converter. This converter is composed of three-branch insulated-gate bipolar transistor IGBT active rectifier with bidirectional power flow. This rectifier controls the dc link voltage and at the same time exchanges sinusoidal current from or to the grid. Next part of the topology is a three-branch IGBT DC-DC converter also with bidirectional power flow ability regulating the output, in this case the dc current. Last part of the topology is an LC filter which is used to reduce the current distortion formed by switching, at the output of the circuit.

The open cabinet of the CINERGIA B2C30 equipment is shown in figure 4.3. In the figure are indicated 9 different features of the equipment. Point 1 is showing the $A C$ input breaker while the point 2 the converter output to the equipment under test. Points 8 and 9 are shown the earth protection points.

Points 3 and 4 are indicating the four operation modes of the dc output. At point 3 independent and parallel modes are in place 0 and 1, respectively. Likewise, at point 4 bipolar is placed in 0 and unipolar in 1. As it was mentioned, the type of experiments which will be implemented categorised to independent unipolar mode. Therefore, the switch at point 3 is pointing at 0 and point 4 at 1. 


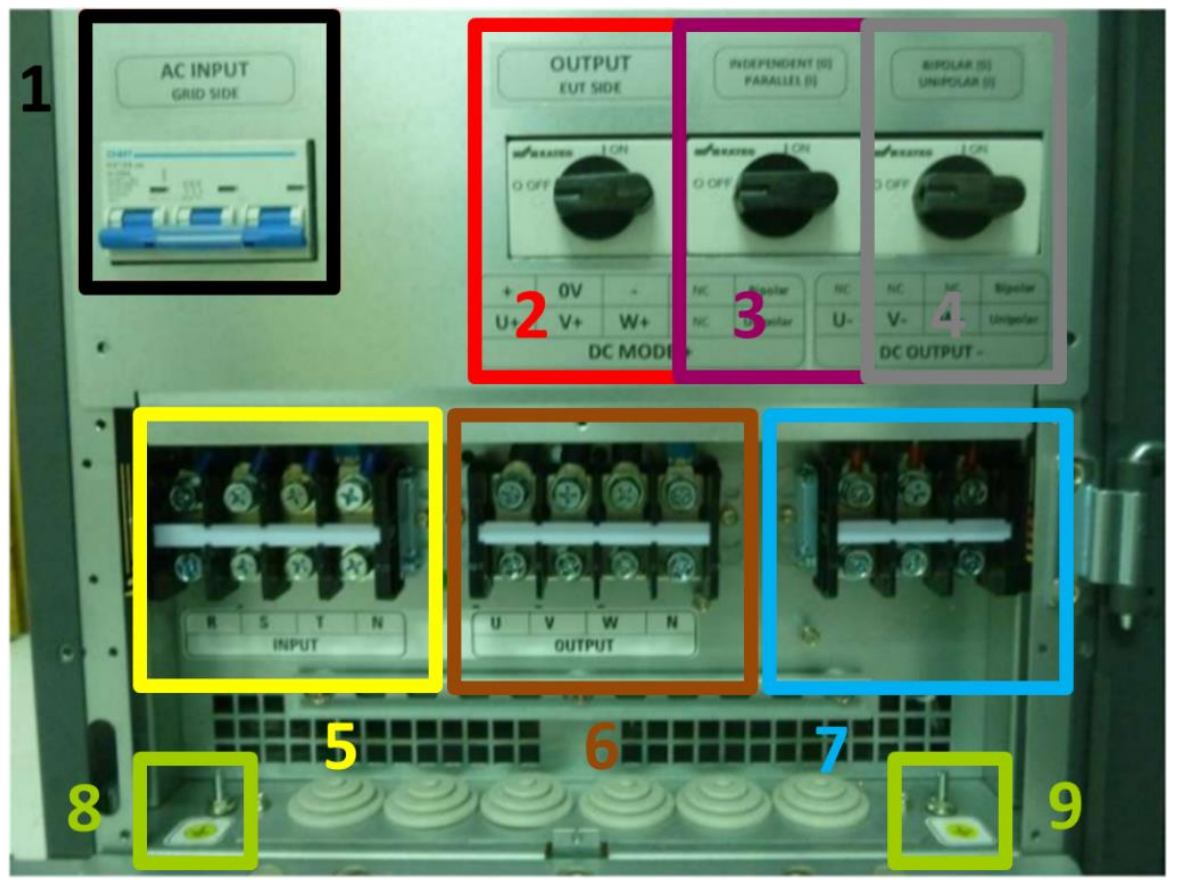

Figure 4.3 Open CINERGIA B2C30 cabinet displaying different features of the equipment

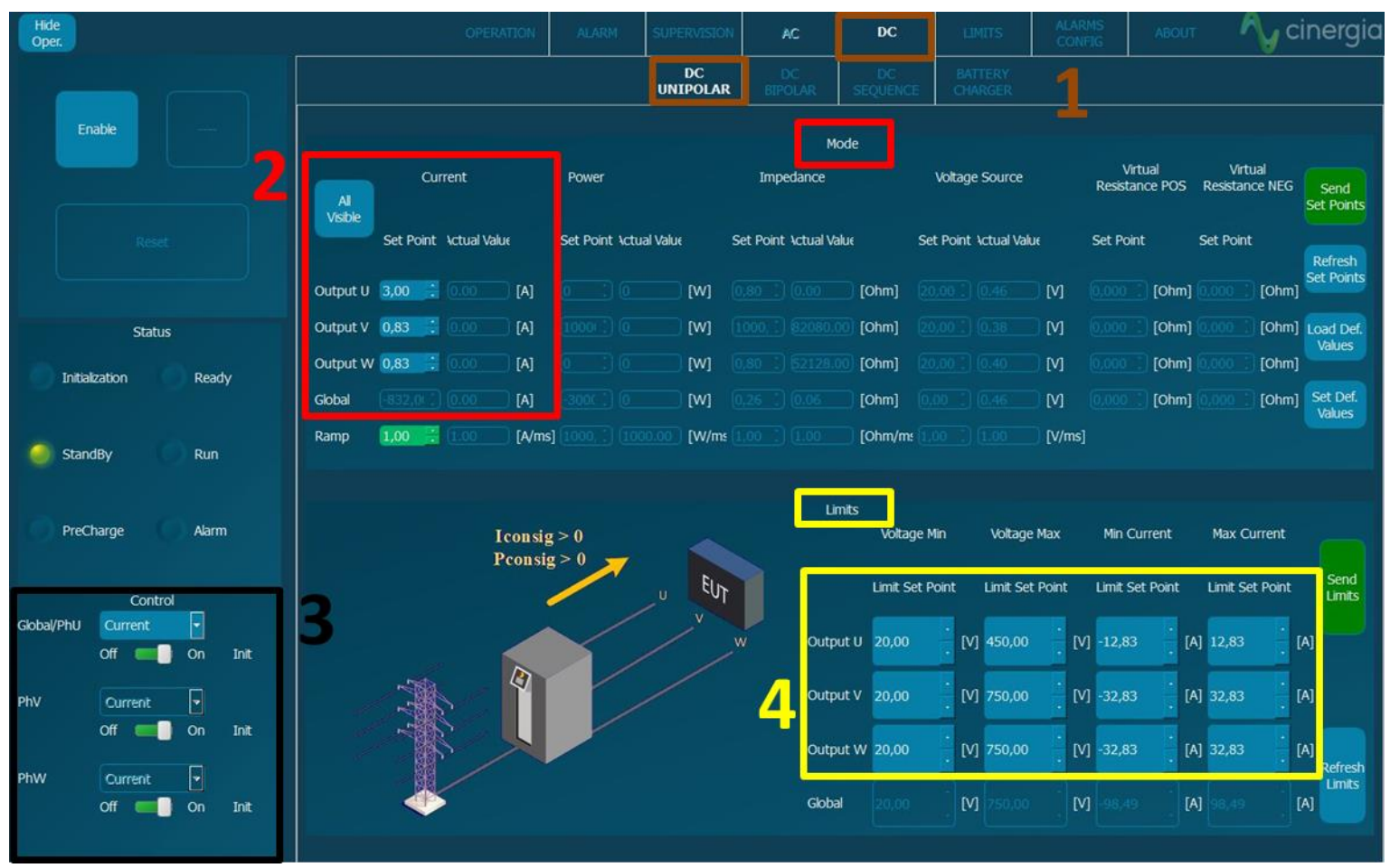

Figure 4.4 CINERGIA B2C30 human machine interface control 
Point 5 is showing the three-phase grid input of the system and 6 and 7 points the dc side. At point 5 , three phases along with neutral cable will be connected while in 6 and 7 the connection is depended on the connection mode. In this case as it shown in figure 4.3, there are three channels of output. Each channel has their positive and negative which is connected respectively to the equipment under test (source or load). The positive side of the three output channels is shown at point 6 with negative at point 7. In case of using only one output the positive cable would be connected at the entry $U$ of point 6 and the negative in the first entry (from the left) of point 7.

Finally, the human machine interface of the CINERGIA B2C30 is shown in figure 4.4. Using the control panel, the DC constant current operation mode is selected among the four possible operating modes (constant impedance, constant current, constant voltage and constant power). The equipment includes protections and the DC output voltage is limited to $450 \mathrm{~V}$, the $A C$ current to $10 \mathrm{~A}$. This limitation of the $A C$ current implies a limitation in the DC current between $-12.83 \mathrm{~A}$ to $+12.83 \mathrm{~A}$.

\subsection{Overview of the setup 1}

In the first setup, the CINERGIA B2C30 Power Converter is used as Bidirectional AC/DC Power converter able to behave as a bidirectional battery charger, but the battery is replaced by a resistor in G2V operating mode and by a DC Power Source in V2G operating mode. These modifications look for a more precise control during the required tests to identify the model's transfer functions. In practice, the proposed method to generate the black-box behavioural model is valid, not only for battery chargers, it is also valid for any AC/DC converter, either unidirectional or bidirectional.

The first setup is consisted of the CINERGIA B2C30 equipment, a unidirectional power supply (Sorensen DHP series 400V, 37A) [98] and a programmable DC electronic load (Chroma model 63200) [99] operating in constant resistive mode. This setup is dealing with two types of experiments, the G2V and V2G. As it was mentioned before in this chapter the AC side of CINERGIA B2C30 equipment is connected with a three-phase plug. However, in the current experiments data only from one phase will be collected. Also, in the DC output part of CINERGIA B2C30 equipment only the $U$ channel will be connected with the respective minus slot.

In figure 4.5 the connection diagram of a G2V mode is shown. In order to create a G2V system two parts are needed, a battery charger (CINERGIA B2C30 equipment) operates in current mode and a load (Chroma) in resistive mode replacing the charging mode of a real battery by a resistor.

In figure 4.6 a diagram with a V2G connection mode is shown. In this case -since a real does not exist- in the current G2V setup a unidirectional DC power supply (Sorensen DHP series) enters and substitutes the battery. The procedure of 'discharging' the battery is by using the CINERGIA B2C30 equipment once more as current source but setting the DC current to a negative amount. Likewise, a negative power flow is safely achieved. 


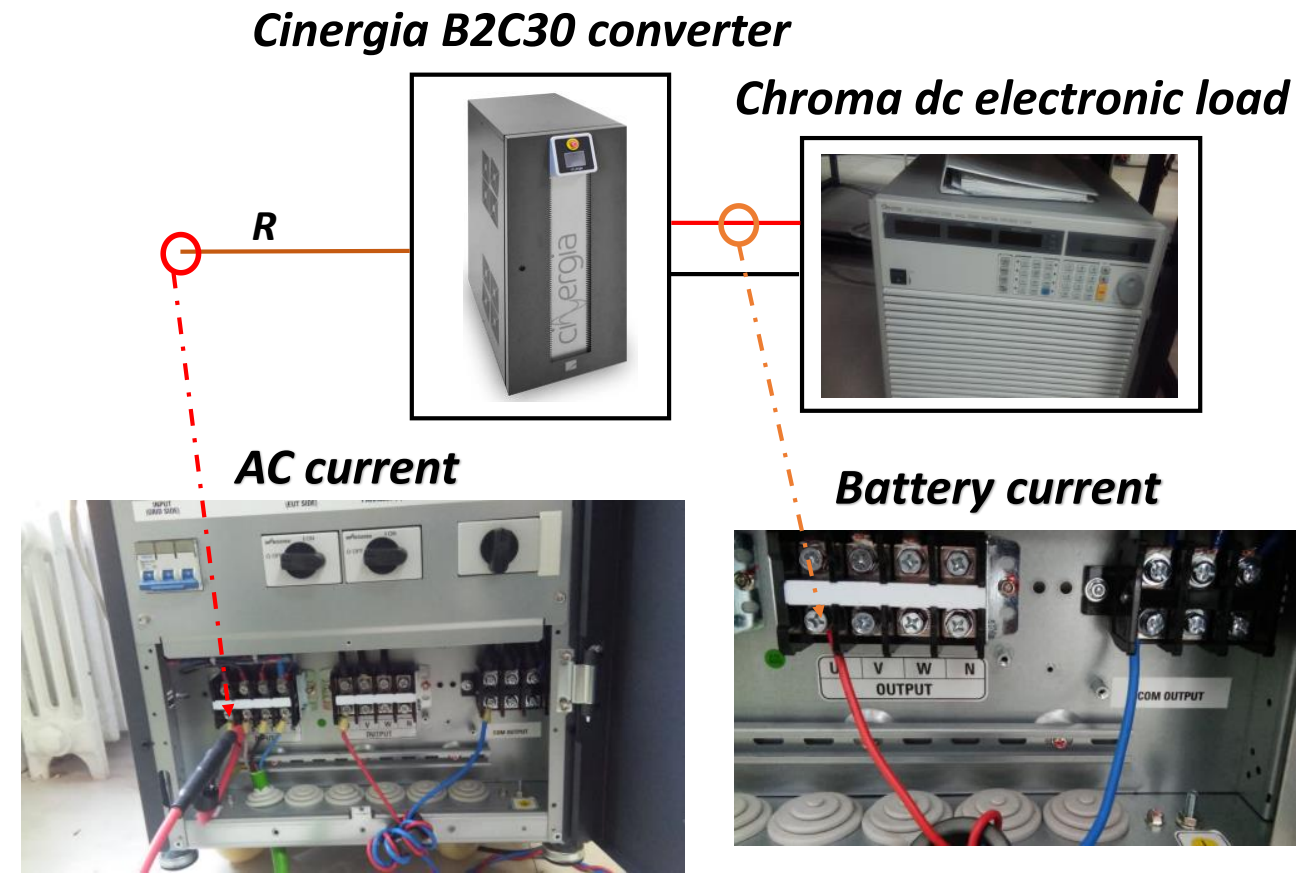

Figure 4.5 Grid to Vehicle mode connection

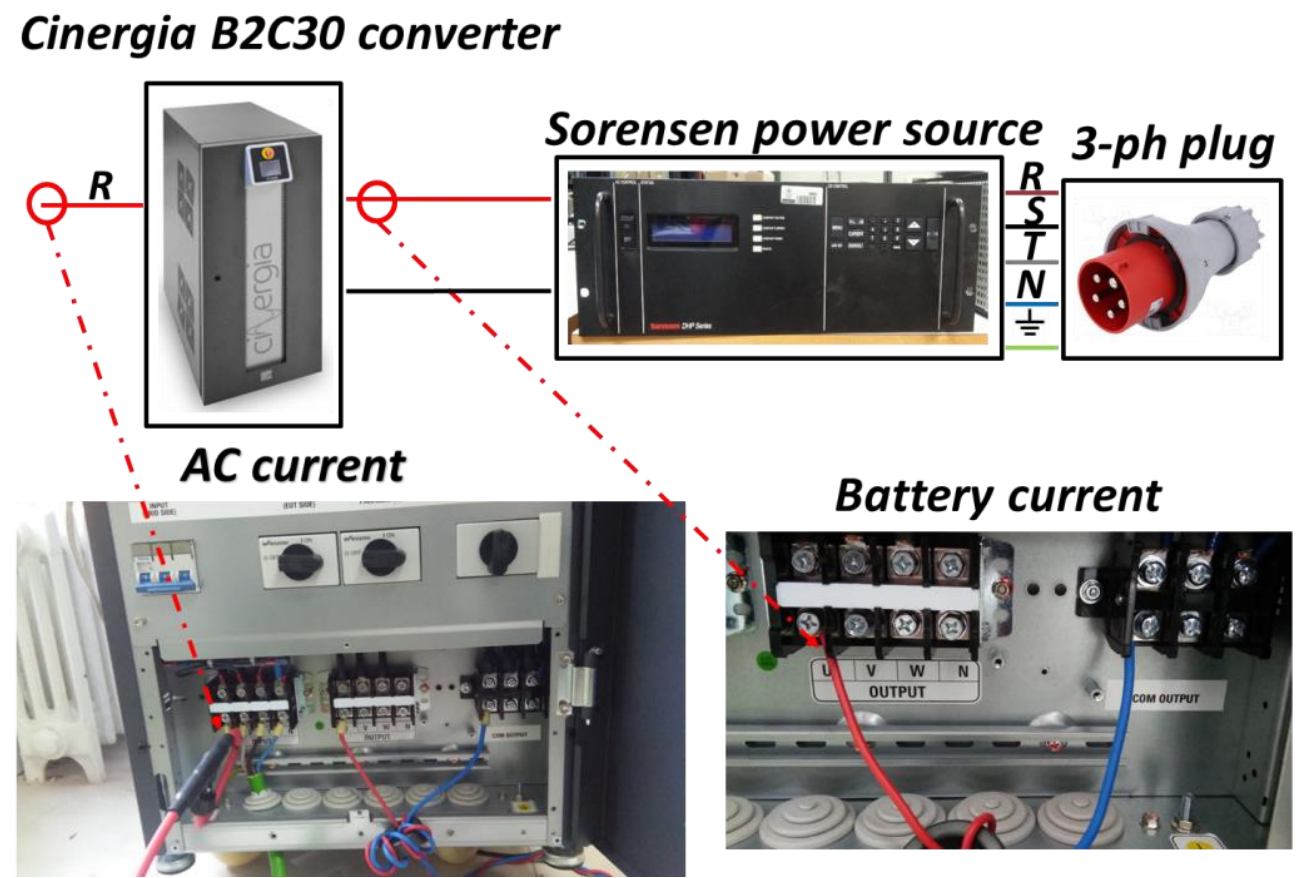

Figure 4.6 Vehicle to Grid mode connection 


\subsection{Black-box model for experimental setup 1}

Once the setup 1 has been installed, next step deals with the identification of the transfer functions of the battery chargers. In chapter 3 the identification process was based on the data depicted from PSIM program, but now in the next 2 black-box sections will show the identification process of experimental setup not only assuming small-signal conditions, but also large-signal. The data on these sections will be taken directly from the equipment setup as it is shown in figure 4.7.

It is necessary to mentioned that even with a 3-phase connection plug, the results will show only the aphase. Likewise, in the output, only the a-phase of the 3-ph current is shown in the results.

Figure 4.8 is representing the battery charger converter in a black-box diagram. The input variables of $A C / D C$ Power converter are the 3-phase input voltages $\left(V_{a}, V_{b}, V_{c}\right)$ along with reference current control in the DC part ( Iref $_{\text {f }}$. Both signals have to enter the black-box model in DC values. The 3-phase input voltages are transformed to $d$-q framework. A single Park transformation box is needed to get the abc values to dq. Once the values are transformed to $d q$, are being subtracted from their respective nominal values $\left(V_{d}{ }^{d c}, V_{q}{ }^{d c}\right)$ ending up with their perturbation values $\left(\hat{v}_{d}, \hat{v}_{q}\right)$ entering the black-box model. In case of $I_{\text {ref }}$ value the subtraction from the nominal respective value $\left(I_{\text {ref }}{ }^{d c}\right)$ it provides with the perturbation input value $\left(\hat{l}_{\text {ref }}\right)$.

On the output side of the black-box model, the perturbation signals from the dq framework $\left(\hat{l}_{\mathrm{d}}, \hat{l}_{\mathrm{q}}\right)$ are added with the respective nominal values $\left(I_{d}{ }^{d c}, I_{q}{ }^{d c}\right)$ and with an inverse park transformation the 3-phase

\section{Cinergia B2C30 converter}

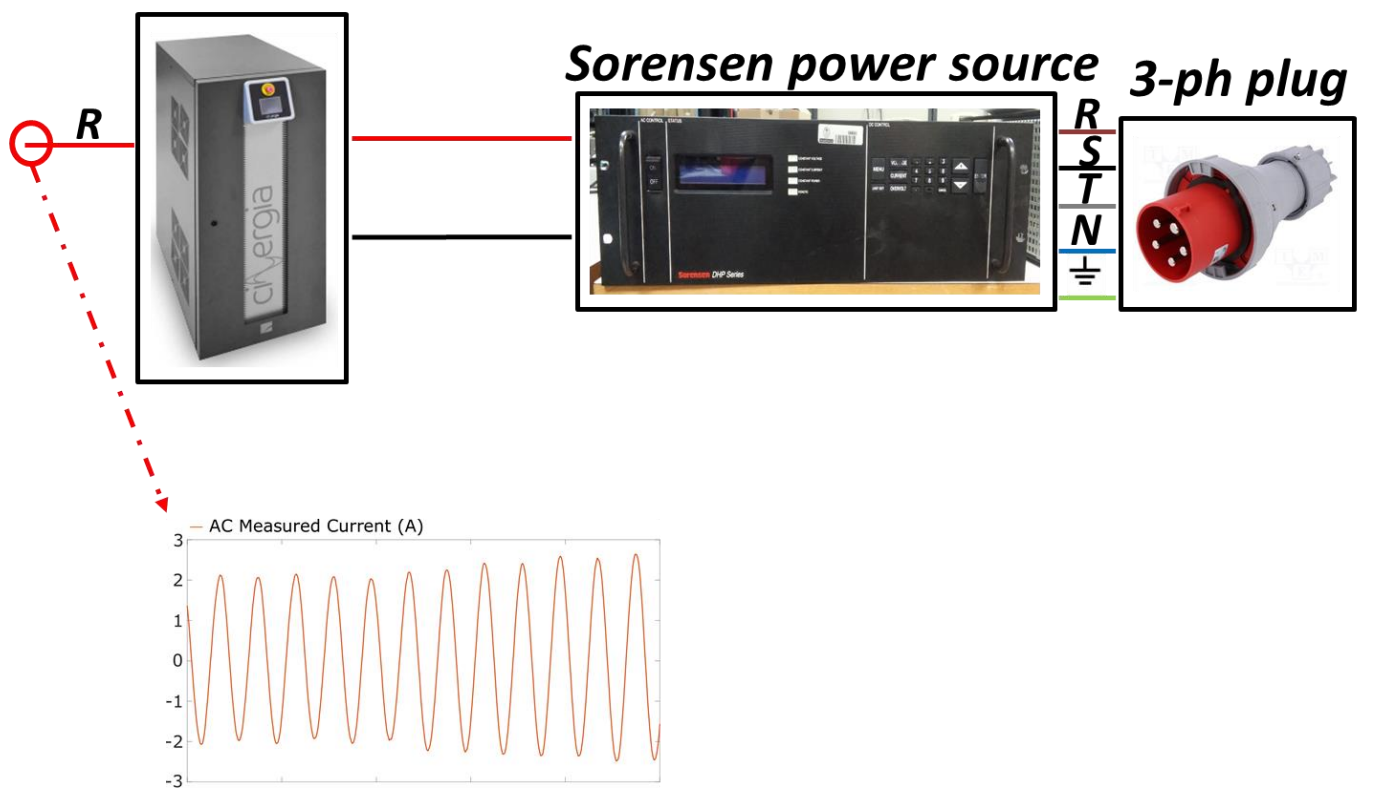

Figure 4.7 Experimental setup 1 in V2G operating mode 


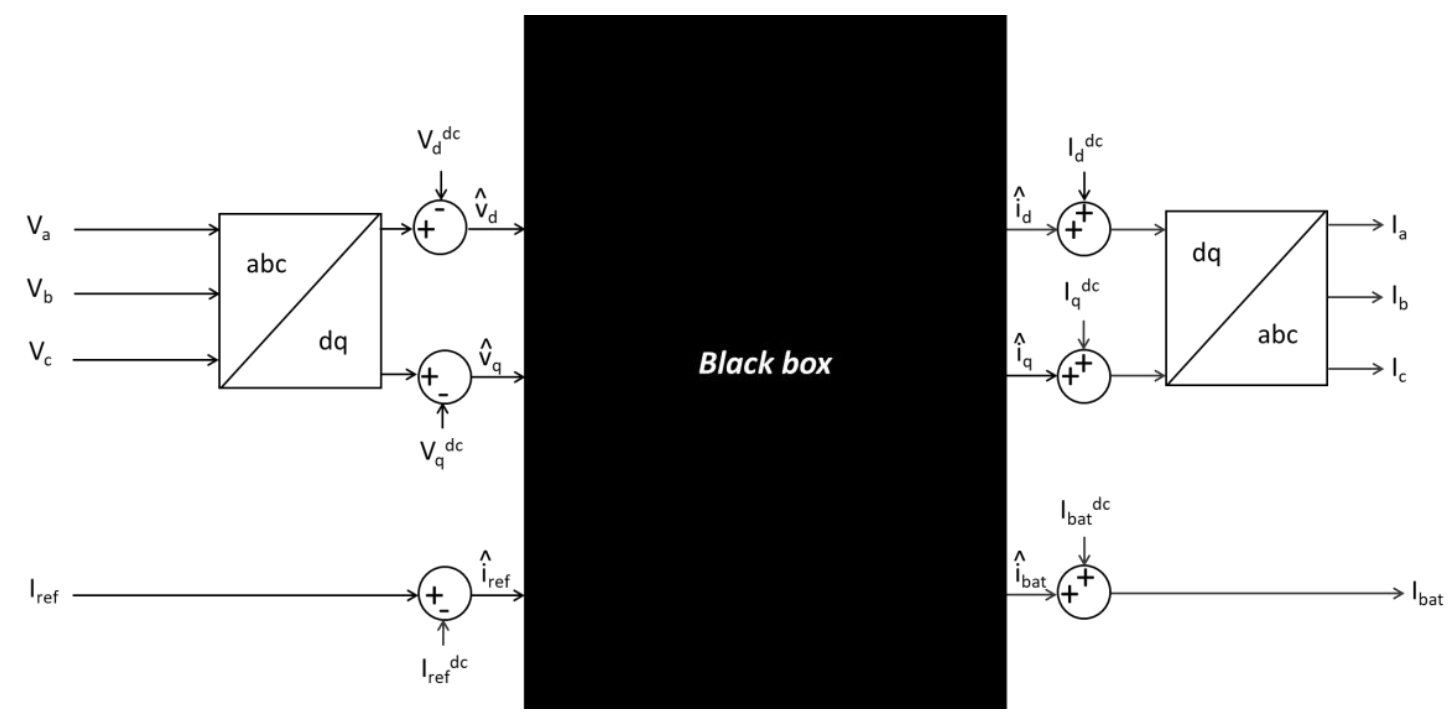

Figure 4.8 Block diagram for the small-signal black-box model for the experimental setup 1

output variables $\left(I_{a}, I_{b}, I_{c}\right)$ are obtained. The battery current output $\left(I_{b a t}\right)$ is obtained by the addition of the current perturbation signal $\left(\hat{l}_{\text {bat }}\right)$ and the respective nominal value $\left(l_{\text {bat }}{ }^{d c}\right)$. Assuming small-signal conditions, that implies the system can be considered linear, then perturbations in each input are applied one input at a time resulting on the output perturbation signals. Each input is affecting with a perturbation 3 different outputs. Therefor with 3 inputs the dynamic responses have 9 transfer functions.

This small-signal model described in chapter 2 and checked by virtual prototyping in chapter 3 is again represented by the following matrix (E 4.1) the input and output perturbations taken from the figure 4.8 are shown. The output perturbations $\left(\hat{l}_{d}, \hat{l}_{q}, \hat{l}_{\text {bat }}\right)$ are shown on the left side of the matrix and the inputs $\left(\hat{v}_{\mathrm{d}}, \hat{v}_{\mathrm{q}}, \hat{\imath}_{\mathrm{ref}}\right)$ on the right side. The nine transfer functions obtained are represented by $\mathrm{Y}_{\mathrm{dd}}, \mathrm{Y}_{\mathrm{dq}}, \mathrm{H}_{\mathrm{dref}}, \mathrm{Y}_{\mathrm{qd}}$, $\mathrm{Y}_{\text {qq }}, \mathrm{H}_{\text {qref, }} \mathrm{Y}_{\text {batd }}, \mathrm{H}_{\text {batref. }}$

$$
\left(\begin{array}{c}
\hat{i}_{d}(s) \\
\hat{i}_{q}(s) \\
\hat{i}_{\text {bat }}(s)
\end{array}\right)=\left(\begin{array}{ccc}
Y_{d d}(s) & Y_{d q}(s) & H_{\text {dref }}(s) \\
Y_{q d}(s) & Y_{q q}(s) & H_{\text {qref }}(s) \\
Y_{\text {batd }}(s) & Y_{\text {batq }}(s) & H_{\text {batref }}(s)
\end{array}\right) \cdot\left(\begin{array}{c}
\hat{v}_{d}(s) \\
\hat{v}_{q}(s) \\
\hat{i}_{\text {ref }}(s)
\end{array}\right)
$$


The small-signal black-box model represented by equation $\mathrm{E} 4.1$ may be also represented by the block diagram of figure 4.9 and the equivalent circuit of figure 4.10 .

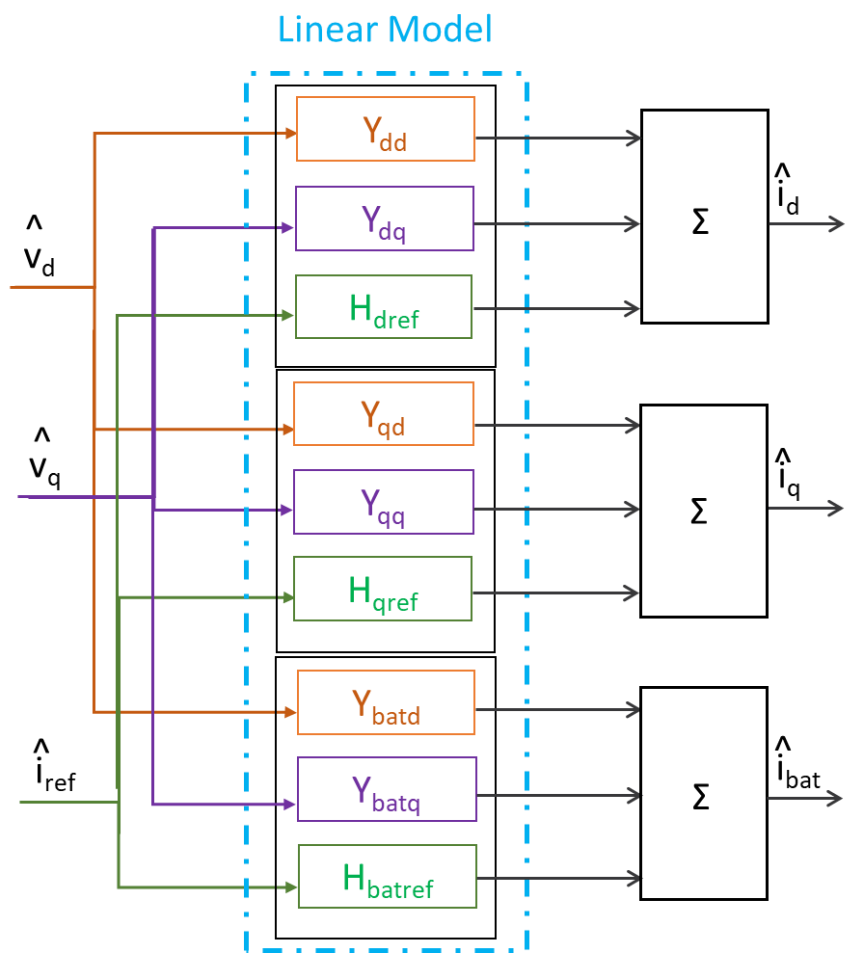

Figure 4.9 Identified transfer functions for the small-signal model in experimental setup 1

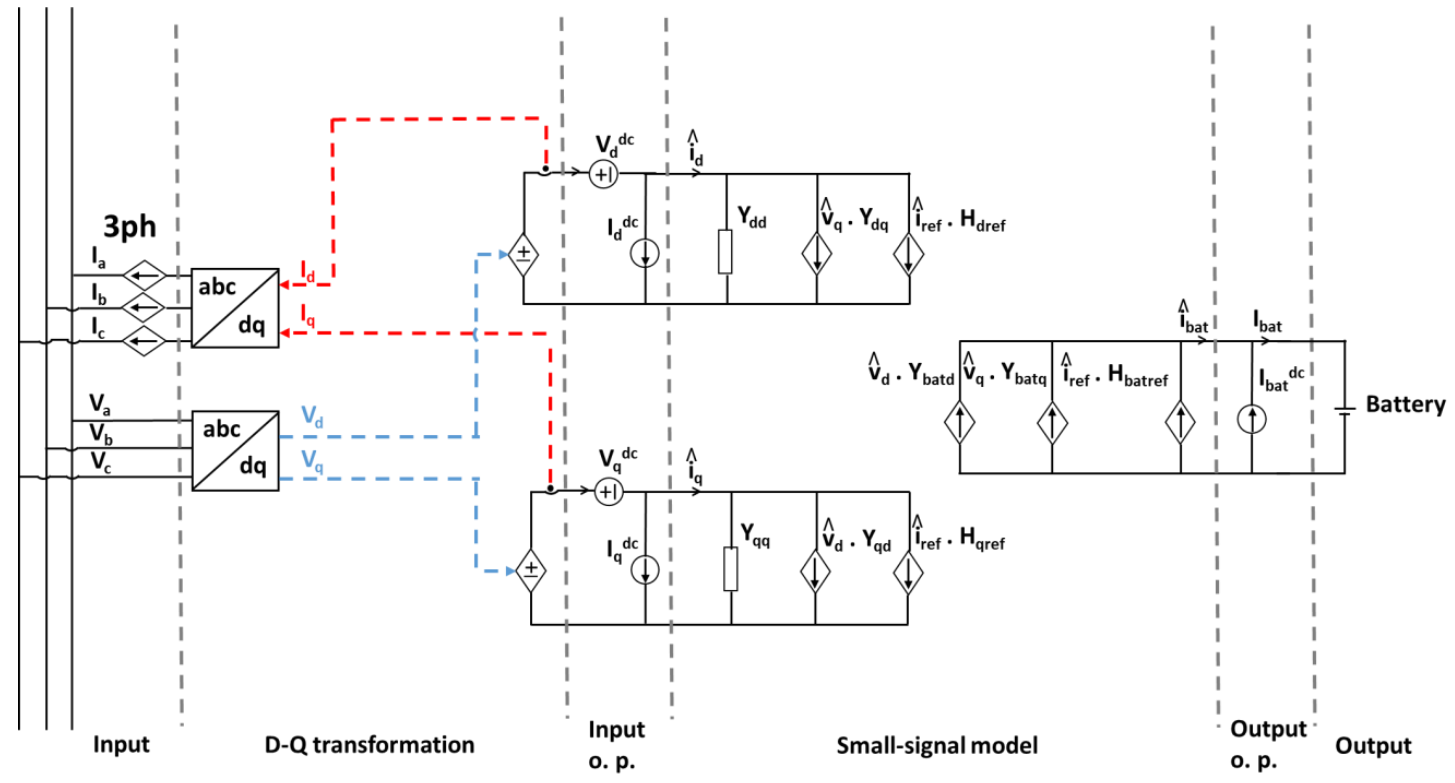

Figure 4.10 Equivalent circuit of the small-signal black-box model of experimental setup 1 


\subsubsection{Comparison of small-signal black-box model and experimental setup 1}

Following the previous examples of virtual prototyping in chapter 3 , the results which will be compared in this subsection are from the experimental setup 1 and will be compared to black-box models. First, we consider that small-signal conditions are met and the comparison between the small-signal model and the actual equipment in setup 1 will described in this section.

The 3 tests are two operating in $G 2 \mathrm{~V}$ mode in different power change ( $3 \mathrm{~A}$ to $4 \mathrm{~A}$ and $3 \mathrm{~A}$ to $8 \mathrm{~A}$ ) and one in V2G mode (-3A to $-9 A)$. This means that the initial operating point corresponds to a DC current of $3 A$ in G2V operation and -3 A in V2G operation. On the other hand, to simplify the experiments, the 3-phase AC voltage is kept constant along the tests. In G2V mode the resistive load in all cases from Chroma electronic load was set to $80 \Omega$. In this comparison the focus is put on the estimation of AC current.

3-phase AC current is only affected by the transfer functions $H_{\text {dref }}$ and $H_{\text {qref }}$ (figure 4.9). In this set of experiments the transfer functions have been created by using polynomial discrete method with output error.

The first model representation is the test with a perturbation of the reference of the DC current from $3 \mathrm{~A}$ to $4 \mathrm{~A}$. This means a perturbation in the reference current control $\left(\mathrm{I}_{\text {ref }}\right)$ positive $1 \mathrm{~A}$ from a steady state $3 \mathrm{~A}$. The identified functions created for the black-box modelling are listed in the table 4.1 and their corresponding mathematical expressions.

In figure 4.11 the first test is shown with the upper figure showing the AC current comparison and followed by its $d$-q components. The measuring results in all 3 -fold figure here, can be seen in orange colour while the black-box model in blue. The time of capture this test observed the current change from $3 \mathrm{~A}$ to $4 \mathrm{~A}$ is happening at $0.27 \mathrm{~s}$.

\begin{tabular}{|c|c|c|c|}
\hline Tests & Iref Step & $\begin{array}{c}\text { Transfer } \\
\text { Functions }\end{array}$ & Identified Transfer Functions \\
\hline \multirow{2}{*}{ Test1 } & \multirow{3}{*}{$3 \mathrm{~A} \rightarrow 4 \mathrm{~A}$} & $H_{\text {dref }}(z)$ & $\frac{2.94 e-5 z-2.938 e-5}{z^{2}-2 z+1}$ \\
\cline { 3 - 4 } & & $H_{\text {qref }}(z)$ & $\frac{0.9962 e-3 z^{3}+0.007099 z^{2}-0.01718 z+0.009089}{z^{4}-2 z^{3}+z^{2}}$ \\
\hline
\end{tabular}

Table 4.1 Identified transfer functions from the small-signal model in experimental setup 1

In the second test of $5 \mathrm{~A}$ step the same transfer functions obtained in the previous test of $1 \mathrm{~A}$ (with $3 \mathrm{~A}$ initial point) are used. This test is made to check the accuracy of the small-signal model in case of large perturbations in the battery current reference. As it can be observed easily in the figure 4.12, the blackbox model (in blue colour) which created does not coincide with the measured results (orange colour). In this figure there is huge area for improvement. In this condition the small-signal model is not valid anymore, therefor a nonlinear solution must be applied. 

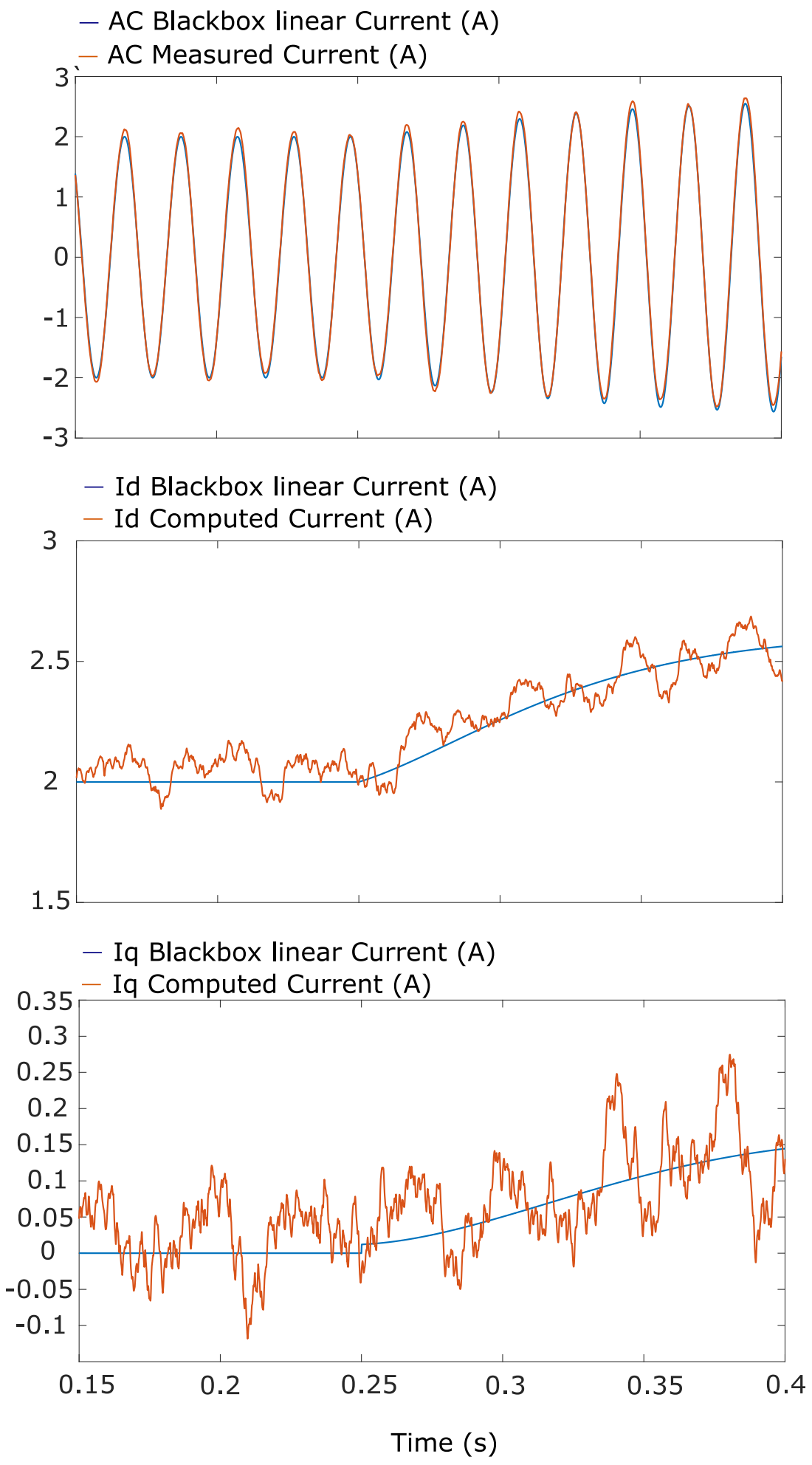

Figure 4.11 Results comparison between switching and black-box models for AC current response (upper) with $\mathrm{d}$-q components in a $1 \mathrm{~A}$ step test in $\mathrm{G} 2 \mathrm{~V}$ mode 


\subsubsection{Non-linear polytopic model}

In small signal operation, a black-box model with the linear method described above is adequate. Although as it applies larger steps the dynamic behaviour starts to change and does not follows the actual converter's path. The reason for this lack of accuracy depends on the space between the operating point of the converter and the step applied. This behaviour requires a different approach to model the converter's behaviour (figure 4.13).

According to the previous information about the linear small-signal, in case of nonlinearity presence, a nonlinear method known as polytopic is taking place. This concept was presented in Chapter 2 and it is one of the main contributions of this thesis.

\section{- AC Blackbox linear Current (A)}

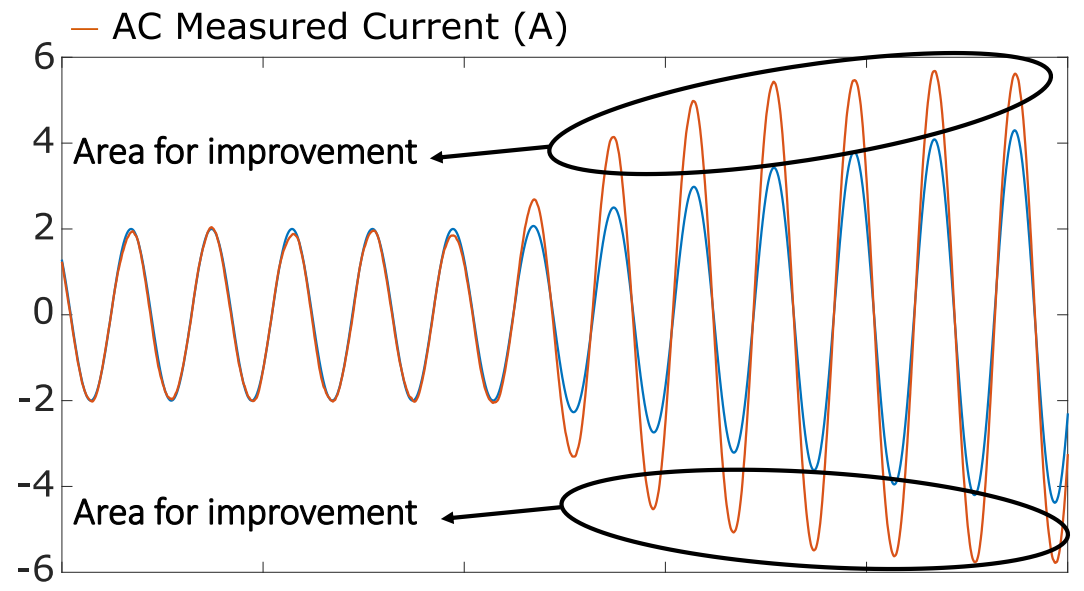

Figure 4.12 Results comparison between switching and small-signal black-box model method for AC current response (upper) with $d$-q components in a $5 \mathrm{~A}$ step test in $\mathrm{G} 2 \mathrm{~V}$ mode

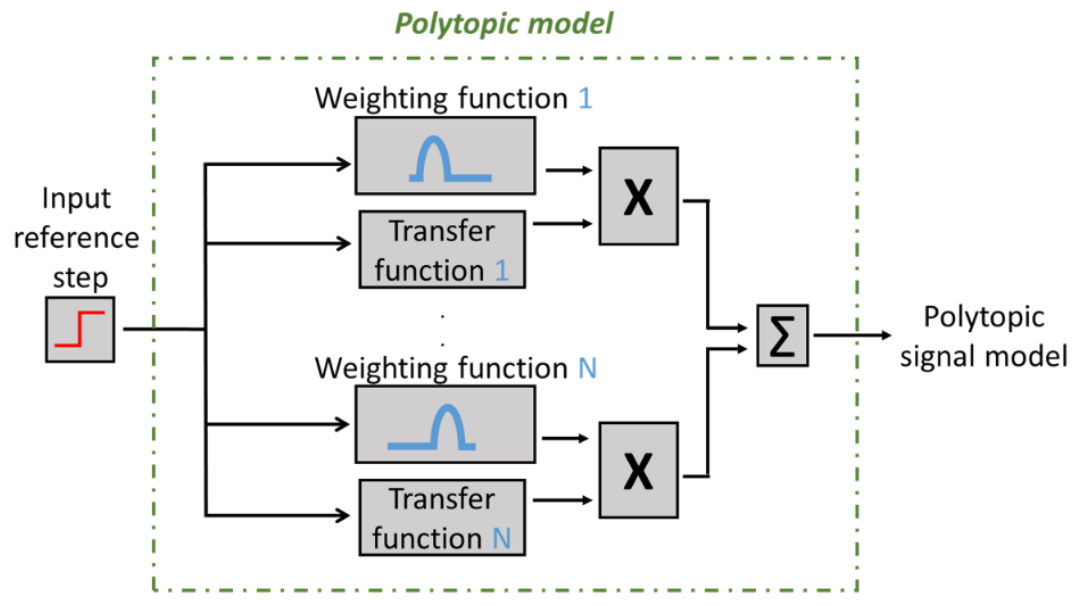

Figure 4.13 Polytopic nonlinear approach 
In fact, a large-signal black-box model for bidirectional AC/DC converters is proposed, and this modelling strategy is particularly applied to bidirectional battery chargers.

As it was explained in Chapter 2, the idea behind the polytopic approach is a group of local (linear) models in different operating points combined with nonlinear weighting functions operating as a single nonlinear structure. Although by using the polytopic model to cover the insufficient linear method, must be taken into consideration the accuracy of the system and avoid being excessively complex.

The accurate selection of local models in combination with the correct selection of weighting functions (W.F.) is vital. A combination transfer and weighting functions is shown in figure 4.14. From previous work applied to modelling power converters, the Double sigmoid function (E 4.2) is showed to be the most suitable weighting function for this application. It is well known; that the weighting functions summation must be equal to 1 while their range from 0 to 1 (figure 4.15).

Experimentation with the specific function has shown that by weighted their segments 50:50, the points of inflexions are exactly in the middle and then the accuracy is at the maximum. Also, the parameters affecting the double sigmoid depends on the slope (parameter a in the equation) depending on the power change and the point of the bend inflexion (parameter $c$ in the equation). An example of the slope of the bend it can be seen in figure 4.16. On the left side of the figure 4.16 a summation of linear models recreating a bend for a nonlinear model and likewise on the right side of the figure 4.16 shows the same procedure of a pond recreated by a number of small drops put together.

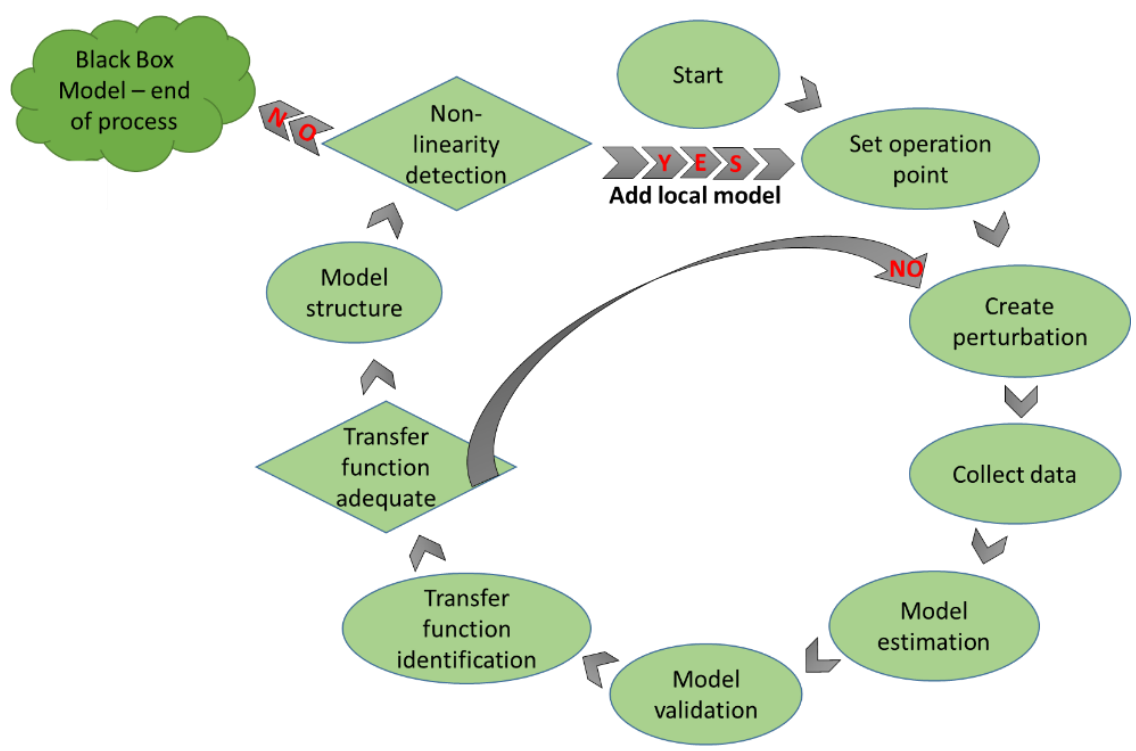

Figure 4.14 Polytopic model example of transfer and weighting functions combination 


$$
F(t)=\frac{1}{1+e^{-a(x-c)}}
$$

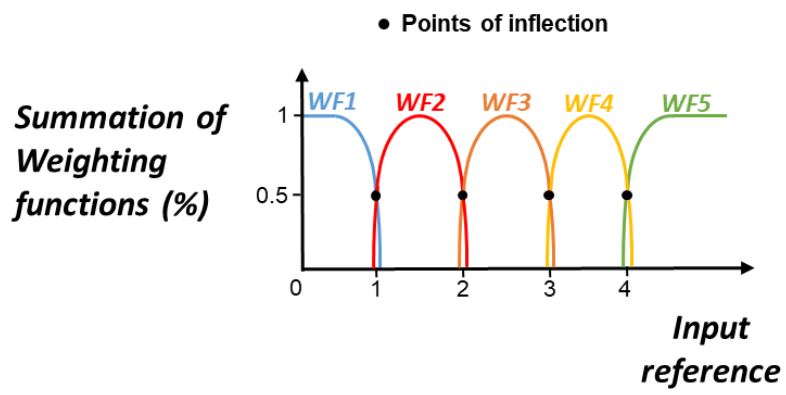

Figure 4.15 Block diagram of double sigmoid weighting functions
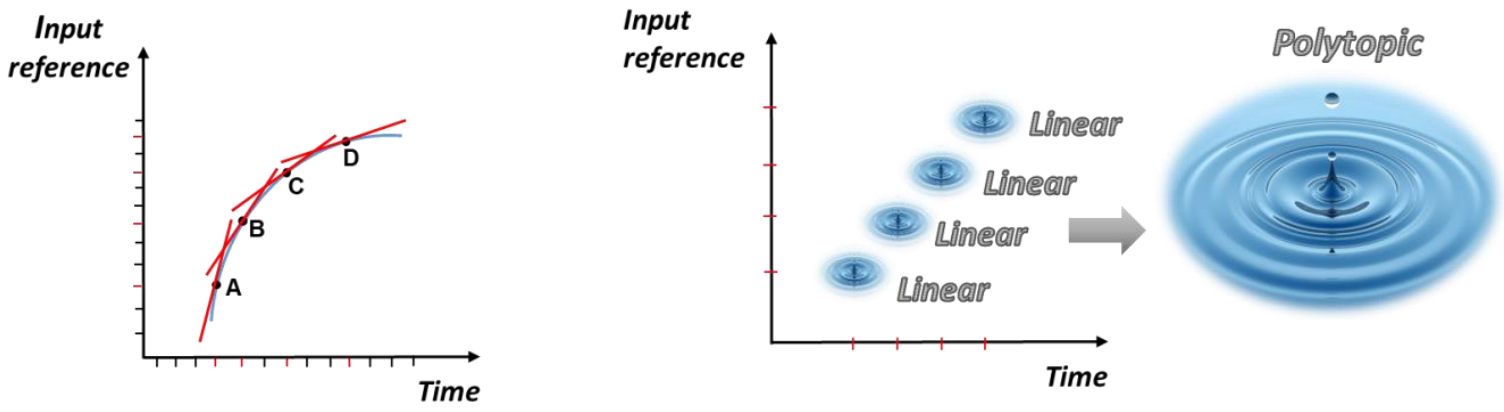

Figure 4.16 Summation of linear models recreating a bend for a nonlinear model (left), Real life application of polytopic model (right)

\subsubsection{Comparison of black-box polytopic model with experimental setup 1}

If we consider the same experiment with a large step (5A) in the DC current reference, starting from an initial value of $3 \mathrm{~A}$, as it was checked in the previous section with the small-signal model, but now with the large-signal polytopic model. In this case, three different linear models were created at three different operating points (3A, $4 \mathrm{~A}$ and $5 \mathrm{~A})$. These models were obtained following the same procedure as in the small-signal section. Again, it is assumed than the 3-phase remains constant and a $1 \mathrm{~A}$ step is given in the DC current reference to obtain the model transfer functions keeping the small-signal conditions. Finally, these three models are combined with the Double Sigmoid weighting functions. The calculated model's transfer functions are indicated in Table 4.2

The large-signal model is tested and compared with the actual AC/DC converter in the setup 1 and the results are shown in figure 4.17 (large-signal model in blue colour, measured results in orange colour). 
Results show an excellent accuracy being difficult to distinguish the measured AC current from the AC current generated by the large-signal model. These results show a clear improvement representing the converter behaviour with large-signal perturbations. Although the computation time is higher, the number of tests to obtain the data to generate the model is clearly three times that the number of tests required for the linear model, the improvement in terms of accuracy justify the extra complexity in the modelling strategy. Similar results have been obtained previously in DC/DC converters and DC/AC converters. This extension of the applicability of Polytopic model to AC/DC converters is one of the main contributions of this thesis.

Finally, the V2G operating mode is also considered, in case of large-signal perturbations. The converter is operating with -3A DC current and a large-signal step in the DC current reference is given from -3A to -9A. Negatives values in the DC current mean that the power flow goes from the DC side to the $A C$ side. In the setup 1 , in the V2G mode the battery is replaced by a DC Voltage Source set to $400 \mathrm{~V}$. At $t=0.17 \mathrm{~s}$ a negative step of $6 \mathrm{~A}$ is applied. In this case to obtain the large-signal polytopic model, for small-signal model are generated in around four different values of the $D C$ current reference $(-3 A,-4 A,-5 A,-6 A)$, giving a step of $-1 \mathrm{~A}$ in the current reference to keep the small-signal conditions. This situation is shown in Table 4.3 with transfer functions obtained for the four operating points. Once the large-signal model is generated, the comparison between the measurements and the model output is represented in figure 4.18. for the AC current. The negative step of $-6 \mathrm{~A}$ is applied at $\mathrm{t}=0.17 \mathrm{~s}$, both results are so close being difficult to distinguish both waveforms. In the figure the AC voltage is also represented to show the converter is operating in the V2G mode.

\begin{tabular}{|c|c|c|c|}
\hline Tests & $\begin{array}{l}\text { Transfer } \\
\text { Functions }\end{array}$ & Iref Steps & Identified Transfer Functions \\
\hline \multirow{6}{*}{ Test 1} & \multirow{3}{*}{$H_{\text {dref }}(z)$} & $3 \mathrm{~A} \rightarrow 4 \mathrm{~A}$ & $\frac{2.94 e-5 z-2.938 e-5}{z^{2}-2 z+1}$ \\
\hline & & $4 \mathrm{~A} \rightarrow 5 \mathrm{~A}$ & $\frac{1.22 e-3 z^{2}+1.656 e-5 z-1.203 e-3}{z^{3}-0.9281 z^{2}-z+0.9281}$ \\
\hline & & $5 \mathrm{~A} \rightarrow 6 \mathrm{~A}$ & $\frac{-0.1908 e-3 z^{2}+0.386 e-3 z-0.1952 e-3}{z^{3}-2.993 z^{2}+2.986 z-0.9932}$ \\
\hline & \multirow{3}{*}{$H_{\text {qref }}(z)$} & $3 \mathrm{~A} \rightarrow 4 \mathrm{~A}$ & $\frac{0.9962 e-3 z^{3}+0.007099 z^{2}-0.01718 z+0.009089}{z^{4}-2 z^{3}+z^{2}}$ \\
\hline & & $4 \mathrm{~A} \rightarrow 5 \mathrm{~A}$ & $\frac{-8.311 e-5 z-8.316 e-5}{z^{2}-1.999 z+0.9985}$ \\
\hline & & $5 \mathrm{~A} \rightarrow 6 \mathrm{~A}$ & $\frac{-0.267 e-3 z^{2}+2.456 e-5 z+0.293 e-3}{z^{3}-0.9117 z^{2}-z+0.9119}$ \\
\hline
\end{tabular}

Table 4.2 Identified transfer functions for G2V operation in z-domain. 
- AC Blackbox polytopic Current (A)

- AC Measured Current (A)
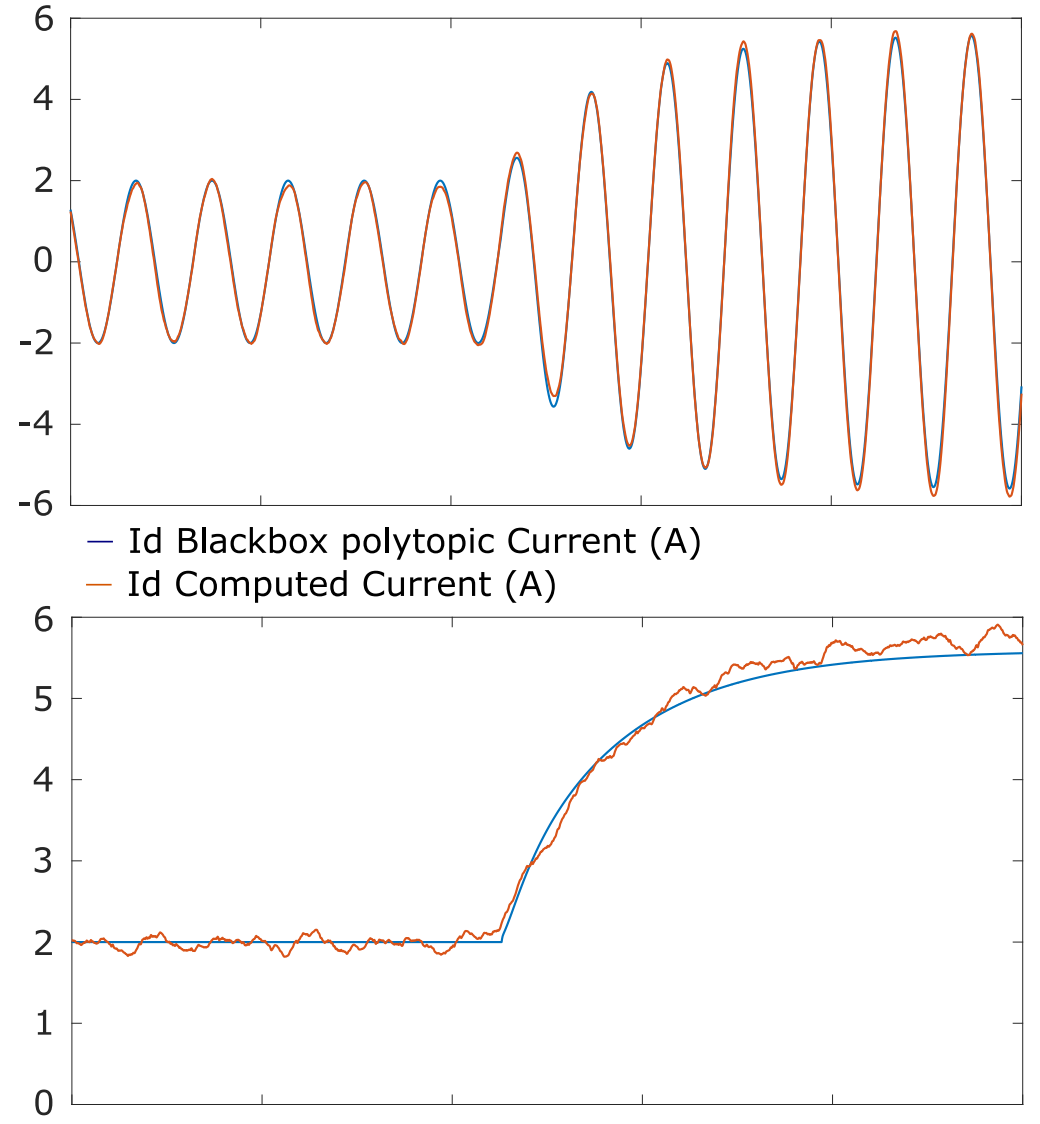

- Iq Blackbox polytopic Current (A)

- Iq Computed Current (A)

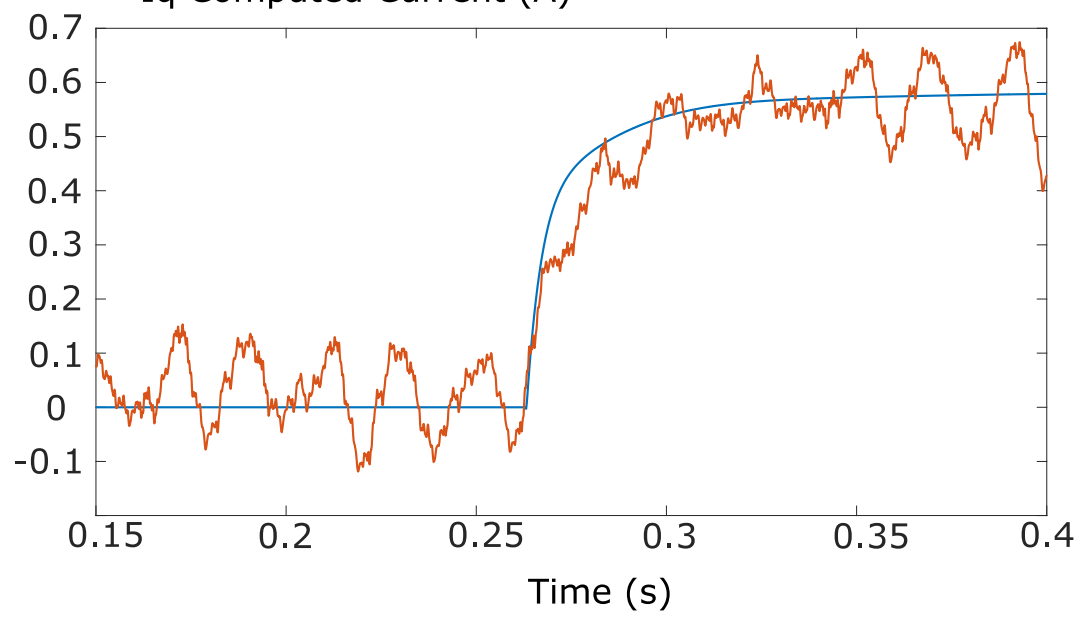

Figure 4.17 Results comparison between switching and polytopic black-box model method for AC current response (upper) with d-q components in a 5A step test in $\mathrm{G} 2 \mathrm{~V}$ mode 


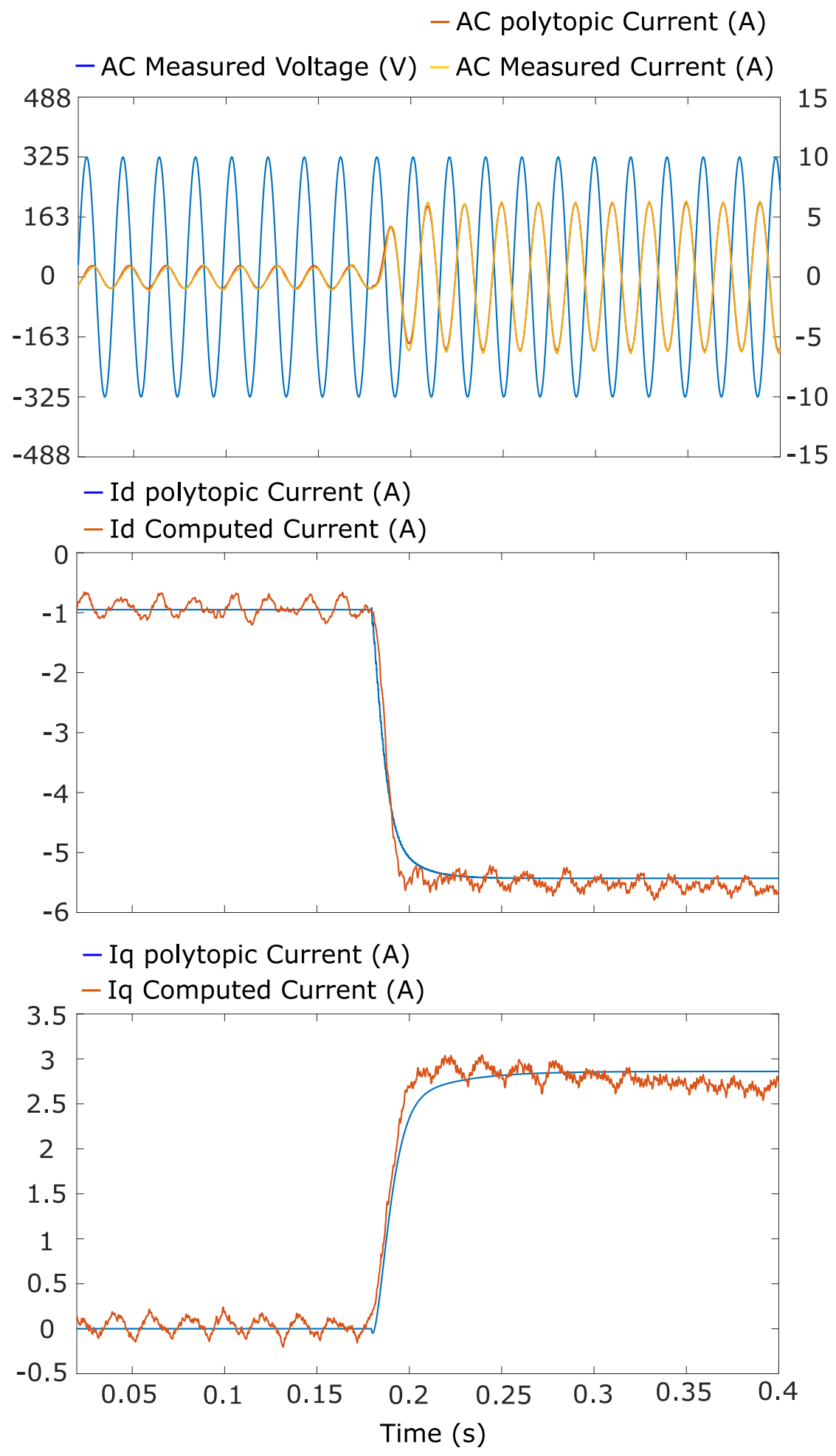

Figure 4.18 Results comparison between switching and polytopic black-box model method for $A C$ current response (upper) with $d-q$ components in a -6A step test in V2G mode 


\begin{tabular}{|c|c|c|c|}
\hline Tests & $\begin{array}{l}\text { Transfer } \\
\text { Functions }\end{array}$ & Iref Steps & Identified Transfer Functions \\
\hline \multirow{8}{*}{ Test 2} & \multirow{4}{*}{$H_{\text {dref }}(z)$} & $-3 \mathrm{~A} \rightarrow-4 \mathrm{~A}$ & $\frac{-0.7467 e-3 z+0.7527 e-3}{z^{2}-1.995 z+0.9954}$ \\
\hline & & $-4 \mathrm{~A} \rightarrow-5 \mathrm{~A}$ & $\frac{2.331 e-3 z^{6}-3.15 e-3 z^{5}-5.513 e-3 z^{4}+11.29 e-3 z^{3}-4.952 z^{2}}{z^{7}-4.279 z^{6}+8.85 z^{5}-12.14 z^{4}+12.13 z^{3}-8.821 z^{2}+4.253 z-0.9908}$ \\
\hline & & $-5 \mathrm{~A} \rightarrow-6 \mathrm{~A}$ & $\frac{4.896 e-5 z^{2}+5.853 e-5 z+1.24 e-5}{z^{3}-0.9653 z^{2}-z+0.9653}$ \\
\hline & & $-6 \mathrm{~A} \rightarrow-7 \mathrm{~A}$ & $\frac{-0.4211 e-3 z+0.59 e-3}{z^{2}-1.907 z+0.9074}$ \\
\hline & \multirow{4}{*}{$H_{\text {dref }}(z)$} & $-3 \mathrm{~A} \rightarrow-4 \mathrm{~A}$ & $\frac{0.1617 e-3 z-0.1635 e-3}{z^{2}-1.997 z+0.9968}$ \\
\hline & & $-4 \mathrm{~A} \rightarrow-5 \mathrm{~A}$ & $\frac{0.6846-3 z^{2}+2.335 e-6 z-0.6939 e-3}{z^{3}-0.9706 z^{2}-z+0.9706}$ \\
\hline & & $-5 \mathrm{~A} \rightarrow-6 \mathrm{~A}$ & $\frac{-0.2244 e-3 z-0.2317 e-3}{z^{2}+1.259 e-3 z-0.9987}$ \\
\hline & & $-6 \mathrm{~A} \rightarrow-7 \mathrm{~A}$ & $\frac{1.847 e-5 z-1.851 e-5}{z^{2}-1.999 z+0.9994}$ \\
\hline
\end{tabular}

Table 4.3 Identified transfer functions for V2G operation in z-domain.

\subsection{Experimental result in the setup 2 with Li-ion batteries}

In the experimental setup 2, two E-bike Lithium-ion batteries [100] were used. The batteries are of $48 \mathrm{~V}$ and 20.4Ah each connected in series. The maximum usable voltage that can reach in a charging mode is 50V, while the current until 5A. Both batteries are provided with a Battery Management System (BMS) by their provider.

Figure 4.19 illustrates the electric scheme and the setup image of the experiment. CINERGIA B2C30 equipment is operating as a current source and the DC output of the channel $U$, operates as an indicated point of connection between the 2 batteries in series. All experiments in this section will be applied through current set-point of channel $U$. This set of experiments is taking the current values of all threephases along with one phase of grid voltage and the DC battery current. Once more the experiments will contain V2G and G2V representations. All equipment used in this setup 2 is shown in the Table 4.4. 


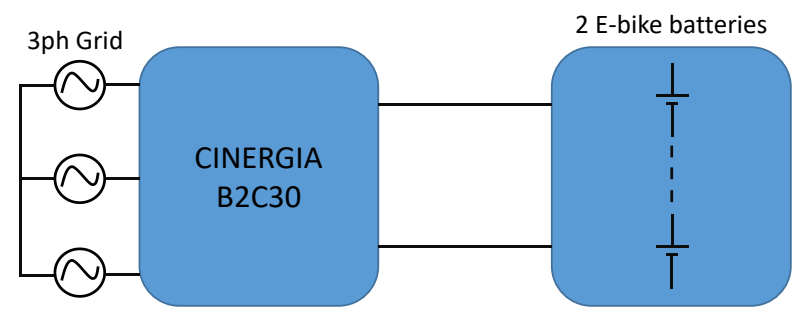

(a)

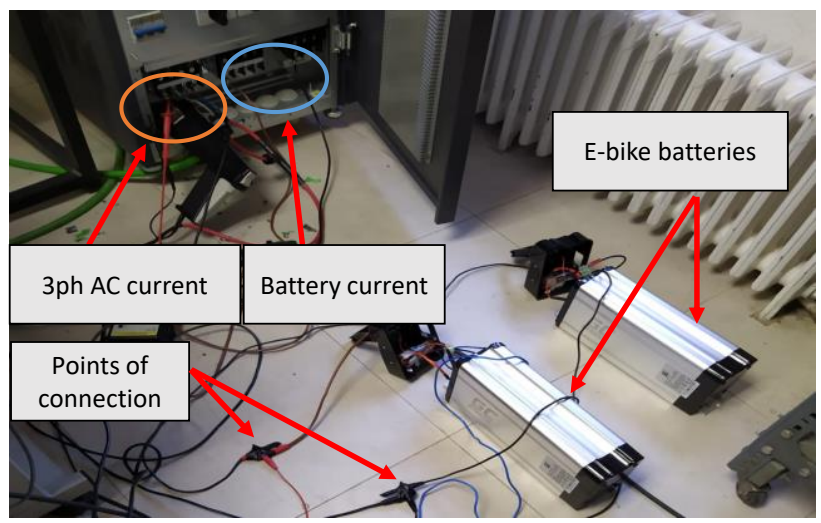

(b)

Figure 4.19 Experimental setup: (a) Electrical scheme, (b) Setup image

\begin{tabular}{|l|l|l|}
\hline Device & Model & Specifications \\
\hline Bidirectional battery charger & B2C30 & $\begin{array}{l}\text { DC part (independent mode): 750 Vmax } \\
\text { (using 400 V), +-30 A }\end{array}$ \\
\hline 2 E-BIKE Batteries & $\begin{array}{l}\text { GC E-BIKE Battery 48 V } \\
20.4 \mathrm{Ah}\end{array}$ & $\begin{array}{l}\text { Electric Bicycle Bike Silverfish Li-Ion } \\
\text { Battery }\end{array}$ \\
\hline Oscilloscope (2) & $\begin{array}{l}\text { ROHDE \& SCHWARZ RTE } \\
1104\end{array}$ & $1 \mathrm{GHz}, 5 \mathrm{GSa} / \mathrm{s}$ \\
\hline Differential (Voltage) probe & TESTEC TT -SI 9010 & $+-7 \mathrm{Kv}$ at1/1000 or +-700 V at 1/100 \\
\hline Current probes (6) & RT-ZC20B & $0.1 \mathrm{~V} / \mathrm{A}, 100 \mathrm{MHz}, 30 \mathrm{Arms}$ \\
\hline
\end{tabular}

Table 4.4 Main characteristics of the components and instruments in the experimental setup 


\subsubsection{Black-box model for experimental setup 2 of a battery charger with Lithium-Ion batteries}

In this setup 2, only the small-signal model has been tested, either in G2V or V2G operation. As in the previous experiments the $A C$ voltage is assumed remains constant and consequently, in the black-box model, only one input variable is considered, the battery current reference $I_{\text {ref }}$ (figure 4.20). At the same time, the model will have three outputs, the $d$ and q components of the AC current and the battery current. This implies that the small-signal black-box model requires to compute three transfer functions.

In the $\mathrm{G} 2 \mathrm{~V}$ mode, the selected operating point to obtain the model was $1 \mathrm{~A}$ for the reference in the battery current $I_{\text {ref }}$ and the step applied to obtain the model's transfer functions is $2 \mathrm{~A}$, from $1 \mathrm{~A}$ to $3 \mathrm{~A}$.

On the other hand, in the V2G mode, the selected operating point was $-11 \mathrm{~A}$ for the reference in the battery current $I_{\text {ref }}$ and the step applied to obtain the model's transfer functions was $3 A$, from - $11 A$ to $8 \mathrm{~A}$.

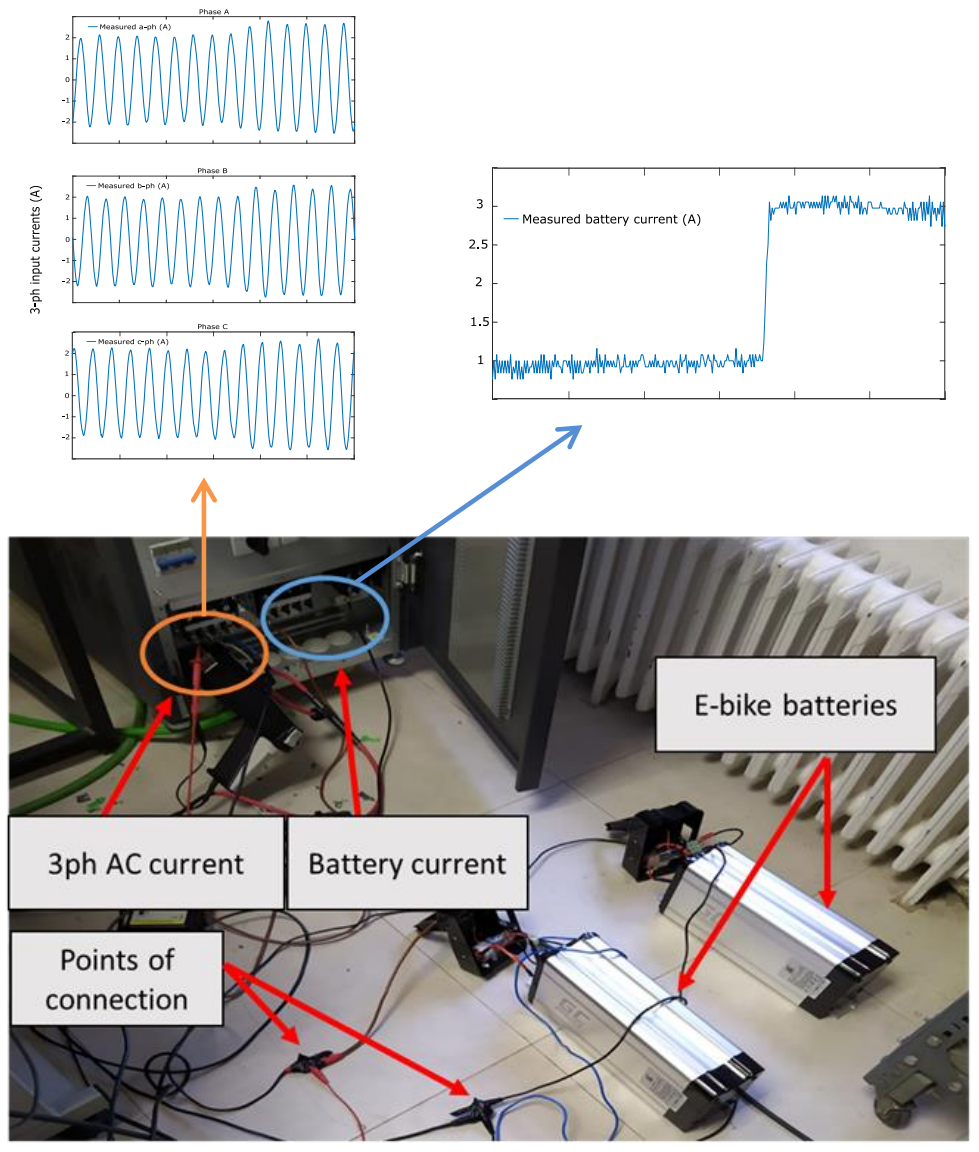

Figure 4.20 Experimental setup with Li-ion batteries and input/output points of data collection 


\subsubsection{Comparison of black-box model and experimental setup 2}

In this section, once the transfer functions of the small-signal models have obtained, the comparison between of the output variables estimated by the model and the actual measurement of the same variables are checked. As it was described in chapter 2 , the model needs to compute as many transfer functions as the number of outputs multiply by the number of inputs. In the experimental conditions of this setup 2, this means three transfer functions $H_{\text {dref }}$ and $H_{\text {qref }}$ for the $d$ and q components of the $A C$ current and $\mathrm{H}_{\text {batref }}$ for the battery current. All this transfer functions are related to perturbation in the reference for the battery current $I_{\text {ref. }}$. This transfer function for the selected operation points in G2V and V2G mode are listed in Table 4.5.

The first test (figure 4.21) is operating in G2V mode with all AC input current phases illustrated in the first three graphs followed by $d$-q components of the AC current in the fourth and the battery current in the last. Initially the operating point of the current is set at $1 \mathrm{~A}$ charging the battery and in a time of $\mathrm{t}=0.28 \mathrm{~s}$ a step of $2 \mathrm{~A}$ added to the reference current control reaching $3 \mathrm{~A}$. In orange colour it can be seen the blackbox model (except on the third graph with orange and purple) and in blue the measured results adapting with a good level of accuracy.

The second test (figure 4.22) it operates in V2G mode. Once more all AC input current phases are shown in the first three graphs followed by $d$-q components of the AC current in the fourth and the battery current in last. At start we can see the system to be in steady state with current at $-11 \mathrm{~A}$ and at $\mathrm{t}=0.28 \mathrm{~s}$ a $3 \mathrm{~A}$ is imposed taking the system at $-8 \mathrm{~A}$ in V2G mode of operation. Once more with orange colour the black-box model is observed (except on the third graph with orange and purple) while the measured results are in blue. As you can see again in this experiment, the dependence in the accuracy with magnitude of the perturbation. When the perturbation is becoming large enough ( $3 \mathrm{~A}$ in this case), the accuracy of the model is becoming worse. Within this environment of large-signal perturbations in the input variables, large-signal models, like the polytopic one, are needed. This means that the experimental results in setup 1 and setup 2 confirm the limited validity of linear models for power converters in general and for AC/DC converters. The identified functions created for the black-box modelling are listed in the table 4.5 in mathematical expressions.

\begin{tabular}{|c|c|c|}
\hline Transfer Functions & G2V & V2G \\
\hline$H_{\text {dref }}(s)$ & $\frac{126.8}{s+480.3}$ & $\frac{17.64}{s+115.3}$ \\
\hline$H_{\text {qref }}(s)$ & $\frac{1.338 e 6}{s^{2}+2740 s+2.124 e 7}$ & Neglected \\
\hline$H_{\text {batref }}(s)$ & $\frac{574.5}{s+574.5}$ & $\frac{7746}{s+7746}$ \\
\hline
\end{tabular}

Table 4.5 Identified transfer functions from the system level model experimental setup with Li-ion batteries 

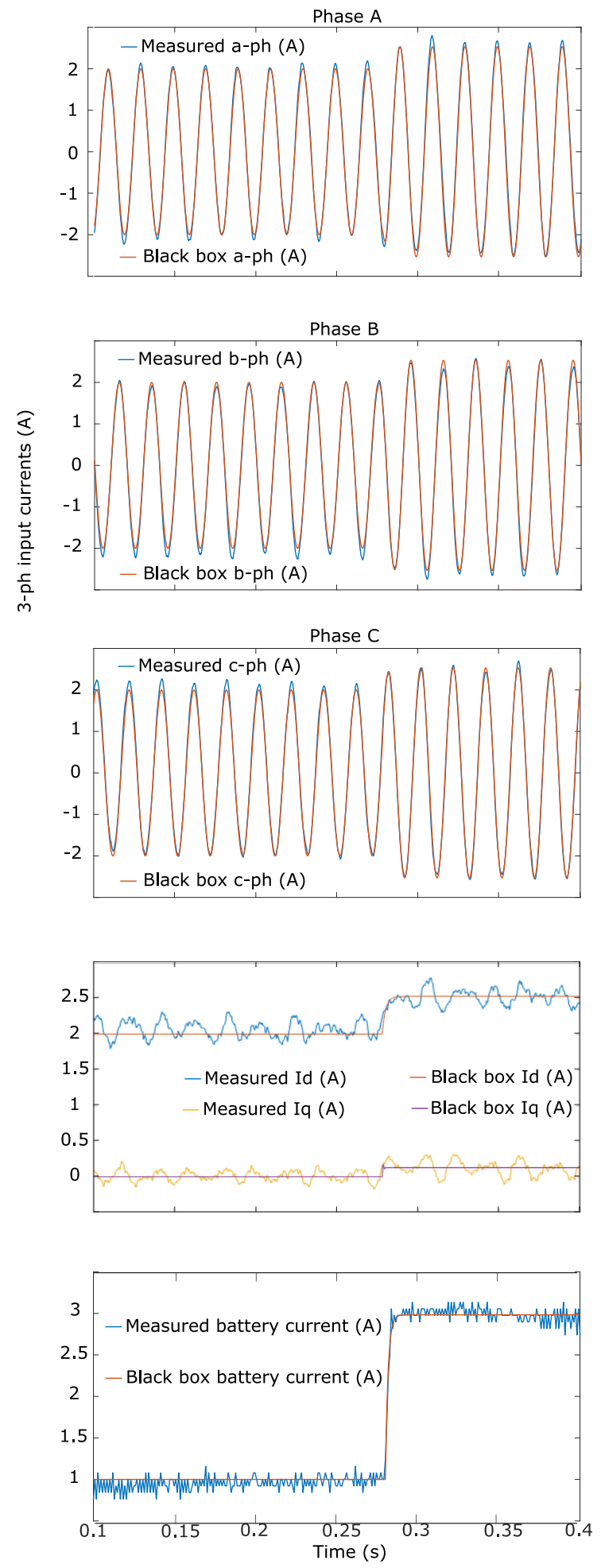

Figure 4.21 Results comparison between switching and black-box model for three AC current phases along with d-q components and the DC battery current for a $2 \mathrm{~A}$ in a G2V mode test 

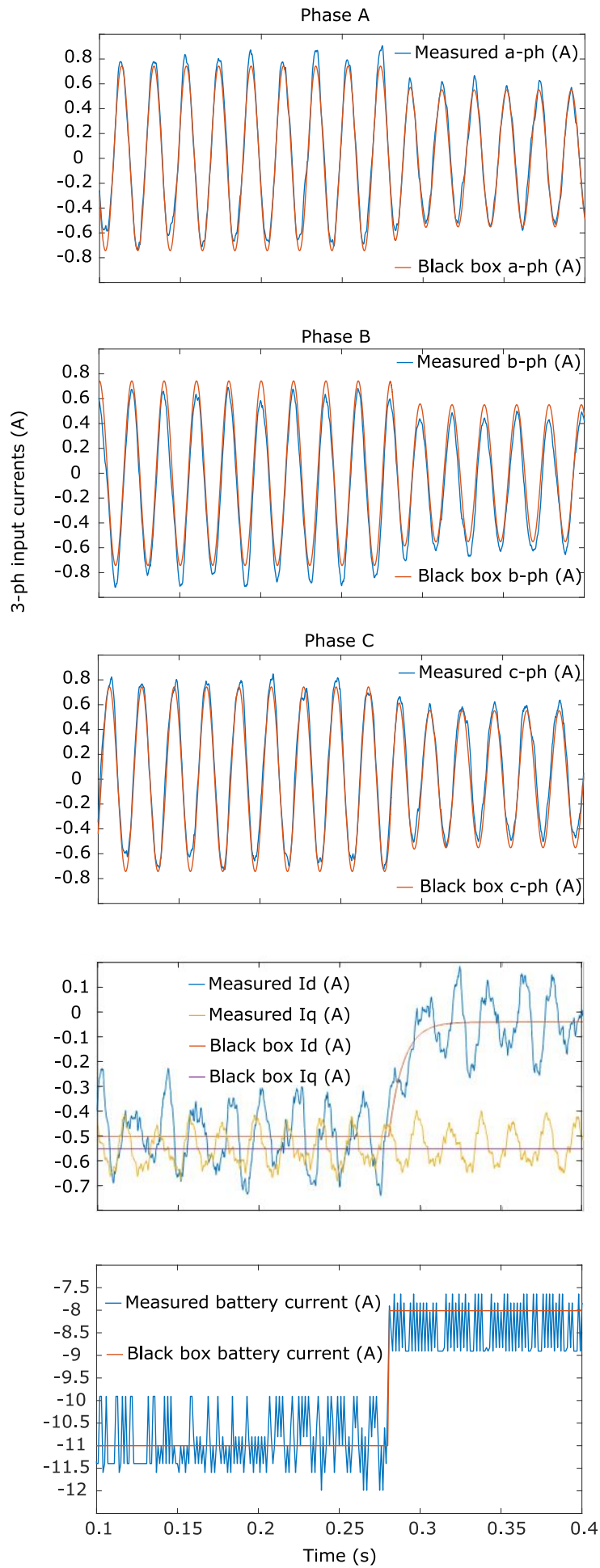

Figure 4.22 Results comparison between switching and black-box model for three AC current phases along with $d-q$ components and the DC battery current for a $+3 \mathrm{~A}$ step in a V2G mode test 



\section{CHAPTER}

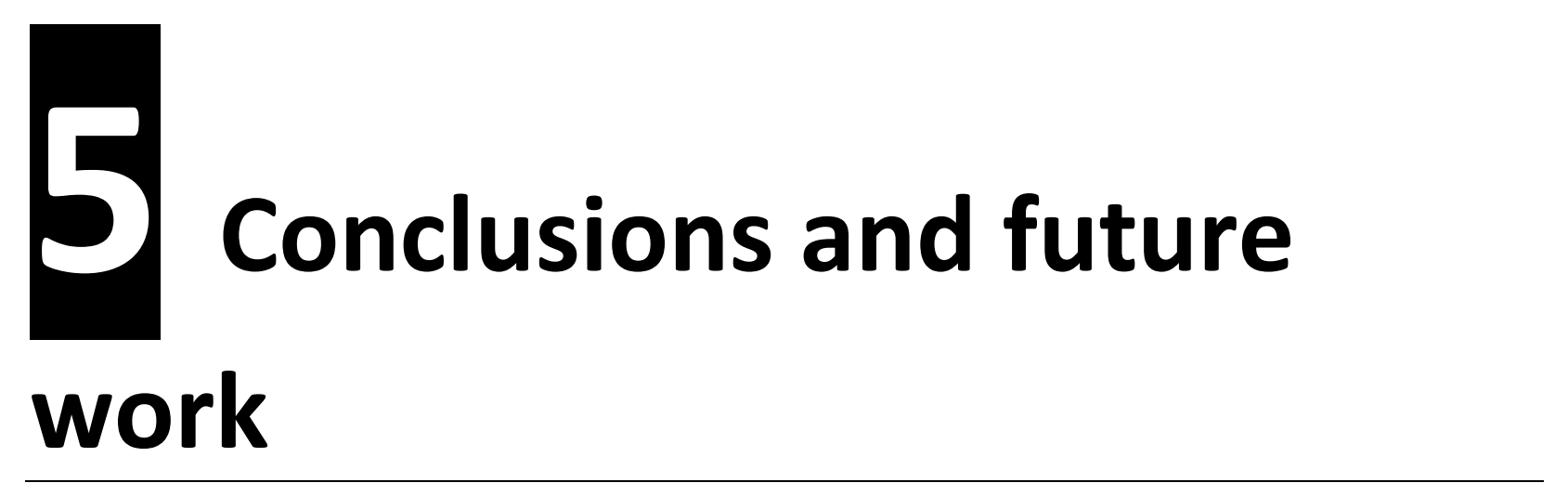

\subsection{Introduction}

The proliferation of electric vehicles is associated with a huge increase in the number of battery chargers, which will manage the exchange of energy between the electric vehicles and the grid. The integration of a huge number of AC/DC converters in the power distribution system, especially in islanded systems, can have a negative influence in its dynamic behaviour. Furthermore, the variety of commercial battery chargers, about which the information is very limited, make the system-level analysis of these architectures very difficult.

To cope this challenge, a possible solution is the use of black-box models, which can approximate the dynamic behaviour of power electronic converters by analysing the response of the output terminals of the devices to specific perturbances. The black-box approaches have been successfully applied to DC/DC converters and DC microgrids, and DC/AC converters in AC microgrids, however, the number of studies about AC/DC converters is very limited due to their complexity. This is the main contribution of this doctoral thesis

This work extends some of the black-box modelling techniques applied to DC/DC converters and threephase DC/AC converters to single-phase and three-phase AC/DC converters, in particular to electric vehicle AC/DC battery chargers. In a first approximation, the small-signal model is described, identifying the input and output variables, and transforming $A C$ signals into DC ones by means of the Park transformation. The small-signal condition limits the validity of the model to small perturbations of the input variables and the strong dependence of the steady-state operating point. This linear model has been both mathematically defined and represented with an electrical equivalent circuit. This is one of the main contributions of this work. 
This approach has been validated by means of simulations and experimental tests. It is particularly interesting the results from simulations using the virtual prototyping approach. In this approach the dynamic behaviour of the AC/DC converter simulated with a switching simulator is compared with the results produced by the black-box models. In practice, virtual prototyping is assuming that simulations from switching models like the ones obtained by PSIM are an accurate representation of the actual converter, and consequently these switching models can be used as the valid reference to check the accuracy of the black-box models.

The obtained results, both in virtual prototyping and in the experimental setup show how the black-box small-signal model is able to approximate the dynamic behaviour of single-phase and three-phase AC/DC battery chargers, subject to keep the small-signal limitation.

At the same this work tests the small-signal black-box model in case of large perturbations of the input variables showing a clear loss of accuracy to conclude the need to develop large-signal black-box in case of larger perturbations. The proposed approach is the polytopic model, already used in DC/DC and DC/AC converters, but no in two stages, single-phase and three-phase bidirectional AC/DC converters like battery chargers.

The polytopic model is based on the obtention of a group of local small-signal models in different operating points combined with nonlinear weighting functions operating as a single nonlinear structure. The weight of the different linear models depends on the instantaneous value of the input variables. These large-signal nonlinear models are one of the main contributions of this work.

The accurate selection of local models in combination with the correct selection of weighting functions is vital. Learning from previous work applied to modelling power converters, the Double sigmoid function is showed to be the most suitable weighting function for this application.

This large-signal, nonlinear polytopic approach have also tested by virtual prototyping and experimental results. In this case, virtual prototyping is essentially the same concept described for the small-signal model, using the switching model of the actual converter as a valid reference for the comparison. The experimental tests have been extended to both power flow directions, G2V and V2G operating modes.

The obtained results show a clear improvement of the results coming from the polytopic model when they are compared with the same results coming from the small-signal model in case of large perturbations in the input variables. These results validate the usage of the proposed large-signal blackbox models to represent the dynamic behaviour of single-phase and three-phase two-stage bidirectional battery chargers operating under large perturbations. 


\subsection{Conclusions}

Although most of the conclusions are implicit in the section 5.1, again, the main conclusions of this work are the following:

1. There are a continuous growth of battery chargers exchanging energy with the grid. The variety of commercial battery chargers, about which the information is very limited, make the systemlevel analysis of these architectures very difficult. A modelling strategy of these battery chargers to anticipate the dynamic behaviour with the grid is needed.

2. The main feature of the models should be the assessment of the impact in the interaction with the grid.

3. Black-box models, which can approximate the dynamic behaviour of power electronic converters by analysing the response of the output terminals of the devices to specific perturbances can be a good and promising solution. This is the focus of the thesis and the more general contribution

4. This work extends some of the black-box modelling techniques applied to DC/DC converters and three-phase DC/AC converters to single-phase and three-phase AC/DC converters. This is another general contribution

5. A small-signal, black-box model is proposed, identifying the input and output variables, and transforming AC signals into DC ones by means of the Park transformation. This is an specific and original contribution of this work.

6. This small-signal, black-box model has been validated by means of simulations and experimental tests. It is particularly interesting the results from simulations using the virtual prototyping concept. Virtual prototyping applied to battery chargers is another specific contribution of this thesis. This contribution is not focus in the modelling strategy, but it is focus on the procedure for its development.

7. The obtained results for the small-signal, black-box model, both in virtual prototyping and in the experimental setup show how the black-box small-signal model is able to approximate accurately the dynamic behaviour of single-phase and three-phase AC/DC battery chargers, limiting the value of perturbations to small values.

8. Experimental and virtual prototyping results for the small-signal black-box model, in case of large perturbations of the input variables, show a clear loss of accuracy to conclude the need to develop large-signal black-box 
9. For large-signal perturbations, a polytopic black-box model is proposed. This strategy follows previous works applied to model DC/DC and DC/AC power converters. This is an original and specific contribution of this thesis applied to in two stages, single-phase and three-phase bidirectional AC/DC converters like battery chargers.

10. This large-signal, polytopic, black-box model has been validated by means of simulations and experimental tests. The virtual prototyping concept have been also used in simulation. The experimental tests have been extended to both power flow directions, G2V and V2G operating modes.

11. The obtained results from the large-signal, polytopic, black-box model show a clear improvement of the results coming from the polytopic model when they are compared with the same results coming from the small-signal model in case of large perturbations in the input variables.

\subsubsection{Contributions}

\section{Articles}

- Antreas Naziris, Airan Frances, Rafael Asensi and Javier Uceda, "Black-Box Small-Signal Structure for Single-Phase and Three-Phase Electric Vehicle Battery Chargers," Published in: IEEE Access (Volume: 8), Date of Publication: 18 September 2020, Page(s): 170496 - 170506

\section{Conference papers}

- Antreas Naziris, Rafael Asensi, and Javier Uceda, "Black box modelling of a bidirectional battery charger for electric vehicles," in Proc. 7th Int. Conf. Renew. Energy Res. Appl. (ICRERA), Oct. 2018, pp. 469-473.

- Antreas Naziris, Galo Guarderas, Airan Frances, Rafael Asensi, and Javier Uceda, "Large-Signal Black-Box Modelling of Bidirectional Battery Charger for Electric Vehicles," in IEEE Applied Power Electronics Conference and Exposition (APEC), March 2019. 


\subsection{Future work}

In this section, some items for future work are suggested. When someone spends four/five years doing research around a topic, she/he always has the impression that many things are still pending. Let me suggest some of those thoughts, some of those pending matters, about the potential areas for future development. First, it is important to remark that the purpose of this research is the assessment of the impact to the grid of a huge number of battery chargers connected to it. This means that the objective of this research is not to help the charger's manufactures to optimize their designs. What is important is to foresee the AC current and the requested active and reactive power demanded or injected by the charger. Having this information precisely, the technical grid operator will be able to manage the demand and take measure to ensure the stability of operation.

1. The experimental setup needs to closer to the actual battery chargers. In this thesis a bidirectional, very flexible and high-power AC/DC power converter (CINERGIA B2C30), but sometimes this converter behaves something different from a commercial battery charger in its interaction with the grid.

2. In the small-signal models, some more tests need to be done, particularly to assess the practical limitation to small-signal models, in terns of the amplitude of the perturbation.

3. In the large-signal model, when more than one input variable like the AC voltage or the reference for the reactive current, the selection of the operating points needs to be investigated.

4. The weighting functions are the crucial part of the polytopic model. Some other proposals like the dynamic weighting functions needs to be investigated.

5. The models are obtained through tests, but the converters along the time may change due to many different reasons. Is it possible to identify the black-box model in real time?

6. What is the impact of faulty and distorted grids in the modelling strategy? At this moment, in case of unbalanced, faulty or distorted grids, the proposed modelling strategy doesn't work.

7. What is about stability issues in nonlinear systems? Large-signal nonlinear stability methods like Lyapunov criterium are much more complex than in linear system. 



\section{Bibliography}

[1] European Commission. Climate change, "Overview of EU policy, legislation and action to address climate change." [Online]. Retrieved from https://ec.europa.eu/info/energy-climate-changeenvironment/topics/climate-change_en

[2] Javatpoint, "Conventional sources of energy vs non-conventional sources of energy." [Online]. https://www.javatpoint.com/conventional-sources-of-energy-vs-non-conventional-sources-of-energy

[3] European Commission. Energy, Climate change, Environment. Climate Action. EU Action. Climate strategies \& targets. Progress made in cutting emissions, "Emissions monitoring \& reporting." [Online]. Retrieved from https://ec.europa.eu/clima/policies/strategies/progress/monitoring_en

[4] European Commission. "Energy, Climate Change, Environment." [Online]. Retrieved from https://ec.europa.eu/info/energy-climate-change-environment_en."

[5] TUDelft OpenCourseWare. "Electric Cars: Technology. 1.1.1 Lecture: Motor and Energy Storage." [Online]. Available in the online page https://ocw.tudelft.nl/course-lectures/electric-cars-technology$18 /$ ?course id $=29663$

[6] European Federation for Transport and Environment. "How vehicle taxes can accelerate electric car sales." February 2019 [Online]. Available in the online page https://www.transportenvironment.org/sites/te/files/publications/2019 02 How vehicle taxes can a ccelerate electric car final.pdf

[7] European Environment Agency. "Fiscal instruments favouring electric over conventional cars are greener." [Online]. Retrieved from https://www.eea.europa.eu/themes/transport/electricvehicles/taxes-and-incentives-promoting-electric

[8] GOV.UK. "Vehicle tax rates." [Online]. Retrieved from https://www.gov.uk/vehicle-tax-rate-tables

[9] WWF 2016 report (HK). "Climate Change and Financial Risk", [Online]. Retrieved from http://awsassets.wwfhk.panda.org/downloads/wwf_ev_report_sp_final.pdf

[10] 2degreesinstitute. "Comparing Fuel and Maintenance Costs of Electric and Gas-Powered Vehicles in Canada.", September 2018 [Online]. Available in the online page https://www.2degreesinstitute.org/reports/comparing fuel and maintenance costs of electric and gas powered vehicles in canada.pdf

[11] EVgo Services LLC. BEVS, PHEVS AND HEVS, "Which Electric Vehicle Do You Drive Types of Electric Vehicles." [Online]. Retrieved from https://www.evgo.com/why-evs/types-of-electric-vehicles/ 
[12] Department of Energy. Office of Energy Efficiency \& Renewable Energy. "Electric Vehicle Basics". [Online]. Retrieved from https://www.energy.gov/eere/electricvehicles/electric-vehicle-basics

[13] U.S. Department of Energy. Energy Efficiency \& Renewable Energy. Alternative Fuel Data Center. "How Do Hybrid Electric Cars Work?". [Online]. Retrieved from https://afdc.energy.gov/vehicles/how-dohybrid-electric-cars-

work\#: :text=Hybrid\%20electric\%20vehicles\%20are\%20powered,by\%20the\%20internal\%20combustion \%20engine

[14] TUDelft OpenCourseWare. "Electric Cars: Technology. 1.3.1 Lecture: Drivetrains of Conventional and (hybrid) Electric Vehicles." [Online]. Retrieved from https://ocw.tudelft.nl/course-lectures/electric-carstechnology-6/?course id=29663

[15] TUDelft OpenCourseWare. "Electric Cars: Technology. 3.1.2 Lecture Notes: Introduction to Charging Methods of EVs." [Online]. Retrieved from https://ocw.tudelft.nl/course-readings/electric-carstechnology-19/

[16] TUDelft OpenCourseWare. "Electric Cars: Technology. 3.2.1 Lecture: AC charging of EVs." [Online]. Retrieved from https://ocw.tudelft.nl/course-lectures/electric-cars-technology-3/?course id=29663

[17] TUDelft OpenCourseWare. "Electric Cars: Technology. 3.3.1 Lecture: DC charging of EVs." [Online]. Retrieved from https://ocw.tudelft.nl/course-lectures/3-3-1-lecture-dc-charging-evs/?course id=29663

[18] TUDelft OpenCourseWare. "Electric Cars: Technology. 3.4.1 Lecture: Smart charging and V2G." [Online]. Retrieved from https://ocw.tudelft.nl/course-lectures/electric-cars-technology$\underline{7 / \text { ?course } i d=29663}$

[19] Mcdavis A. Fasugba and Philip T. Krein, "Gaining vehicle-to-grid benefits with unidirectional electric and plug-in hybrid vehicle chargers," 2011 IEEE Vehicle Power and Propulsion Conference, DOI: 10.1109/VPPC.2011.6043207

[20] Komatsu Yasuhiro, Tur-Amgalan Amarsanaa, Yoshihiko Araki, Syed Abdul Kadir Zawawi and Takamura Keita, "Design of the unidirectional current type coreless DC brushless motor for electrical vehicle with low cost and high efficiency," Date Added to IEEE Xplore: 09 August 2010, Published in: SPEEDAM 2010, DOI: 10.1109/SPEEDAM.2010.5545147

[21] Vishnu Mahadeva lyer, Srinivas Gulur and Subhashish Bhattacharya, "Small-signal modeling and stability analysis of a bidirectional electric vehicle charger," 2017 IEEE 6th International Conference on Renewable Energy Research and Applications (ICRERA), 5-8 Nov. 2017. 
[22] Vishnu Mahadeva Iyer, Srinivas Gulur and Subhashish Bhattacharya, "Variable DC bus control for a bidirectional on-board electric vehicle charger," 2017 IEEE 6th International Conference on Renewable Energy Research and Applications (ICRERA), 5-8 Nov. 2017

[23] Union of concerned scientists. Science for a healthy planet and safer world [BLOG]. "Comparing Electric Vehicles: Hybrid vs. BEV vs. PHEV vs. FCEV." [Online]. Retrieved from https://blog.ucsusa.org/josh-goldman/comparing-electric-vehicles-hybrid-vs-bev-vs-phev-vs-fcev-411

[24] Fang Jian, Wang Yong, Luan Le and Li Shengnan, "Frequency domain harmonic model of electric vehicle charger using three-phase uncontrolled rectifier." 2016 China International Conference on Electricity Distribution (CICED), Date Added to IEEE Xplore: 26 September 2016, DOI: 10.1109/CICED.2016.7575912

[25] Wenhai Yang, Jingmin Wang, Zhanlong Zhang and Yajing Gao, "Simulation of electric vehicle charging station and harmonic treatment." 2012 International Conference on Systems and Informatics (ICSAI2012), Date Added to IEEE Xplore: 25 June 2012, DOI: 10.1109/ICSAI.2012.6223071

[26] Murat Yilmaz and Philip T. Krein, "Review of Battery Charger Topologies, Charging Power Levels, and Infrastructure for Plug-In Electric and Hybrid Vehicles." Published in: IEEE Transactions on Power Electronics (Volume: 28, Issue: 5, May 2013), Date of Publication: 23 August 2012, Ol: 10.1109/TPEL.2012.2212917

[27] Bhanu Teja Vankayalapati, Rajeev Singh and Vinod Kumar Bussa, "Two stage integrated on-board charger for EVs." Published in 2018 IEEE International Conference on Industrial Technology (ICIT). DOI: 10.1109/ICIT.2018.8352458

[28] Jun-Mo Kim, Jeong Lee, Tae-Ho Eom, Kyung Ryu, Min-Ho Shin and Chung-Yuen Won, "Modular EV Rapid Charger Design and Control Method." Published in: 2019 22nd International Conference on Electrical Machines and Systems (ICEMS). Date Added to IEEE Xplore: 05 December 2019, DOI: 10.1109/ICEMS.2019.8922320

[29] Vítor Monteiro, J. G. Pinto, Bruno Exposto, Luís F. C. Monteiro, Carlos Couto and João L. Afonso, "A novel concept of unidirectional bridgeless combined boost-buck converter for EV battery chargers." Published in: 2015 IEEE 24th International Symposium on Industrial Electronics (ISIE), DOI: 10.1109/ISIE.2015.7281467

[30] Huaibao Wang, Xiaoyu Jia, J. Li, X. Guo, B. Wang and Xiaoyu Wang, "New single-stage EV charger for V2H applications." Published in: 2016 IEEE 8th International Power Electronics and Motion Control Conference (IPEMC-ECCE Asia). DOI: 10.1109/IPEMC.2016.7512724 
[31] Tomokazu Mishima and Shoya Mitsui, "A Single-Stage High Frequency-link Modular Three-Phase SoftSwitching AC-DC Converter for EV Battery Charger." Published in: 2019 IEEE Energy Conversion Congress and Exposition (ECCE). DOI: 10.1109/ECCE.2019.8913110

[32] Byeongwoo Kim, Minjae Kim and Sewan Choi, "Single-stage electrolytic capacitor-less AC-DC converter with high frequency isolation for EV charger." Published in: 2016 IEEE 8th International Power Electronics and Motion Control Conference (IPEMC-ECCE Asia). DOI: 10.1109/IPEMC.2016.7512291

[33] Anant Kumar Singh, Rajagopal Prasanna and Kaushik Rajashekara, "Modelling and Control of Novel Bidirectional Single-Phase Single-Stage Isolated AC-DC Converter with PFC for Charging of Electric Vehicles." Published in: 2018 IEEE International Conference on Electro/Information Technology (EIT). DOI: 10.1109/EIT.2018.8500239

[34] Ali Tausif, Hoyoung Jung and Sewan Choi, "Single-stage isolated electrolytic capacitor-less ev onboard charger with power decoupling." Published in: CPSS Transactions on Power Electronics and Applications (Volume: 4, Issue: 1, March 2019). DOI: 10.24295/CPSSTPEA.2019.00004

[35] Minji Kim, Takongmo Marius, Gangwoo Lee, Jaesung Oh, Kyungjong Yoo, Eunsoo Kim and Ingab Hwang, "A Single-Stage Three-Level AC/DC Converter for Wireless Power Transfer." Published in: 2019 IEEE Applied Power Electronics Conference and Exposition (APEC). DOI: 10.1109/APEC.2019.8722248

[36] Hulong Zeng, Xiaorui Wang and Fang Zheng Peng, "High Power Density Z-Source Resonant Wireless Charger With Line Frequency Sinusoidal Charging." Published in: IEEE Transactions on Power Electronics (Volume: 33, Issue: 12, Dec. 2018). DOI: 10.1109/TPEL.2018.2806919

[37] Alberto Delgado, Jesús A. Oliver, José A. Cobos, Jorge Rodriguez and Alejandro Jiménez, "Optimized Design for Wireless Coil for Electric Vehicles Based on The Use of Magnetic Nano-articles." Published in: 2019 IEEE Applied Power Electronics Conference and Exposition (APEC). DOI: 10.1109/APEC.2019.8721998

[38] B.Lunz and D.U.Sauer, "Electric road vehicle battery charging systems and infrastructure." Published in: Advances in Battery Technologies for Electric Vehicles, 2015., Pages 445-467. Available online 29 May 2015.

[39] Circuit Digest. "Electric Vehicle On-board Chargers and Charging Stations" by Aswinth Raj. [Online]. Retrieved from https://circuitdigest.com/article/electric-vehicle-on-board-chargers-and-chargingstations

[40] Texas Instruments. "Taking charge of electric vehicles - both in the vehicle and on the grid" by Xun Gong and Jayanth Rangaraju. [Online]. Retrieved from http://www.ti.com/lit/wp/szzy007/szzy007.pdf 
[41] Eaton Corporation. "On-Board Vs. Off Board Charging" by John Wirtz [Online]. Retrieved from https://s3.amazonaws.com/automotiveworld/slides/amusa2012/John+Wirtz+-+Eaton.pdf

[42] Vitor Monteiro, Joao C. Ferreira, Andres A. Nogueiras Melendez, Jose A. Afonso, Carlos Couto and Joao L. Afonso, "Experimental Validation of a Bidirectional Three-Level dc-dc Converter for On-Board or Off-Board EV Battery Chargers." Published in: IECON 2019 - 45th Annual Conference of the IEEE Industrial Electronics Society. DOI: 10.1109/IECON.2019.8927763

[43] Andersen-EV. "WHAT POWER DO I NEED TO CHARGE MY ELECTRIC VEHICLE?” [Online]. Retrieved from https://andersen-ev.com/charging-academy/what-power-do-i-need-to-charge-my-electric-vehicle/

[44] Kang Miao Tan, Vigna K. Ramachandaramurthy and Jia Ying Yong, "Bidirectional battery charger for electric vehicle." Published in: 2014 IEEE Innovative Smart Grid Technologies - Asia (ISGT ASIA). DOI: 10.1109/ISGT-Asia.2014.6873826

[45] Wallbox. "Why Bidirectional Charging is The Next Big Thing for EV Owners." [Online]. Retrieved from https://wallbox.com/en us/bidirectional-ev-charger

[46] Manuele Bertoluzzo, Nima Zabihi and Giuseppe Buja, "Overview on battery chargers for plug-in electric vehicles." Published in: 2012 15th International Power Electronics and Motion Control Conference (EPE/PEMC). DOI: 10.1109/EPEPEMC.2012.6397461

[47] T. Jalakas, I. Roasto and D. Vinnikov, "Analysis of battery charger topologies for an electric vehicle." Published in: 2012 13th Biennial Baltic Electronics Conference. Date Added to IEEE Xplore: 11 December 2012. DOI: 10.1109/BEC.2012.6376857

[48] R.D. Middlebrook, S. Cuk, "A general unified approach to modeling switching converter power stages", Published in: 1976 IEEE Power Electronics Specialists Conference. ZEEE PESC 76 Records, pp. 1834.

[49] W.M. Polivka, P.R.K. Chetty and R. D. Middlebrook, "State-Space Average Modelling of Converters with Parasitics and Storage-Time Modulation", Published in: 1980 IEEE Power Electronics Specialists Conference. DOI: 10.1109/PESC.1980.7089440.

[50] B. Johansson and M. Lenells, "Possibilities of obtaining small-signal models of DC-to-DC power converters by means of system identification", Published in: Published in: INTELEC. Twenty-Second International Telecommunications Energy Conference (Cat. No.00CH37131).DOI: 10.1109/INTLEC.2000.884229

[51] Silicon Republic. "What are the benefits of white-box models in machine learning?" by Ellen Tannam [Online]. Retrieved from https://www.siliconrepublic.com/enterprise/white-box-machine-learning 
[52] Software Testing Class. "Difference between Black Box Testing and White Box Testing" [Online]. Retrieved from https://www.softwaretestingclass.com/difference-between-black-box-testing-andwhite-box-testing/

[53] Christopher Henard, Mike Papadakis, Mark Harman, Yue Jia and Yves Le Traon, "Comparing WhiteBox and Black-Box Test Prioritization." Published in: 2016 IEEE/ACM 38th International Conference on Software Engineering (ICSE). DOI: 10.1145/2884781.2884791

[54] Francisco Cruz Naranjo and Gonzalo Acuna Leiva, "Indirect Training with Error Backpropagation in Gray-Box Neural Model: Application to a Chemical Process." Published in: 2010 XXIX International Conference of the Chilean Computer Science Society. Date Added to IEEE Xplore: 15 April 2011. DOI: 10.1109/SCCC.2010.41

[55] Octavio Loyola-González, "Black-Box vs. White-Box: Understanding Their Advantages and Weaknesses From a Practical Point of View." Published in: IEEE Access (Volume: 7). DOI: 10.1109/ACCESS.2019.2949286

[56] Javatpoint. "Black Box Testing vs. White Box Testing vs. Grey Box Testing." [Online]. Retrieved from https://www.javatpoint.com/black-box-testing-vs-white-box-testing-vs-grey-box-testing

[57] Francés Roger, Airán; Asensi Orosa, Rafael and Uceda Antolín, Javier (2019). "Blackbox Polytopic Model With Dynamic Weighting Functions for DC-DC Converters." IEEE Access, v. 7; pp. 160263-160273. ISSN 2169-3536. https://doi.org/10.1109/ACCESS.2019.2950983.

[58] Guarderas Burbano, Galo Fernando; Francés Roger, Airán; Ramírez Prieto, Dionisio; Asensi Orosa, Rafael and Uceda Antolín, Javier (2019). "Blackbox Large-Signal Modeling of Grid-Connected DC-AC Electronic Power Converters." Energies, v.12 (n.6); pp.1-22. ISSN19961073 https://doi.org/10.3390/en12060989

[59] Antreas Naziris, Airan Frances, Rafael Asensi and Javier Uceda, "Black-Box Small-Signal Structure for Single-Phase and Three-Phase Electric Vehicle Battery Chargers," Published in: IEEE Access (Volume: 8), Date of Publication: 18 September 2020, Page(s): 170496 - 170506

[60] M. Veerachary, Anmol Ratna Saxena, "G-Parameter Based Stability Analysis of DC-DC Power Electronic System," 2008 Joint International Conference on Power System Technology and IEEE Power India Conference, 09 January 2009.

[61] A. Francés, R. Asensi, Ó. García, R. Prieto and J. Uceda, "Modeling Electronic Power Converters in Smart DC Microgrids-An Overview," in IEEE Transactions on Smart Grid, vol. 9, no. 6, pp. 6274-6287, Nov. 2018. 
[62] A. Francés-Roger, A. Anvari-Moghaddam, E. Rodríguez-Díaz, J. C. Vasquez, J. M. Guerrero and J. Uceda, "Dynamic Assessment of COTS Converters-Based DC Integrated Power Systems in Electric Ships," in IEEE Transactions on Industrial Informatics, vol. 14, no. 12, pp. 5518-5529, Dec. 2018.

[63] O.P. Mandhana, R.G. Hoft, "Two port characterization of DC to DC resonant converters," Fifth Annual Proceedings on Applied Power Electronics Conference and Exposition, 06 August 2002.

[64] Dushan Boroyevich, Rolando Burgos, Luis Arnedo, Fei Wang, "Synthesis and Integration of Future Electronic Power Distribution Systems," 2007 Power Conversion Conference - Nagoya, 18 June 2007.

[65] Hao Liu, Yi Tang, Yu Feng, Xinghe Ma, “A Power Quality Disturbance Classification 423 Method Based on Park Transform and Clarke Transform Analysis," 2008 3rd 424 International Conference on Innovative Computing Information and Control, 22 August 4252008.

[66] L. Ljung, "System Identification: Theory for the user, Second Edition", Prentice-Hall, N.J., 1999

[67] Subhransu Padhee, Umesh Chandra Pati and Kamalakanta Mahapatra, "Modelling switched mode DC-DC converter using system identification techniques: A review," Published in: 2016 IEEE Students' Conference on Electrical, Electronics and Computer Science (SCEECS).DOI: 10.1109/SCEECS.2016.7509303

[68] N. Patcharaprakiti, K. Kirtikara, D. Chenvidhya, V. Monyakul and B. Muenpinij, "Modeling of Single Phase Inverter of Photovoltaic System Using System Identification," Published in: 2010 Second International Conference on Computer and Network Technology. DOI: 10.1109/ICCNT.2010.120

[69] Luis Arnedo, Rolando Burgos, Dushan Boroyevich and Fred Wang, "System-Level Black-Box Dc-to-Dc Converter Models," Published in: 2009 Twenty-Fourth Annual IEEE Applied Power Electronics Conference and Exposition. DOI: 10.1109/APEC.2009.4802861

[70] MathWork help center, "What are Hammerstein-Wiener Models?" Retrieved from https://www.mathworks.com/help/ident/ug/what-are-hammerstein-wiener-

models.htm|\#: :text=Hammerstein\%2DWiener\%20models\%20describe\%20dynamic,dynamic\%20compo nent\%20of\%20the 220 model.\&text=B\%2FF\%20is\%20a\%20linear,F)w(t).

[71] MathWork help center, "Identifying Hammerstein-Wiener Models" Retrieved from https://www.mathworks.com/help/ident/ug/identifying-hammerstein-wiener-models.html

[72] J. A. Oliver;R. Prieto;J. A. Cobos;O. Garcia;P. Alou, "Hybrid Wiener-Hammerstein Structure for GreyBox Modeling of DC-DC Converters," Published in: 2009 Twenty-Fourth Annual IEEE Applied Power Electronics Conference and Exposition. DOI: 10.1109/APEC.2009.4802669.

[73] J.Sjöberg, H.Hjalmarsson and L.Ljung, "Neural Networks in System Identification," Published in Scinece Direct. IFAC Proceedings Volumes Volume 27, Issue 8, July 1994, Pages 359-382 
[74] Oludare Isaac Abiodun, Aman Jantan, Oludare E Omolara, Kemi Dada, Nachaat Mohamed, and Humaira Arshad, "State-of-the-art in artificial neural network applications: A survey," Published by Elsevier, November 2018, DOI: 10.1016/j.heliyon.2018.e00938

[75] A. Francés, R. Asensi, O. García, R. Prieto, and J. Uceda, "The performance of polytopic models in smart dc microgrids," in Proc. IEEE Energy Conversion Congr. Exposition, Sept 2016, pp. 1-8.

[76] Kasra Arnavaz and Seyyed Kamaleddin Yadavar Nikravesh, "A Stabilizing Model Predictive Control for Nonlinear Fractional Order Systems with Polytopic Model," Published in: 2017 5th RSI International Conference on Robotics and Mechatronics (ICRoM). Date Added to IEEE Xplore: 17 September 2018. DOI: 10.1109/ICRoM.2017.8466133.

[77] Joan Rocabert, Alvaro Luna, Frede Blaabjerg and Pedro Rodríguez, "Control of Power Converters in AC Microgrids." Published in: IEEE Transactions on Power Electronics (Volume: 27, Issue: 11, Nov. 2012). DOI: 10.1109/TPEL.2012.2199334

[78] Seong-Chon Choi, Doo-Young Jung, Ji-Hwan Kim, Chung-Yuen Won and Yong-Chae Jung, "Control of multi-functional rapid charger for electric vehicle." Published in: 2013 IEEE International Symposium on Industrial Electronics. DOI: 10.1109/ISIE.2013.6563775

[79] Nabil Akel, Majid Pahlevaninezhad and Praveen Jain, "A D-Q rotating frame reactive power controller for single-phase bi-directional converters." Published in: 2014 IEEE 36th International Telecommunications Energy Conference (INTELEC). DOI: 10.1109/INTLEC.2014.6972121

[80] Rawad Zgheib, Kamal Al-Haddad and Innocent Kamwa, "V2G, G2V and active filter operation of a bidirectional battery charger for electric vehicles." Published in: 2016 IEEE International Conference on Industrial Technology (ICIT). DOI: 10.1109/ICIT.2016.7474935

[81] B. Singh, A. Chandra, and K. Al-Haddad, Power quality- Problems and mitigation techniques. United Kingdom: Wiley, 2015.

[82] Sonia Pinto, J. Fernando Silva, Filipe Silva and Pedro Frade, "Design of a Virtual Lab to Evaluate and Mitigate Power Quality Problems Introduced by Microgeneration." Open access peer-reviewed chapter. Submitted: October 26th 2010Reviewed: May 1st 2011Published: November 21st 2011 DOI: 10.5772/18185 [Online]. Retrieved from https://www.intechopen.com/books/electrical-generation-anddistribution-systems-and-power-quality-disturbances/design-of-a-virtual-lab-to-evaluate-and-mitigatepower-quality-problems-introduced-by-microgeneratio

[83] Ricardo G. Gago, Sónia F. Pinto and José F. Silva, "G2V and V2G electric vehicle charger for smart grids." Published in: 2016 IEEE International Smart Cities Conference (ISC2). DOI: 10.1109/ISC2.2016.7580786 
[84] Texas Instruments, "Design Guide: TIDA-010054 Bi-Directional, Dual Active Bridge Reference Design for Level 3 Electric Vehicle Charging Stations." [Online]. Available in the online page: http://www.ti.com/lit/ug/tidues0/tidues0.pdf?\&ts=1589466072662

[85] PES-Publications. "Modeling and Optimization of Bidirectional Dual Active Bridge DC-DC Converter Topologies." by FLORIAN KRISMER. Doctoral dissertation [Online]. Retrieved form https://www.pespublications.ee.ethz.ch/uploads/tx ethpublications/Krismer 20110317 Modeling and Optimization of Bidirectional Dual Active Bridge DC-DC Converter Topologies.pdf

[86] Alberto Rodríguez, Aitor Vázquez, Diego G. Lamar, Marta M. Hernando and Javier Sebastián, "Different Purpose Design Strategies and Techniques to Improve the Performance of a Dual Active Bridge With Phase-Shift Control." Published in: IEEE Transactions on Power Electronics (Volume: 30, Issue: 2, Feb. 2015). DOI: 10.1109/TPEL.2014.2309853

[87] Ari Hentunen, Teemu Lehmuspelto and Jussi Suomela, "Time-Domain Parameter Extraction Method for Thévenin-Equivalent Circuit Battery Models." Published in: IEEE Transactions on Energy Conversion (Volume: 29, Issue: 3, Sept. 2014). DOI: 10.1109/TEC.2014.2318205

[88] B. Schweighofer, K.M. Raab and G. Brasseur, "Modeling of high power automotive batteries by the use of an automated test system." Published in: IEEE Transactions on Instrumentation and Measurement (Volume: 52, Issue: 4, Aug. 2003). DOI: 10.1109/TIM.2003.814827

[89] Min Chen and G.A. Rincon-Mora, "Accurate electrical battery model capable of predicting runtime and I-V performance." Published in: IEEE Transactions on Energy Conversion (Volume: 21, Issue: 2, June 2006). DOI: 10.1109/TEC.2006.874229

[90] Radha Kushwaha and Bhim Singh, "An EV battery charger based on PFC Sheppard Taylor Converter." Published in: 2016 National Power Systems Conference (NPSC). DOI: 10.1109/NPSC.2016.7858944

[91] Lumen learning courses. "Energy Stored in Capacitors." [Online]. Available in the online page https://courses.lumenlearning.com/physics/chapter/19-7-energy-stored-in-capacitors/

[92] Nima Tashakor, Ebrahim Farjah, Seyed Reza Khayam Hosseini and Teymoor Ghanbari, "A high performance bi-directional AC-DC converter for charge equalization." Published in: 2016 7th Power Electronics and Drive Systems Technologies Conference (PEDSTC). DOI: 10.1109/PEDSTC.2016.7556866

[93] Weixiang Shen, Thanh Tu Vo and Ajay Kapoor, "Charging algorithms of lithium-ion batteries: An overview." Published in: 2012 7th IEEE Conference on Industrial Electronics and Applications (ICIEA). DOI: 10.1109/ICIEA.2012.6360973 
[94] Sandra Zeljkovic, Radovan Vuletic, Andreas Miller and Alann Denais, "Control of SiC-based dual active bridge in high power three phase on-board charger of EVs." Published in: 2015 International Conference on Electrical Systems for Aircraft, Railway, Ship Propulsion and Road Vehicles (ESARS). DOI: 10.1109/ESARS.2015.7101454

[95] R.C. Cope and Y. Podrazhansky, "The art of battery charging." Published in: Fourteenth Annual Battery Conference on Applications and Advances. Proceedings of the Conference (Cat. No.99TH8371). DOI: 10.1109/BCAA.1999.795996

[96] D. Vinko, I. Biondić and D. Bilandžija, "Soft-Start Circuit in Wireless Power Transmission System." Published in: 2019 42nd International Convention on Information and Communication Technology, Electronics and Microelectronics (MIPRO). DOI: 10.23919/MIPRO.2019.8756886

[97] CINERGIA, Regenerative Power Electronics Solutions. B2C+ v6.1 Sept2019. [Online]. Available: https://www.cinergia.coop/wpcontent/uploads/2020/01/B2C-v6.1.pdf

[98] Valuetronics. DHP400-37 Sorensen $14.8 \mathrm{~kW}, 400 \mathrm{~V}, 37 \mathrm{~A}$ DC Power Supply. [Online]. Available: https://www.valuetronics.com/product/dhp400-37-sorensen-dc-power-supply-used

[99] Chroma Systems Solutions. High Power DC Electronic Load - 63200. [Online]. Available: https://www.chromausa.com/product/high-power-dc-electronic-load63200/\#: :text=The\%20Chroma\%20Electronic\%20Loads\%2063200,\%2C\%20batteries\%2C\%20and\%20fue \%20cells.

[100] Amazon.co.uk. GC E-BIKE Battery 48V 18Ah Li-Ion Silverfish with Charger Electric Bicycle. [Online]. Available:https://www.amazon.co.uk/Battery-Silverfish-Charger-Electric-Bicycle-

Silver/dp/B086W7MF8K 